\title{
Identification of Causality in Genetics and Neuroscience
}

\author{
Adèle Helena Ribeiro
}

\author{
DisSERTATION \\ PRESENTED TO THE \\ Institute OF MATHEMATICS AND STATISTICS \\ of THE UNIVERSITY OF SÃo PAUlo \\ IN PARTIAL FULFILLMENT OF THE REQUIREMENTS \\ FOR THE DEGREE OF \\ DOCTOR OF PHILOSOPHY \\ Program: Computer Science \\ Advisor: Prof. Dr. André Fujita \\ Co-Advisor: Prof. Dr. Júlia Maria Pavan Soler
}

The author acknowledges financial support provided by

CAPES Computational Biology grant no. 51/2013 and by

PDSE/CAPES process no. 88881.132356/2016-01

São Paulo, November 2018 


\section{Identification of Causality in Genetics and Neuroscience}

This is the corrected version of the $\mathrm{Ph}$.D. dissertation by Adèle Helena Ribeiro, as suggested by the committee members during the defense of the original document, on November 28, 2018.

Dissertation committee members: Prof. Dr. André Fujita - IME - USP

Profa. Dra. Julia Maria Pavan Soler - IME - USP Prof. Dr. Benilton de Sá Carvalho - IMECC - UNICAMP Prof. Dr. Luiz Antonio Baccala - EPUSP - USP Prof. Dr. Koichi Sameshima - FM - USP 


\section{Acknowledgments}

First, I thank my advisor Prof. André Fujita for all his support. He always provided me with many opportunities to grow as a researcher, including participation in conferences and collaboration with other researchers. I am very grateful for all his help in getting the research internship position at Princeton University and for all advice on my academic career.

I thank my co-advisor Prof. Júlia Maria Pavan Soler, who is an example of excellence as advisor, researcher, teacher, and friend. Her passion and dedication for science always inspired me. I also thank Julia's family for all the funny and inspiring moments.

Besides my advisor and co-advisor, I would like to thank the rest of the committee members, Prof. Luiz Antonio Baccala, Prof. Koichi Sameshima, and Prof. Benilton de Sá Carvalho, for their encouragement, recommendations, and time dedicated to review my dissertation. I also want to express my sincere gratitude to Prof. Guilherme J. M. Rosa for his valuable suggestions and discussions about my Ph.D dissertation.

I also would like to thank Prof. Daniel Y. Takahashi and Prof. Asif A. Ghazanfar for supervising me and supporting me during my research internship at Princeton University. They helped me a lot with everything I needed to develop my academic work as well as to make new friends and establish new collaborations.

I also thank Prof. José Krieger from InCor, the Heart Institute, for the academic opportunities and for making available to me the databases analyzed in this Ph.D. dissertation, particularly the Baependi Heart Study data.

I am deeply grateful to my beloved husband Max for all his love, care, and patience, even in stressful times, and for always supporting me to pursue my academic career.

I thank Prof. Roberto Hirata Jr., who supported me and encouraged me since I was an undergraduate student and always gave me good advice. I also thank all the students and professors who collaborate with the eScience lab, particularly the admins.

I present my special thanks to my parents José Diógenes Ribeiro and Vilma Helena da Silva for always taking care of me, for supporting me whenever I needed, and for understanding all the choices I made. I also thank my husband's family, Horst Reinhold Jahnke, Andrea Duarte Matias, and Gilda Timóteo Leite, for supporting us in the past few years.

I would like to express my deepest gratitude to Prof. Paulo Domingos Cordaro for being a great friend and for making our lunches much more pleasant.

I am deeply grateful to all my friends, especially Luciana, Maciel, Ana Ciconelle, Francisco, Ronaldo, Gregorio, Ana Carolina, Gabriel, Luis, Antonio and Nicholas, who have 
supported me throughout these years with their continuous encouragement and made my days so much better.

Last but not least, I would like to thank the Institute of Mathematics and Statistics of the University of São Paulo for the infrastructure and all professors, who were always available for helping me with mathematics, statistics, and computer science. 


\section{Resumo}

Ribeiro, Adèle. H. Identificação de Causalidade em Genética e Neurociência. 2018. Tese de Doutorado - Instituto de Matemática e Estatística, Universidade de São Paulo, São Paulo.

Inferência causal pode nos ajudar a compreender melhor as relações de dependência direta entre variáveis e, assim, a identificar fatores de riscos de doenças. Em Genética, a análise de dados agrupados em famílias permite investigar influências genéticas e ambientais nas relações entre as variáveis. Neste trabalho, nós propomos métodos para aprender, a partir de dados Gaussianos agrupados em famílias, o mais provável modelo gráfico probabilístico (dirigido ou não dirigido) e também sua decomposição em dois componentes: genético e ambiental. Os métodos foram avaliados por simulações e aplicados tanto aos dados simulados do Genetic Analysis Workshop 13, que imitam características dos dados do Framingham Heart Study, como aos dados da síndrome metabólica do estudo Corações de Baependi. Em Neurociência, um desafio consiste em identificar interações entre redes funcionais cerebrais - grafos. Nós propomos um método que identifica causalidade de Granger entre grafos e, por meio de simulações, mostramos que o método tem alto poder estatístico. Além disso, mostramos sua utilidade por meio de duas aplicações: 1) identificação de causalidade de Granger entre as redes cerebrais de dois músicos enquanto tocam um dueto de violino e 2) identificação de conectividade diferencial do hemisfério cerebral direito para o esquerdo em indivíduos autistas.

Palavras-chave: Modelos gráficos probabilísticos, Aprendizagem de estrutura, Modelo misto poligênico, Causalidade de Granger, Redes funcionais cerebrais. 


\section{Abstract}

Ribeiro, Adèle. H. Identification of Causality in Genetics and Neuroscience. 2018. Ph.D. dissertation - Institute of Mathematics and Statistics, University of São Paulo, São Paulo.

Causal inference may help us to understand the underlying mechanisms and the risk factors of diseases. In Genetics, it is crucial to understand how the connectivity among variables is influenced by genetic and environmental factors. Family data have proven to be useful in elucidating genetic and environmental influences, however, few existing approaches are able of addressing structure learning of probabilistic graphical models (PGMs) and family data analysis jointly. We propose methodologies for learning, from observational Gaussian family data, the most likely PGM and its decomposition into genetic and environmental components. They were evaluated by a simulation study and applied to the Genetic Analysis Workshop 13 simulated data, which mimic the real Framingham Heart Study data, and to the metabolic syndrome phenotypes from the Baependi Heart Study. In neuroscience, one challenge consists in identifying interactions between functional brain networks (FBNs) - graphs. We propose a method to identify Granger causality among FBNs. We show the statistical power of the proposed method by simulations and its usefulness by two applications: the identification of Granger causality between the FBNs of two musicians playing a violin duo, and the identification of a differential connectivity from the right to the left brain hemispheres of autistic subjects.

Keywords: Probabilistic graphical models, Structure learning, Polygenic mixed model, Granger causality, Functional brain networks. 


\section{Contents}

1 Introduction $\quad 1$

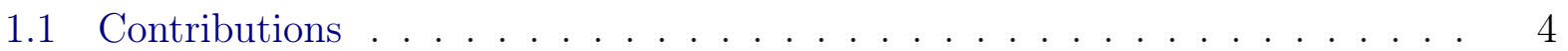

2 Learning Genetic and Environmental Graphical Models from Family Data 5

2.1 Introduction . . . . . . . . . . . . . . . . . . 5 5

2.2 Probabilistic Graphical Models - a Bit of Theory . . . . . . . . . . . . . . 8

2.2.1 Undirected Probabilistic Graphical Models . . . . . . . . . . . . . . . 9

2.2.2 Directed Acyclic Probabilistic Graphical Models . . . . . . . . . . . . 10

2.3 Learning Gaussian PGMs from Independent Observational Units . . . . . . . 12

2.3.1 Partial Correlation for Independent Gaussian Observations . . . . . . 12

2.3.2 Learning Undirected Gaussian PGMs . . . . . . . . . . . . . . . . . . 13

2.3.3 Learning Directed Acyclic Gaussian PGMs . . . . . . . . . . . . . . . 14

2.4 Learning Gaussian PGMs from Family Data . . . . . . . . . . . . . . . . 16

2.4.1 Confounding, Paradoxes, and Fallacies . . . . . . . . . . . . 16

2.4.2 Partial Correlation for Family Data . . . . . . . . . . . . . . . . . 18

2.4.2.1 Confounded Residuals and Predicted Random Effects . . . . 21

2.4.3 Structure Learning from Gaussian Family Data . . . . . . . . . . . . 23

2.4.4 FamilyBasedPGMs R Package . . . . . . . . . . . . . . . 24

2.5 Simulation Study . . . . . . . . . . . . . . . . . . . . 25

2.5.1 Simulation Results . . . . . . . . . . . . . . . 26

2.6 Application to GAW 13 Simulated Data . . . . . . . . . . . . . 27

2.7 Application to Baependi Heart Study Dataset . . . . . . . . . . . . . . . . . 32

2.8 Discussion and Perspectives for Future Work . . . . . . . . . . . . . . . 37

3 Granger Causality Between Graphs $\quad 41$

3.1 Introduction . . . . . . . . . . . . . . . . . . . . . . . . . 41

3.2 Materials and Methods . . . . . . . . . . . . . . . . . . . . 43

3.2.1 Preliminary Concepts . . . . . . . . . . . . . . . 43

3.2.1.1 Graph and Spectral Radius of the Adjacency Matrix . . . . 43

3.2.1.2 Random Graph Models . . . . . . . . . . . . . . . . . . . 44

3.2.1.3 Centrality Measures . . . . . . . . . . . . . . . 46

3.2.1.4 Granger Causality . . . . . . . . . . . . . . 48 
3.2.2 Vector Autoregressive Model for Graphs . . . . . . . . . . . . . . . . 49

3.2.3 Granger Causality Tests . . . . . . . . . . . . . . . . . . 52

3.2.3.1 Wald's Test . . . . . . . . . . . . . . 52

3.2.3.2 Bootstrap Procedure . . . . . . . . . . . . . 53

3.2.4 Implementation in the StatGraph R Package . . . . . . . . . . . . . . 54

3.2.5 Simulation Study . . . . . . . . . . . . . . . . . . . 54

3.2.6 Brain-Brain Interaction Between Two Professional Violinists . . . . . 55

3.2.6.1 Dataset Description and Pre-Processing . . . . . . . . . 55

3.2.6.2 Granger Causality Between Whole-Brain Functional Networks 56

3.2.7 Identifying Differential Connectivity Between Left and Right Brain

Hemispheres in Autistic Subjects . . . . . . . . . . . . . 57

3.2.7.1 ABIDE I Dataset . . . . . . . . . . . . . . 57

3.2.7.2 Data Pre-Processing . . . . . . . . . . . . . 58

3.2.7.3 Granger Causality Between Left and Right Brain Hemispheres 58

3.3 Results and Discussions . . . . . . . . . . . . . . . . . . . . . 60

3.3.1 Simulation Results . . . . . . . . . . . . . . . . . . . 60

3.3.2 Brain-Brain Interaction Between Two Professional Violinists . . . . . 62

3.3.3 Differential Connectivity Between Left and Right Brain Hemispheres in Autistic Subjects . . . . . . . . . . . . . . . . . 65

3.4 Conclusion . . . . . . . . . . . . . . . . . . . . . 67

$\begin{array}{lll}4 & \text { Conclusions } & 71\end{array}$

$\begin{array}{ll}\text { Bibliography } & 75\end{array}$ 


\section{Chapter 1}

\section{Introduction}

Causality plays a central role in science, providing scientific explanations of behaviors and phenomena. Particularly, in biological systems, the knowledge of the causal network describing the relations among multiple traits and phenotypes may help us to better understand the underlying mechanisms and the risk factors of diseases.

A definition of causality that is intimately linked with the manipulability (capacity to intervene) was established mainly by Von Wright (1971), who gave the influential statement that $A$ is a cause of $B$ if and only if by manipulating $A$ we can achieve or bring it about $B$ is, or is not, there.

That notion of causality is similar to that for randomized controlled trials (RCTs), in which any change in the outcome variable is expected to be due to the intervention, if all factors influencing their association are either constant, or vary at random. Also, is the foundation of structural equation modeling and probabilistic graphical modeling, both exploited by Pearl (2000) and Spirtes et al. (2000) to provide a formulation of the manipulability theory of causation in terms of interventions. In recent literature, this experimentalist or interventionist theory of causation has also been developed by Woodward (2005).

Although RCTs are widely accepted as the most trustworthy method for disentangling causal effects, it is generally impracticable to perform all necessary experiments because they are expensive and time consuming. Causal learning and inference from purely observational data is a notoriously difficult problem, but under certain assumptions, it is possible to drawn reliable causal conclusions.

In Pearl's theory of causation (Pearl, 2000), probabilistic graphical models (PGMs) are used to provide a complete characterization of causal effects in terms of conditional independences among the variables. Based on a conditional independence test, constraintbased structure learning algorithms, such as the IC/PC algorithm (Pearl and Verma, 1991; Spirtes et al., 2000), can learn about the structure of the PGM from observational data if the assumptions are satisfied. For multivariate Gaussian variables and independent observations, conditional independence is equivalent to zero partial correlation (Lauritzen, 1996, Proposition 5.2), so Gaussian PGMs can be learned by performing multiple zero partial 
correlation tests.

In Genetics, besides understanding how the variables are related to each other, it is crucial to unravel how the connections among the variables are influenced by genetic and environmental factors. Family-based studies have proven to be useful in elucidating genetic and environmental factors affecting variables, since familial clustering may reveal genetic effects (Almasy and Blangero, 1998; Amos, 1994; De Andrade et al., 2015; Lange, 2003; Oualkacha et al., 2012). However, few existing approaches are able to address structure learning of PGMs and family data analysis jointly.

In Chapter 2, we propose methods for learning, from observational Gaussian family data, the structure of the most likely PGM over multiple phenotypes and also its decomposition into two components: one explained by a polygenic (between-family) factor and other explained by other factors, which we are calling environmental (within-family) factor.

In family data, measurements from related individuals are correlated, so the classical zero partial correlation test, which is valid only for independent observations, is inappropriate for assessing conditional independence among the variables. Thus, in Section 2.4.2, based on univariate polygenic linear mixed models (Almasy and Blangero, 1998; Amos, 1994; Lange, 2003), we derive unconfounded genetic and environmental partial correlation estimators and proper zero partial correlation tests for these coefficients, taking into account the familial dependence structure. Then, we used these tests to extend classical structure learning algorithms to deal with Gaussian family data.

The proposed methods were evaluated by a simulation study as described in Section 2.5. In Section 2.6, it is shown an application to the Genetic Analysis Workshop 13 simulated data (Daw et al., 2003), which capture the major features of the Framingham Heart Study (Levy et al., 2000). Also, with the aim of understanding the mechanism behind the metabolic syndrome phenotypes and how it is affected by genetic and environmental factors, in Section 2.7 we applied the proposed methods to phenotypic data from the Baependi Heart Study (de Oliveira et al., 2008), a very large Brazilian family-based cohort study designed to investigate cardiovascular disease and associated factors.

In neuroscience, the brain functional connectivity is usually studied by constructing functional brain networks (graphs) in which the vertices represent brain regions of interest and the edges represent functional connectivity between those regions. However, these functional networks are dynamic and current methods typically ignore a key component: time.

Another probabilistic definition of causation was given by Granger (1969). Based on the concept of predictability and on the temporal precedence of the cause over the effect, Clive Granger adapted the definition of causality by the mathematician Norbert Wiener (Wiener, 1956) into a more practical form, known as Wiener-Granger causality or simply Granger causality. We say that a stochastic process $y_{1, t}$ Granger causes another stochastic process $y_{2, t}$ if past information on $y_{1, t}$ helps in forecasting $y_{2, t}$ even accounting for the information on the past of $y_{2, t}$ and also of other possible explanatory variables. This notion of causality allows a feedback stochastic system. For example, it is possible that $y_{1, t}$ Granger causes $y_{2, t}$ 
as well as $y_{2, t}$ Granger causes $y_{1, t}$.

Its mathematical formulation is often based on the vector autorregressive (VAR) modeling, in which each stochastic process is linearly regressed on $p$ of its own past values and also on $p$ past values of all the other variables.

In many biological systems, the observed data have naturally a dependence structure that can be represented by a graph. For example, the brain regions have been characterized according to their anatomical and functional properties by several studies. Thus, by including this information in the analysis, results can be more easily interpreted. In addition, it is known that these brain regions are not static. The connections within each region are constantly changing. To understand the interdependence structure between the brain regions taking into account their intrinsic structural and dynamical properties, we propose a method to identify Granger causality between graphs.

Since it is hard to incorporate the graph structure in the VAR model, we used ideas similar to those of Fujita et al. (2017) to provide a new notion of Granger causality between graphs. Considering two stochastic processes whose realizations are, respectively, the time series of graphs $y_{1, t}$ and $y_{2, t}$, and assuming that the graphs are generated by models whose parameters are random variables (under a Bayesian formulation), we define that $y_{1, t}$ does not Granger cause $y_{2, t}$ if the model parameters for $y_{1, t}$ do not Granger cause the model parameters $y_{2, t}$.

The problem is that the models that generate the graphs, and consequently the model parameters, are unknown. For some random graph models, such as Erdös-Rényi, geometric, regular, Watts-Strogatz, and Barabási-Albert, it is known that the spectral radius (the largest eigenvalue of the adjacency matrix of the graph) is a function of the model parameters (Alon, 1986; Bordenave, 2008; Dorogovtsev et al., 2003; Füredi and Komlós, 1981; Van Mieghem, 2010). For example, for the Erdös-Rényi random graph model, which is defined by the parameters $n$, number of vertices, and $p$, probability of two random vertices are connected, the spectral radius converges to $n p-2 p+1$ (Füredi and Komlós, 1981). Thus, information on the parameters of the model that generates the graph can be captured by the spectral radius.

Based on this idea, we propose in Chapter 3 a method called gVAR, that identifies Granger causality between time series of graphs by fitting a VAR model to the time series of the ranked spectral radii. The rank transformation of the spectral radii is suggested to allow detection of monotonic relationships, to increase the robustness to outliers, and to eliminate problems with asymmetric distributions. A detailed explanation of the gVAR method is provided in Section 3.2.2.

By the simulation study described in Section 3.2.5, we show in Section 3.3.1 that the method has good accuracy, particularly for large graphs and long time series. In addition, by using the spectral radius as a feature of the graph, the method produces better results than using other centrality measures, such as degree, eigenvector, betweenness, and closeness centralities. We illustrate the application of the method by studying brain-brain interaction 
of two professional musicians when playing a violin duo while being measured from the same Functional Near Infrared Spectroscopy (fNIRS) system. In Section 3.3.2, we show that the whole-brain functional network of the older and more experienced violinist Granger causes the whole-brain functional network of the other violinist. In addition, with the aim of studying Granger causality between functional networks of the left and right brain hemispheres in subjects with autism spectrum disorder (ASD) and typical controls, we applied the proposed method to the Autism Brain Imaging Data Exchange I (ABIDE I) dataset. We show in Section 3.3.3 that the Granger causality from the right to the left hemisphere decreases with age in subjects with ASD, but not in typical controls.

\subsection{Contributions}

The main contributions of this work are as follow:

- In Chapter 2, we developed algorithms for learning from observational Gaussian family data the most likely PGM over a set of variables and its decomposition into polygenic (between-family) and environmental (within-family) components.

The algorithms are implemented in $\mathrm{R}$ and available at the FamilyBasedPGMs $\mathrm{R}$ package on GitHub: https://github.com/adele/FamilyBasedPGMs.

- In Chapter 3, we developed an algorithm that identifies Granger causality between graphs.

The gVAR method is implemented in $\mathrm{R}$ and will be available soon as part of the statGraph R package at CRAN: https://cran.r-project.org/package=statGraph.

- A book chapter describing the main concepts of the Pearl's theory of causation and algorithms for learning genotype-phenotype networks using genetic variants was published by Springer and available at http://www.springer.com/br/book/9783319412788 (Ribeiro et al., 2016). 


\section{Chapter 2}

\section{Learning Genetic and Environmental Graphical Models from Family Data}

To unravel the mechanism underlying traits and diseases, it is crucial to understand how variables are related to each other and influenced by genetic and environmental factors. Probabilistic graphical models (PGMs) are widely used to describe relationships among multiple variables in a very intuitive and mathematically rigorous way. Family-based studies have proven to be useful in elucidating genetic and environmental factors affecting variables, since familial clustering may reveal genetic effects. In this work, we propose to learn the structure of PGMs from observational Gaussian family data. Many structure learning algorithms are strongly based on a conditional independence test. For independent data and normally distributed variables, conditional independence can be tested using classical zero partial correlation tests. However, in family data, measurements from related individuals are correlated and such dependence structure has to be accounted for in statistical tests. Based on univariate polygenic linear mixed models, we derive proper zero partial correlation tests that allow to extend classical structure learning algorithms, which rely on the assumption of independent observations, to learn from Gaussian family data. In addition, we provide a decomposition of the learned PGM into polygenic (between-family) and environmental (within-family) components. The proposed methods were evaluated by a simulation study and applied to the Genetic Analysis Workshop 13 simulated data, which capture the major features of the Framingham Heart Study, and also to the metabolic syndrome phenotypes from the Baependi Heart Study, a Brazilian family-based cohort.

\subsection{Introduction}

A major goal of biomedical research is to better understand disease mechanisms and biological systems for improving diagnosis, prognostic information, and therapies. To intervene effectively in the system, it is essential to recover the network of relationships (causal or not) among the related multiple traits and phenotypes, and to understand how these 
relationships are influenced by genetic and environmental factors.

Association and, particularly, causation studies from observational data are challenging because an observed statistical association between two variables may arise not only from the effect of one variable on another but also from confounding factors and selection bias. Experimental studies, such as randomized controlled trials (RCTs), are widely accepted as the most trustworthy method for disentangling causal effects. However, because they are expensive and time consuming, it is generally impracticable to perform all necessary experiments. Thus, faced with the large amounts of data and information available from observational studies, such as electronic health records, several efforts have been made to bridge the gap between statistical dependency and causality, showing that it is possible to recover the structure of causal networks and to infer causal effects based on observational data alone if certain assumptions are met.

Pearl's theory of causation (Pearl, 2000), which unifies the potential-outcome framework (Rubin, 1974), probabilistic graphical models (PGMs) (Koller and Friedman, 2009), and structural equation models (SEMs) (Bollen, 1989) is a remarkable example of formalization of causal reasoning. Also, Pearls's interpretation of causation is similar to that for RCTs, in which any change in the outcome variable must be due to the intervention, if all factors influencing their association are either constant, or vary at random. Particularly, PGMs are a powerful framework that combines graph and probability theories to graphically represent the (conditional) independence relations among variables and, thus, to provide a simplified factorization of the joint probability distribution over the variables. They were proposed as early as Dempster (1972) for covariance selection of multivariate Gaussian distributions, but started to be more popular after Pearl (1988) and Lauritzen and Spiegelhalter (1988), and particularly after Whittaker (1990) and Lauritzen (1996).

Causal PGMs are represented by directed acyclic graphs (DAGs). The directionality of the edges is used to convey causal (functional) relationships. Considering only linear relationships, a causal PGM precisely corresponds to a linear SEM and its graphical representation is the path diagram introduced by Wright (1921). Other graphical tools developed within the PGM framework, such as graph separation, are intended for non-parametric analysis, but for linear relationships, they provide simple and meaningful interpretation of the causal effects as well as the basis for model testing and identification criteria (Chen and Pearl, 2014).

Since PGMs represent conditional independence relation between the variables, the structure of both undirected and directed PGMs can be learned by algorithms, known as constraintbased structure learning algorithms, that perform many conditional independence tests.

In the context of quantitative genetics, family data are analyzed by using polygenic models (Almasy and Blangero, 1998; Amos, 1994; Lange, 2003), which are linear mixed models with two components of variance: the polygenic or family-specific random effect, which models the phenotypic variability across the families, and the environmental or subject-specific error, which models phenotypic variability after removing the familial aggregation effect. Thus, (co)variance of phenotypes may be partitioned according to genetic and environmen- 
tal influences, elucidating the nature of their mechanisms. For a multivariate analysis of multiple traits, the multivariate polygenic mixed model can be used (De Andrade et al., 2015; Oualkacha et al., 2012).

Although structure learning of PGMs and family data analysis have been widely addressed in isolation, few existing approaches are capable of addressing both aspects jointly.

Existing approaches for learning undirected PGMs from family data, such as Dahl et al. (2013) and Talluri and Shete (2014), are based on the graphical lasso (Friedman et al., 2008) algorithm, which learns the sparse undirected Gaussian PGM by estimating the sparse inverse covariance matrix (also known as precision or concentration matrix). Talluri and Shete (2014) present an approach for learning the marginal or total-sample graph for the Genetic Analysis Workshop (GAW) 18 data. The marginal inverse covariance matrix is estimated taking into accounting the correlation structure among individuals using the kinship coefficients. Dahl et al. (2013) use a multivariate linear mixed model to estimate the marginal covariance matrix as a sum of a genetic covariance matrix, structured according to the relatedness information among the individuals, and a residual covariance matrix. Also, they incorporate an $\ell_{1}$ penalty on the entries of both genetic and residual covariance matrices to impose sparsity.

Considering the correspondence between causal PGMs and SEMs for linear causal relationships, Valente et al. (2010) propose an approach for learning causal PGMs from pedigree data, by modeling the phenotypes by a recurrent SEM with additive genetic (polygenic) and residual covariance structures. Their approach consists in first rewrite the SEM as a multivariate mixed model, under its reduced model formulation, and then estimate the additive genetic and residual covariance matrices by using Bayesian techniques. The relationships among phenotypes are learned by applying classical causal structure learning algorithms on the estimates of these covariance matrices. Initially, Valente et al. (2010) considered the additive genetic covariances as confounders and learned the causal structure only considering the residual covariance matrix. Later, Töpner et al. (2017) applied the method for learning both additive genetic and residual PGMs, arguing that they may be different, since the connections in the latter are mainly due to environmental causes.

Many structure learning algorithms are strongly based on a conditional independence test. When the observations are independent and normally distributed, the null hypothesis of conditional independence can be tested using classical tests for zero partial correlation (Lauritzen, 1996, Proposition 5.2). The partial correlation coefficient between two variables can be estimated by first linearly regressing each of these two variables on the conditioning variables, and then computing the Pearson correlation between the residuals of these two classical univariate regressions. However, in family data, observations from relatives may be correlated, so the independence assumption is no longer satisfied.

In this work, we develop appropriate partial correlation estimators that take into account the dependence structure among individuals, based on univariate polygenic mixed models. Considering the maximum likelihood (ML) or restricted maximum likelihood (REML) es- 
timators, the conditional residuals and the predicted random effects are known to be confounded (Hilden-Minton, 1995). Thus, inspired by Loy and Hofmann (2015), we propose a strategy to minimize this confounding, allowing to perform zero partial correlation tests separately on the polygenic and environmental components. Based on these tests and under the additional assumptions of linearity and homoscedastic families, we develop methods for learning, from observational Gaussian family data, the undirected and directed PGM that describe, respectively, associations or causal relationships among multiple phenotypes and, more importantly, a decomposition of the learned PGM into unconfounded polygenic and environmental PGMs. Since our approaches learn multivariate distributions by fitting only univariate mixed models, we have a lot of flexibility and scalability to easily develop and implement further improvements.

By a simulation study, we showed that the proposed methods can recover the true network with good accuracy, even in situations where the polygenic and environmental components are highly confounded. Also, we show an application to the simulated data from the GAW 13 (Daw et al., 2003), which were generated so that major features of the real Framingham Heart Study data (Levy et al., 2000) are captured. Finally, to learn the genetic and environmental PGMs for the metabolic syndrome phenotypes, we analyzed the data from the Baependi Heart Study, a family-based cohort study conducted in a rural Brazilian town (de Oliveira et al., 2008).

In Section 2.2, we briefly introduce the theory of the PGMs. In Section 2.3 we review some existing methods for learning Gaussian PGMs considering independent data and in Section 2.4 we show how the methods can be extended for learning PGMs from family data. Specifically, in Section 2.4.2, we describe unconfounded estimation and significance test for the genetic and environmental partial correlation. Simulation studies are considered in Section 2.5 and an application using GAW 13 data is shown in Section 2.6. The application to the Baependi Heart Study data is shown in Section 2.7. Finally, discussion and perspectives for future work are in Section 2.8.

\subsection{Probabilistic Graphical Models - a Bit of Theory}

PGMs (also known as Conditional Independence Graphs) are a mathematically rigorous and intuitive language for encoding the (conditional) independence relations existing in the joint probability distribution over a set of variables.

The joint probability distribution $P$ over a set of variables $\boldsymbol{V}$ can be represented by an undirected or a directed graph (usually acyclic), in which a vertex represents a variable in $\boldsymbol{V}$ and a missing edge between two variables, $X, Y \in \boldsymbol{V}$, implies that there exists a subset of other variables, $\boldsymbol{S} \subseteq \boldsymbol{V} \backslash\{X, Y\}$, such that $X$ and $Y$ are independent when conditioned on $\boldsymbol{S}$ (we write $X \Perp Y \mid \boldsymbol{S}$ ). Undirected PGMs are also know as Markov Random Fields or Markov Networks, and Directed Acyclic PGMs are also know as Bayesian or Causal Networks 
(Koller and Friedman, 2009).

We say that $P$ is perfectly encoded by a PGM $G$ if every (conditional) independence relation in $P$ is represented by a missing edge in $G$ and if every missing edge in $G$ encodes a (conditional) independence relation holding in $P$. In this case, we also say that $G$ is a perfect map of $P$ (Pearl, 2000).

In Sections 2.2.1 and 2.2.2 we define, respectively, for undirected and directed acyclic PGM, the (d-)separation criterion and two necessary and sufficient conditions to establish this equivalence between $G$ and $P$ : the faithfulness condition and the global Markov property. The global Markov property implies that all (d-)separation statements obtained from $G$ hold as conditional independence statements in $P$, while the faithfulness guarantees the converse, that is, that all conditional independence statements implied by $P$ can be read off from $G$.

Spirtes et al. (2000) showed that almost all multivariate Gaussian distributions that satisfy the global Markov property with respect to a certain undirected or directed acyclic graph are faithful, i.e., the set of parameterizations in which the distribution is unfaithful has Lebesgue measure zero. In addition, the Hammersley-Clifford theorem shows that the global Markov property is equivalent to other weaker properties if the probability distribution has a strictly positive density function, as is the case for multivariate Gaussian distributions. These properties are described in the next sections. An exposition on that theory can be found at Ribeiro et al. (2016).

\subsubsection{Undirected Probabilistic Graphical Models}

We say that $P$ satisfies the global Markov property for an undirected PGM $G$ if, for all disjoints sets of variables $\mathbf{X}, \mathbf{Y}$, and $\mathbf{Z}$, it holds that, if $\mathbf{X}$ is separated from $\mathbf{Y}$ given $\mathbf{Z}$ in $G$ (i.e., every path between a vertex in $\mathbf{X}$ and a vertex in $\mathbf{Y}$ passes through a vertex in $\mathbf{Z}$ ), then $\mathbf{X}$ is conditionally independent from $\mathbf{Y}$ given $\mathbf{Z}$ in $P$. Thus, the following properties also hold:

- Pairwise Markov property: a missing edge in $G$ between two vertices implies that in $P$ these two vertices are conditionally independent given all the other variables;

- Local undirected Markov property: every vertex is independent of the other vertices given its direct neighbors;

- Clique factorization: $P$ factorizes in terms of non-negative functions, called clique potentials, which depend only on the variables in their maximal cliques (fully-connected subgraphs). Let $\mathcal{C}$ be the set of all maximal cliques in $G$ and $\left\{\psi_{C}\left(V_{C}\right) \geq 0\right\}_{C \in \mathcal{C}}$ be the clique potentials. By enumerating the variables in $\boldsymbol{V}$ as $\left\{V_{1}, \ldots, V_{p}\right\}, P$ can be written as the following product of the clique potentials:

$$
P\left(V_{1}, \ldots, V_{p}\right)=\frac{1}{Z} \prod_{C \in \mathcal{C}} \psi_{C}\left(V_{C}\right),
$$


where $Z$ is a normalizing constant defined as:

$$
Z=\int_{V_{1}, \ldots, V_{p}} \prod_{C \in \mathcal{C}} \psi_{C}\left(V_{C}\right) \mathrm{d} V_{1}, \ldots, \mathrm{d} V_{p}
$$

Note that the pairwise Markov property is weaker than the local undirected Markov property, which is weaker than the global Markov property.

Let, for example, $P$ be the joint distribution over $\boldsymbol{V}=\{X, Y, Z, W\}$, where $X \Perp$ $Z \mid\{W, Y\}$ and $Y \Perp W \mid\{X, Z\}$. The undirected PGM shown in Figure 2.1-A is the only PGM that perfectly encodes $P$ and, thus, $P$ factorizes as:

$$
P(X, Y, Z, W) \propto \psi(X, Y) \psi(X, W) \psi(Y, Z) \psi(W, Z)
$$

\subsubsection{Directed Acyclic Probabilistic Graphical Models}

In this work, we consider only directed acyclic PGMs, which correspond to recursive SEMs (those with uncorrelated errors and without cyclic or feedback effects), and are referred by DAGs.

We say that $P$ satisfies the global Markov property for a directed acyclic PGM $G$ if, for all disjoints sets of variables $\mathbf{X}, \mathbf{Y}$, and $\mathbf{Z}$, it holds that, if $\mathbf{X}$ is d-separated from $\mathbf{Y}$ given $\mathbf{Z}$ in $G$ (i.e., all undirected paths between a vertex in $\mathbf{X}$ and a vertex in $\mathbf{Y}$ are blocked, either because the path has a collider - a vertex with edges pointing to it from two directions - such that neither it, nor any of its descendants, is in $\mathbf{Z}$, or because the path contains a non-collider which is in $\mathbf{Z}$ ), then $\mathbf{X}$ is conditionally independent from $\mathbf{Y}$ given $\mathbf{Z}$ in $P$. Thus, the following properties also hold:

- Local directed Markov property: every vertex is independent of all other nondescendants (all other vertices except its parents and descendants) given its parents.

- Recursive factorization: by enumerating the variables in $\boldsymbol{V}$ as $\left\{V_{1}, \ldots, V_{p}\right\}, P$ factorizes as the product of the conditional probabilities of each variable $V_{i}$, for $i=$ $1, \ldots, p$, given its parents, defined by $p a\left(V_{i}\right)$ :

$$
P\left(V_{1}, \ldots, V_{p}\right)=\prod_{i=1}^{p} P\left(V_{i} \mid p a\left(V_{i}\right)\right) .
$$

Note that the pairwise property does not hold for directed PGMs. A missing edge in a directed PGM $G$ implies that the two disconnected vertices are conditionally independent given some set of other variables. More specifically, when encoding the joint distribution by a directed acyclic PGM, it is not necessarily true that if $X \Perp Y \mid \boldsymbol{S}^{\prime}$, where $\boldsymbol{S}^{\prime} \subset \boldsymbol{S}=$ $\boldsymbol{V} \backslash\{X, Y\}$, then $X \Perp Y \mid \boldsymbol{S}$. In fact, according to the d-separation criterion, $X \not \mathbb{} Y \mid \boldsymbol{S}$ if there is a collider in a path between $X$ and $Y$ and it or any of its descendants is in $\mathbf{S}$. Thus, 
the edge between $X$ and $Y$ is missing only if there exists, among all possible conditioning sets of other variables, at least one set $\boldsymbol{S}^{\prime}$ such that $X \Perp Y \mid \boldsymbol{S}^{\prime}$.

The moral graph of a DAG $G$ is obtained by connecting all parents of a common child in $G$ and by dropping the edge directions. All conditional independence statements implied by the moral graph of $G$ are also implied by the $G$. Thus, if $P$ factorizes according to a DAG $G$, then it also factorizes according to its moral graph. Note that the converse is not always true, i.e., the moral graph may not include all the conditional independence statements implied by $G$.
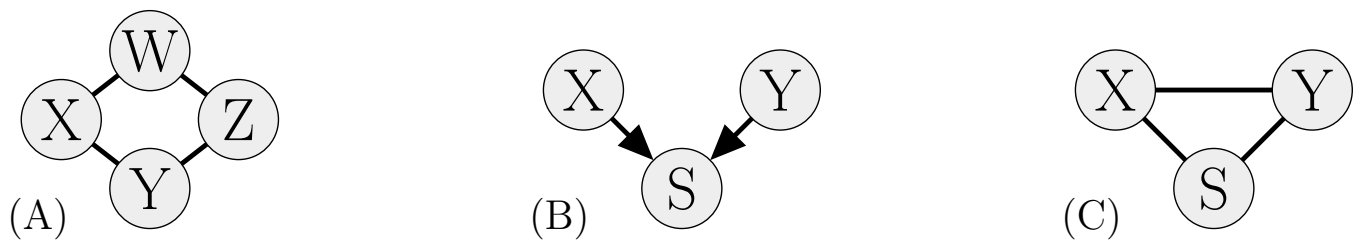

Figure 2.1: (A) Undirected $P G M$ encoding the joint probability distribution over $X, Y, Z$, and $W$, where $X \Perp Z \mid\{W, Y\}$ and $Y \Perp W \mid\{X, Z\}$. (B) Directed acyclic PGM, known as unshielded collider or $v$-structure, encoding the joint probability distribution over $X, Y$, and $S$, where $X \Perp Y$ and $X \not K Y \mid S$. The vertex $S$ is known as collider or common effect of $X$ and $Y$. (C) Moral graph of $(B)$, obtained by connecting the vertices $X$ and $Y$, which are "unmarried" parents of $S$, and by dropping directions.

Let, for example, $P$ be the joint distribution over $\boldsymbol{V}=\{X, Y, S\}$, where $X \Perp Y$ and $X \not 1 Y \mid S$. The only PGM that encodes $P$ is the DAG shown in Fig. 2.1-B, known as $v$-structure or unshielded collider. It is the only connected triplet (a formation of three vertices and two edges) where two variables are marginally independent, but they become dependent when conditioned on the third variable (called collider or common cause, in this case). Therefore, $P$ factorizes according to the DAG in Fig. 2.1-B, i.e.,

$$
P(X, Y, S)=P(X) P(Y) P(S \mid X, Y),
$$

and also factorizes according to its moral graph, shown in Figure 2.1-C. However, as its moral graph is the complete graph (there is only one maximal clique), the implied factorization is not informative. The problem is that the conditional dependence of $X$ and $Y$ given $S$ is reflected by the edge between $X$ and $Y$, but the marginal independence between $X$ and $Y$ is not encoded.

Now, let $P$ be the joint distribution over $\boldsymbol{V}=\{X, Y, S\}$, where $X \not \Perp Y ; \quad X \Perp Y \mid S$. These relations are perfectly encoded by all of the following directed acyclic PGMs shown in Figure 2.2.

By the Bayes's theorem, the directed acyclic PGMs in Figure 2.2 imply factorizations of the same joint distribution, which are, respectively: (A) $P(X, Y, S)=P(X \mid S) P(Y \mid S) P(S)$, (B) $P(X, Y, S)=P(X) P(Y \mid S) P(S \mid X)$, and (C) $P(X, Y, S)=P(X \mid S) P(Y) P(S \mid Y)$.

DAGs that entail the same conditional independence relations are statistically equivalent (represent the same joint distribution) and belong to the same Markov equivalence class. A 

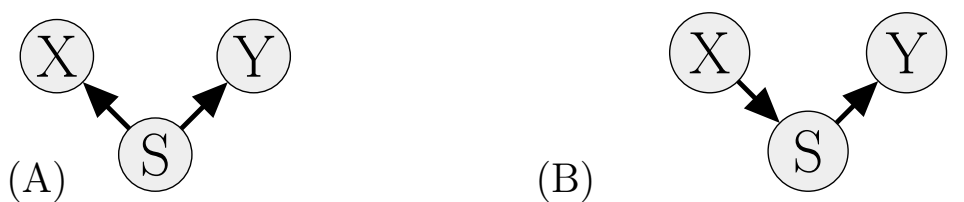

$(\mathrm{C})$

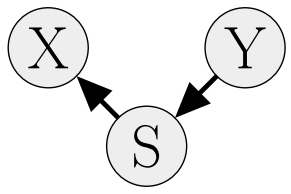

Figure 2.2: The joint probability distribution over the variables $X, Y$, and $S$, where $X \not K Y$ and $X \Perp Y \mid S$, can be perfectly encoded by three different directed acyclic PGMs: (A) a fork, where the vertex $S$ is known as the common cause of $X$ and $Y$; $(B)$ a direct chain; and $(C)$ a reverse chain.

theorem by Pearl and Verma (1990) states that, under the assumption of acyclicity, DAGs are Markov equivalent if and only if they have the same skeleton (the undirected version of the graph) and the same set of unshielded colliders.

\subsection{Learning Gaussian PGMs from Independent Obser- vational Units}

When a PGM precisely encodes a multivariate Gaussian distribution under independent observations, it is called Gaussian PGM. For this distribution, the Hammersley-Clifford theorem (Hammersley and Clifford, 1971; Lauritzen, 1996) states that all Markov and factorization properties are equivalent, thus, structure learning algorithms can search for the true graph by only verifying the weaker Markov properties. For undirected Gaussian PGMs, structure learning algorithms can verify only the local undirected Markov property or even only the pairwise Markov property. And for directed acyclic Gaussian PGMs, algorithms can verify only the local directed Markov property.

Since those searches are strongly based on conditional independence constraints, these structure learning algorithms are called conditional independence learners or constraint-based algorithms. In these cases, a conditional independence test is used as a separation oracle that answers whether a conditional independence relation holds or not. For multivariate Gaussian variables, conditional independence is equivalent to zero partial correlation (Lauritzen, 1996, Proposition 5.2), so Gaussian PGMs can be learned by performing many zero partial correlation tests.

Note that, since multiple hypothesis tests (although they are very unlikely to be independent of each other) are conducted for learning the structure of the PGM, the chance of committing a Type I error increases. Therefore, the reliability of the learned PGM critically depends on the choice of the significance level. So, a correction for large-scale multiple hypothesis testing should be considered.

\subsubsection{Partial Correlation for Independent Gaussian Observations}

Let $\boldsymbol{V}$ be a random vector following a multivariate normal distribution. Consider two variables $X, Y \in \boldsymbol{V}$ and a set $\boldsymbol{S} \subseteq \boldsymbol{V} \backslash\{X, Y\}$. The partial correlation coefficient between $X$ 
and $Y$ given $\mathbf{S}$, denoted by $\rho_{X Y \mid \mathbf{S}}$, is a measure of the degree of association between $X$ and $Y$ after removing any association that these two variables have with the variables in $\boldsymbol{S}$. So, under the assumption that the variables are linearly associated and considering a sample of $N$ independent observations $\left(\boldsymbol{V}_{i}, i=1, \ldots, N\right)$, the partial correlation $\rho_{X Y \mid \mathbf{S}}$ can be estimated by the Pearson correlation between the residuals of the following univariate linear regression models (Anderson et al., 1958; Meinshausen et al., 2006):

$$
\begin{array}{cl}
x_{i}=\alpha^{X}+\sum_{k=1}^{|\mathbf{S}|} \beta_{k}^{X} s_{k i}+\varepsilon_{i}^{X}, & \varepsilon_{i}^{X} \sim \mathcal{N}\left(0, \sigma_{X \mid S}^{2}\right), \\
y_{i}=\alpha^{Y}+\sum_{k=1}^{|\mathbf{S}|} \beta_{k}^{Y} s_{k i}+\varepsilon_{i}^{Y}, & \varepsilon_{i}^{Y} \sim \mathcal{N}\left(0, \sigma_{Y \mid \boldsymbol{S}}^{2}\right),
\end{array}
$$

for $i=1, \ldots, N$, where $x_{i}$ is the $i$ th observation of the variable $X, \alpha^{X}$ is the overall mean, $\beta_{k}^{X}$ and $s_{k i}$, for $k=1, \ldots,|\boldsymbol{S}|$, are, respectively, the parameters and the $i$ th observations for the variables in $\boldsymbol{S}$, and $\varepsilon_{i}^{X}$ is the $i$ th error term in the regression for $X$. The notation is analogous in the regression for $Y$. Thus, denoting by $\hat{\varepsilon}^{X}$ and $\hat{\varepsilon}^{Y}$, the ordinary least square (OLS) estimates of $\varepsilon^{X}$ and $\varepsilon^{Y}$, respectively, the sample partial correlation coefficient can be estimated as follows:

$$
\hat{\rho}_{X Y \mid \mathbf{S}}=\operatorname{corr}\left(\hat{\varepsilon}^{X}, \hat{\varepsilon}^{Y}\right) .
$$

The Fisher's $\mathrm{Z}$ test can be used to test the null hypothesis $H_{0}: \rho_{X Y \mid \mathbf{S}}=0$ against the two-tail alternative hypothesis $H_{1}: \rho_{X Y \mid \mathbf{S}} \neq 0$. Consider the Fisher's z-transformed sample partial correlation coefficient:

$$
z\left(\hat{\rho}_{X Y \mid \mathbf{S}}\right)=\frac{1}{2} \ln \left(\frac{1+\hat{\rho}_{X Y \mid \mathbf{S}}}{1-\hat{\rho}_{X Y \mid \mathbf{S}}}\right)
$$

Then, the statistic

$$
\sqrt{N-|\mathbf{S}|-3}\left|z\left(\hat{\rho}_{X Y \mid \mathbf{S}}\right)\right|
$$

asymptotically follows, under $H_{0}$, a standard normal distribution.

\subsubsection{Learning Undirected Gaussian PGMs}

Let $\boldsymbol{V}$ be a $p$-dimensional Gaussian vector with mean $\boldsymbol{\mu}_{p}$ and covariance matrix $\boldsymbol{\Sigma}_{p \times p}$. Considering that, for multivariate normal distributions, the pairwise Markov property implies the global Markov property, the standard algorithm for learning undirected Gaussian PGMs assigns an edge between every pair of variables $(X, Y)$ such that the null hypothesis $H_{0}: \rho_{X Y \mid \boldsymbol{V} \backslash\{X, Y\}}=0$ is rejected (Lauritzen, 1996; Whittaker, 1990). Thus, for learning undirected PGM among the $p$ variables in $\boldsymbol{V}$, it is necessary to compute $p(p-1) / 2$ partial correlation coefficients.

One common approach is to estimate all coefficients directly from the $p \times p$ sample inverse 
covariance matrix, denoted by $\boldsymbol{K}=\hat{\boldsymbol{\Sigma}}_{p \times p}^{-1}$. Denoting by $k_{X Y}$ the entry of $\boldsymbol{K}$ associated to the concentration between the variables $X, Y \in \boldsymbol{V}$, we have:

$$
\hat{\rho}_{X Y \mid S}=\frac{-k_{X Y}}{\sqrt{k_{X X} k_{Y Y}}}
$$

Note that $X$ and $Y$ are conditionally independent given all other variables in $\boldsymbol{V}$ if and only if the entry $k_{X Y}$ is zero:

$$
X \Perp Y \mid \boldsymbol{V} \backslash\{X, Y\} \Leftrightarrow k_{X Y}=0 .
$$

Thus, the undirected Gaussian PGM is recovered by assigning edges between every pair of variables such that the corresponding entry of the sample inverse covariance matrix is non-zero. For that reason, the structure learning problem for undirected Gaussian PGMs is also known as covariance selection (Dempster, 1972).

Another approach is to use the partial correlation estimator based on univariate linear regressions, shown in Section 2.3.1. Consider the $N \times p$ data matrix with $N$ independent observations of $\boldsymbol{V}$ and its vectorization, $\boldsymbol{v}$, obtained by stacking its rows. Denote by $\mathbb{1}_{N}$ the unit vector, by $\mathbf{I}_{N}$ the identity matrix of order $N$, and by $\otimes$ the Kronecker product. The undirected PGM that perfectly encodes the multivariate Gaussian distribution

$$
\boldsymbol{v} \sim \mathcal{N}_{N p}\left(\mathbb{1}_{N} \otimes \boldsymbol{\mu}_{p}, \mathbf{I}_{N} \otimes \boldsymbol{\Sigma}_{p \times p}\right)
$$

can be learned by just fitting univariate regression models.

In recent years, there has been a growing interest in structure learning approaches based on univariate regression models because of all the flexibility and scalability provided by them. For example, by imposing $l_{1}$ penalty (Lasso) on the regressions, it is possible to learn even from high-dimensional data. The most famous is the neighborhood selection approach by Meinshausen et al. (2006), that learns the structure of the undirected graphical model by estimating the conditional independence restrictions separately for each vertex in the graph. These algorithms have been shown to have strong computational as well as statistical guarantees.

\subsubsection{Learning Directed Acyclic Gaussian PGMs}

Algorithms for learning acyclic directed PGMs, also known as causal structure learning algorithms, are mainly grouped in two categories: score-based and constraint-based approaches (Koller and Friedman, 2009).

Score-based algorithms recover the most likely structure (the one or a member of a group of structures with highest score of goodness-of-fit) by performing a heuristic search in the space of all possible structures (Chickering, 2002; Tsamardinos et al., 2006). In this work, we are interested in constraint-based algorithms, which obtain information about the 
underlying causal structure by performing many conditional independence tests and are all derived from the IC (Inductive Causation) algorithm (Pearl and Verma, 1991) and from its refined version PC-algorithm (after its authors, Peter and Clark) (Spirtes et al., 2000).

Structure learning algorithms are not able to distinguish between graphs that entail exactly the same set of conditional independence relations, i.e. between Markov equivalent graphs. In particular, the IC/PC algorithm learns, under the assumptions of acyclicity, faithfulness, causal sufficiency (no unobserved common causes of the measured variables), and reliable conditional independence tests, a completed partially directed acyclic graph (CPDAG), which, under an adopted significance level, uniquely represents the Markov equivalence class in which the true DAG belongs. In this representation, a directed edge indicates that all DAGs belonging to the equivalence class have this edge oriented in that same direction, while an undirected edge indicates that some DAGs have this edge directed on one way, while other DAGs have it directed on the other way.

The IC/PC algorithm starts with a complete undirected graph. Then, for each pair of variables, it searches, among all possible conditioning sets, for one that makes the two variables conditionally independent. If it exists, then it means that the dependence between the two variables can be explained away and the edge connecting the pair can be removed. Technically, we say that the two variables are d-separated in the graph by such conditioning set. After that adjacency phase, the skeleton of the graph has been inferred and the algorithm goes to the orientation phase. Since the unshielded colliders are the only connected triplets that can be uniquely oriented, they are the first to be oriented. After orienting all unshielded colliders, the algorithm tries to orient the other edges by consistency, without creating any loop or new colliders. That is recursively made until no more edges can be oriented.

If the true underlying DAG is sparse (the best case scenario), the time complexity of the IC/PC algorithm is polynomial. However, if the true underlying DAG is not sparse (worst case scenario), the time complexity is exponential. In this latter case, the number of conditional independence tests required by the IC/PC to learn a causal PGM with $p$ vertices and maximum degree (the largest number of edges incident to a vertex) $k$ is bounded above by $p^{k+2}$ (Spirtes and Meek, 1995).

The unrealistic assumption of causal sufficiency is relaxed by the FCI- (Fast Causal Inference) and RFCI- (Really Fast Causal Inference) algorithms and their variants (Colombo et al., 2012; Spirtes et al., 2000). These algorithms are nearly identical to the IC/PC algorithm. They employ an adjacency phase analogous to the IC/PC algorithm, but additional conditional independence tests are performed in the orientation phase to account for unmeasured common causes.

For learning Gaussian DAGs, a zero partial correlation test is used as d-separation oracle in all constraint-based algorithms. The estimator based on univariate linear regressions, derived in Section 2.3.1, can be used for computing the partial correlation coefficients give all possible conditioning sets $\boldsymbol{S}$. 


\subsection{Learning Gaussian PGMs from Family Data}

Family data consist of (multiple) traits along with suitable covariates from members of a large number of independent families. Observations from individuals of different families are supposed to be independent, but observations from members of a same family may be correlated, since they share a genetic component. In the context of univariate analysis, family data have been widely used to decompose trait variance in terms of genetic (commonly, the additive genetic component or polygenic) and error (in general, the environmental component) factors.

In this paper, we exploit family data to understand how the covariance among multiple traits is affected by genetic and environmental factors. Besides extending methods for learning PGMs from family data, we provide a decomposition of the graphical model into genetic and environmental components, showing the potential impact of these factors on the relationships among the traits.

In proband-oriented family-based design, first an individual (called proband) is sampled and then their respective family members (non-probands) are recruited for study. The proband may be randomly chosen from the population or selected according to eligibility or ascertainment criteria. For studying a specific trait or disease, families with large pedigrees and many affected (related) individuals are the most informative. Since it may be particularly difficult to obtain a large enough number of well-characterized families, several recruitment strategies are proposed. In family-based case-control studies, for example, the enrollment of families into the study is typically through affected probands. A case is typically ascertained, e.g. from a hospital based registry, and then his or her family is studied. However, this non-random selection may lead to an ascertainment bias. For learning the structure of PGMs, data should be free of ascertainment bias in order to avoid spurious findings of familial aggregation. Therefore, we assume data collected from randomly ascertained pedigrees, i.e., from families which were not selected for a specific trait or disease. If a non-random family sampling is performed, a correction for ascertainment bias must be applied (Bethony et al., 2001; Siegmund and Langholz, 2002; Ziogas, 1996).

\subsubsection{Confounding, Paradoxes, and Fallacies}

PGMs are a useful tool to identify variables that must be controlled for when studying an association of interest. Biases due to spurious associations may arise if confounders, mediators, and colliders are inappropriately modeled Sjölander and Zetterqvist (2017). Such biases can also occur when analyzing structured data, such as family data, if the grouping factor is not taken into account Friendly et al. (2013). Thus, to properly understand how the variables are related to each other in family-based studies, it is important to analyze the relationships partitioned according to the sources of variation. In other words, when dealing with family data, it is important to provide a decomposition of the learned PGM into 
between-family (genetic) and within-family (environmental) components to avoid fallacious interpretations and, consequently, false conclusions.

To exemplify this problem, we show in Figure 2.3 a fictitious causal PGM describing, in a very simplified way, the relationships among Exercise, Stress, and Depression. For simplicity, the variables are normally distributed and no other variable is important for this system, so the assumption of causal sufficiency is satisfied. In A), it is shown the total PGM, in which the causal effects are determined without decomposing the trait covariance matrix. In B) and C) are shown, respectively, the decomposition of the total graph into (betweenfamily) genetic and environmental (within-family) graphs. In plots D) and E) are shown, respectively for the marginal and partial correlations between Exercise and Stress, the 95\% concentration ellipses Dempster (1969) for both the between-family (around the intercepts of the families) and for the within-family covariance matrices.

From any causal model, we can read off total effects and their decomposition into direct and indirect effects between any pair of variables Pearl (2001). Direct effects are those not mediated by any other variable in the model. Indirect effects are those that go through mediators. And total effects are a combination of direct and indirect effects. In linear models, the total effect is the sum of both the direct and the indirect effects Bollen (1987).

When trying to assess the impact of a variable on the other (total or direct, depending on the research question), it is necessary to know precisely all connections among the variables and then to decide about whether or not conditioning on some variable. For the models represented in Graphs 2.3-A, B, and C, the direct effect between Exercise and Depression is given by blocking the indirect path through Stress, i.e, by conditioning on Stress. So, considering that all variables are Gaussian, its significance can be tested by testing the partial correlation between Exercise and Depression given Stress. However, the significance of the direct effect between Exercise and Stress has to be accessed by the marginal correlation between them, since the path through Depression is already blocked (Depression is a collider). In this case, the partial correlation between Exercise and Stress given Depression reflects the total effect from Exercise to Stress, since conditioning on Depression makes the path through it unblocked.

As shown in Plot (D) of Figure 2.3, both the within- and between-family marginal correlations (and so the direct effects) between Exercise and Stress are negative (according to the directions of the largest axis of all ellipses). However, as shown in Plot (E), the partial correlations between Exercise and Stress given Depression (and so the total effects) are completely different: the within-family partial correlation is still negative, but smaller, and the between-family partial correlation is now positive. Since the within- and between-family partial correlations between Exercise and Stress given Depression have opposite signs, that is a typical example of Robinson's paradox Robinson (2009) and any extrapolation from within-family conclusions to between-family conclusions, or vice-versa, would be a fallacious reasoning.

It's easy to understand what is happening in this example. It is common to think that 
Exercises and Stress are negatively correlated (the higher the level of physical activity, the lower the degree of stress). However, according to our fictitious example, conditioned on Depression (i.e., considering individuals with the same depression level), if the physical activity is high, then probably the degree of stress is also considerably high. In other words, an spurious positive correlation between Exercise and Stress (positive indirect effect) is created by conditioning on Depression. Since the total effect (whose sign and significance can be assessed by the partial correlation between Exercise and Stress given Depression) is computed by summing up the effects of all unblocked paths, it may be positive if that positive indirect effect is too strong, as happened in the genetic graph (B), or may be negative, but smaller, as happened in the environmental graph (C), or may be even close to zero, if the negative direct effect is canceled out by the positive indirect effect, as happened in the total graph (A).

Note that this example also illustrates a violation of the faithfulness assumption, since data are unfaithful to the causal model represented in the total graph (A). Specifically, the total effect between Exercise and Stress is considered zero in the total graph, since the partial correlation between Exercise and Stress given Depression is almost zero. That only happens because the indirect effect of the path through Depression cancel out the direct effect. Thus, a false conditional independence relation between Exercise and Stress does not allow the correct learning of the total graph. However, the partial correlation between Exercise and Stress given Depression in the total graph is decomposed into a positive partial correlation in the genetic graph and a negative partial correlation in the environmental graph. Thus, the faithfulness assumption holds for the genetic and environmental graphs, allowing their structures to be reliably learned.

\subsubsection{Partial Correlation for Family Data}

The same ideas used for learning PGMs from independent observational units can be exploited for learning PGMs from family data. However, since classical conditional independence tests are valid only under the assumption of independent observations, we have to use a proper zero partial correlation test that accounts for the familial dependence structure.

Consider a sample of $N$ individuals organized in $F$ families, each containing $n_{f}$ members $(f=1, \ldots, F)$. The total number of individuals is $N=\sum_{f=1}^{F} n_{f}$. Let $\boldsymbol{V}$ be a random vector of multiple traits following a multivariate normal distribution. Also, let $X, Y \in \boldsymbol{V}$ and $\boldsymbol{S} \subseteq \boldsymbol{V} \backslash\{X, Y\}$. Considering the partial correlation estimator for independent data, shown in Section 2.3.1, we propose an extension for family data by fitting univariate polygenic linear mixed models with two components of variance (Almasy and Blangero, 1998; Amos, 1994; Lange, 2003).

Consider that the observations of $X$ and $Y$ for individual $i$ of family $f$ can be expressed, 
(A) Total Graph

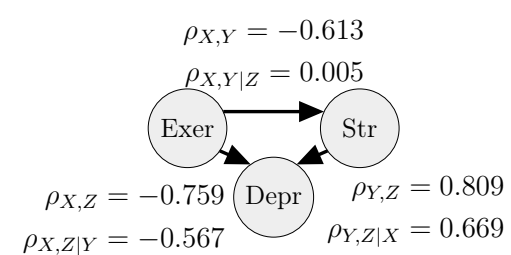

(B) Genetic Graph

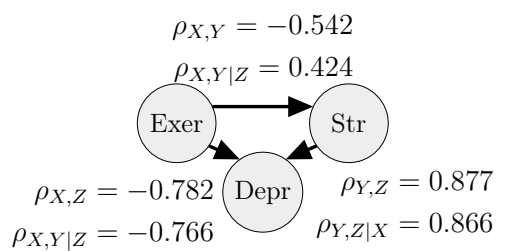

(C) Environmental Graph

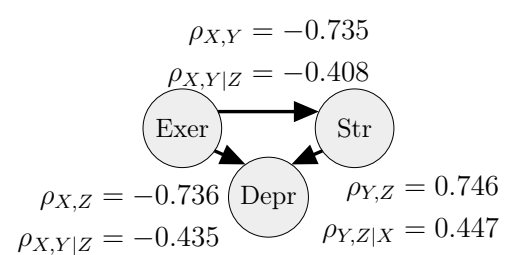

(D)

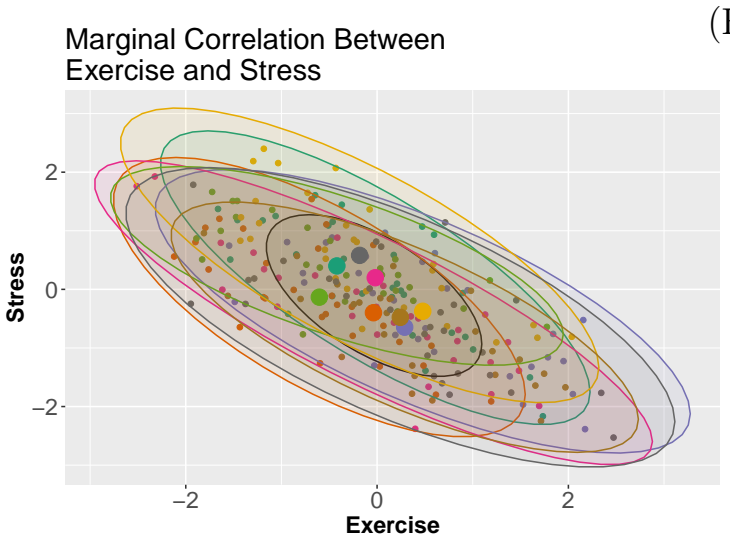

$(\mathrm{E})$

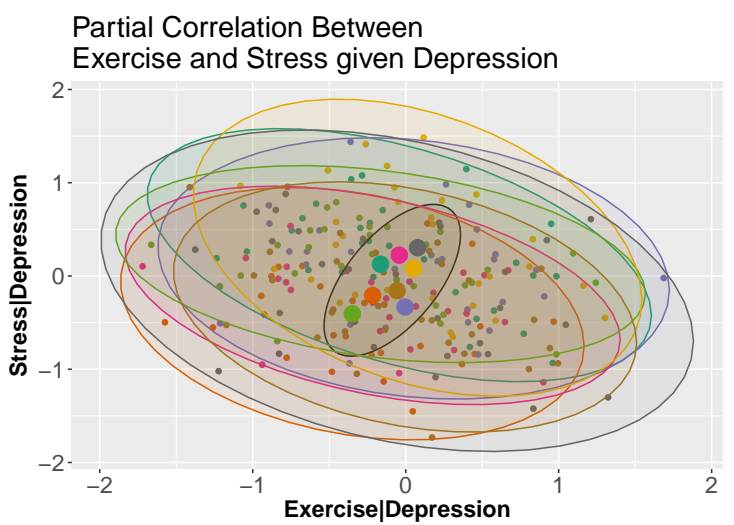

Figure 2.3: Fictitious causal relationships among Exercise (Exer), Stress (Str), and Depression (Depr), graphically represented by three PGMs: (A) total (determined without decomposing the trait covariance matrix), (B) genetic (between-family), and (C) environmental (within-family) graphs. The marginal and partial correlation coefficients between the variables $X$ (Exer), $Y$ (Str), and $Z$ (Depr) are shown adjacent to the edges. Plots (D) and (E) show the association between Exercise and Stress marginally and conditioned on Depression, respectively. The concentration ellipses around the intercepts of the families (the big dots) represent the genetic (between-family) covariance matrix, while the other concentration ellipses show the environmental (within-family) covariance matrix. 
respectively, as:

$$
\begin{gathered}
x_{i f}=\alpha^{X(\boldsymbol{S})}+\sum_{k=1}^{|\mathbf{S}|} \beta_{k}^{X(\boldsymbol{S})} s_{k i f}+\gamma_{i f}^{X(\boldsymbol{S})}+\epsilon_{i f}^{X(\boldsymbol{S})}, \\
y_{i f}=\alpha^{Y(\boldsymbol{S})}+\sum_{k=1}^{|\mathbf{S}|} \beta_{k}^{Y(\boldsymbol{S})} s_{k i f}+\gamma_{i f}^{Y(\boldsymbol{S})}+\epsilon_{i f}^{Y(\boldsymbol{S})},
\end{gathered}
$$

where, in the modeling for variable X, $\alpha^{X(\boldsymbol{S})}$ is the intercept, $\beta_{k}^{X(\boldsymbol{S})}$ and $s_{k i}$, for $k=1, \ldots,|\boldsymbol{S}|$, are, respectively, the parameters and the observed values of the fixed effects of the variables in $\boldsymbol{S}$, and $\gamma_{i f}^{X(\boldsymbol{S})}$ and $\epsilon_{i f}^{X(\boldsymbol{S})}$ are mutually independent and normally distributed random effects, with zero mean and, respectively, variance $\sigma_{g_{X \mid S}}^{2}$ and $\sigma_{e_{X \mid S}}^{2}$. The random effects $\gamma_{i f}^{X(\boldsymbol{S})}$ and $\epsilon_{i f}^{X(S)}$ mainly model the variability due to, respectively, between-family (genetic) and withinfamily (environmental) factors. The notation is analogous in the modeling for $Y$.

Considering no inbreeding families, the information on the dependence among individuals in each family $f=1, \ldots, F$ is provided by the $n_{f} \times n_{f}$ kinship matrix $\boldsymbol{\Phi}^{(f)}$, in which each entry $\Phi_{i j}^{(f)}$ represents the probability that two alleles sampled at random from each individual are identical by descent. When the pedigree structure is known, the kinship matrix is computed according to the degree of kinship between individuals: $\Phi_{i i}^{(f)}=1 / 2, \Phi_{i j}^{(f)}=1 / 4$ if $i$ and $j$ are siblings or if one of them is a parent of the other, $\Phi_{i j}^{(f)}=1 / 8$ if one of them is a grand-parent of the other, and so on. Thus, the covariance between response variables for individuals $i$ and $j$ of, respectively, families $f$ and $f^{\prime}$, considering variable $\mathrm{X}$, is as follows:

$$
\operatorname{Cov}\left(x_{i f}, x_{j f^{\prime}}\right)= \begin{cases}\sigma_{g_{X \mid S}}^{2}+\sigma_{e_{X \mid S}}^{2}, & \text { if } i=j ; f=f^{\prime} \\ 2 \Phi_{i j}^{(f)} \sigma_{g_{X \mid S}}^{2}, & \text { if } i \neq j ; f=f^{\prime} \\ 0, & \text { if } i \neq j ; f \neq f^{\prime} .\end{cases}
$$

To model the underlying dependence structure among the individuals of a same family $f$, consider a $n_{f} \times n_{f}$ design matrix $\boldsymbol{Z}_{f}$ for the random effects, such that $\boldsymbol{Z}_{f} \boldsymbol{Z}_{f}^{\prime}=2 \boldsymbol{\Phi}^{(f)}$. Let $\gamma_{f}^{X(\boldsymbol{S})}=\left(\gamma_{1 f}^{X(\boldsymbol{S})}, \ldots, \gamma_{n_{f} f}^{X(\boldsymbol{S})}\right)^{\prime}$. Then, $\boldsymbol{g}_{f}^{X(\boldsymbol{S})}=\boldsymbol{Z}_{f} \boldsymbol{\gamma}_{f}^{X(\boldsymbol{S})} \sim \mathcal{N}_{n_{f}}\left(\mathbb{1}_{n_{f}} \otimes 0,2 \boldsymbol{\Phi}^{(f)} \sigma_{g_{X \mid \boldsymbol{S}}}^{2}\right)$ and $\boldsymbol{\epsilon}_{f}^{X(\boldsymbol{S})}=\left(\boldsymbol{\epsilon}_{1 f}^{X(\boldsymbol{S})}, \ldots, \boldsymbol{\epsilon}_{n_{f} f}^{X(\boldsymbol{S})}\right)^{\prime} \sim \mathcal{N}_{n_{f}}\left(\mathbb{1}_{n_{f}} \otimes 0, \boldsymbol{I}_{n_{f}} \sigma_{e_{X \mid S}}^{2}\right)$ are the $n_{f}$-dimensional vectors of, respectively, genetic and environmental random-effects.

To represent the $N$-dimensional vector $\boldsymbol{x}$, with measurements of $X$ for all individuals, let $\boldsymbol{\gamma}^{X(\boldsymbol{S})}=\left(\boldsymbol{\gamma}_{1}^{X(\boldsymbol{S})}, \ldots, \boldsymbol{\gamma}_{F}^{X(\boldsymbol{S})}\right)^{\prime}, \boldsymbol{Z}=\bigoplus_{f=1}^{F} \boldsymbol{Z}_{i}$, and $\boldsymbol{\Phi}=\bigoplus_{f=1}^{F} \boldsymbol{\Phi}^{(f)}$, where $\bigoplus$ indicates the direct sum (Searle and Khuri, 2017). Also, let $\boldsymbol{D}^{X(\boldsymbol{S})}=\boldsymbol{I}_{N} \sigma_{g_{X \mid \boldsymbol{S}}}^{2}$ and $\boldsymbol{R}^{X(\boldsymbol{S})}=$ $\boldsymbol{I}_{N} \sigma_{e_{X \mid S}}^{2}$. Then $\boldsymbol{g}^{X(\boldsymbol{S})}=\left(\boldsymbol{g}_{1}^{X(\boldsymbol{S})}, \ldots, \boldsymbol{g}_{F}^{X(\boldsymbol{S})}\right)^{\prime}=\boldsymbol{Z} \boldsymbol{\gamma}^{X(\boldsymbol{S})} \sim \mathcal{N}_{N}\left(\mathbb{1}_{N} \otimes 0, \mathbf{2} \boldsymbol{\Phi} \sigma_{g_{X \mid S}}^{2}\right)$ and $\boldsymbol{\epsilon}^{X(\boldsymbol{S})}=$ $\left(\boldsymbol{\epsilon}_{1}^{X(\boldsymbol{S})}, \ldots, \boldsymbol{\epsilon}_{F}^{X(\boldsymbol{S})}\right)^{\prime} \sim \mathcal{N}_{N}\left(\mathbb{1}_{N} \otimes 0, \boldsymbol{I}_{N} \sigma_{e_{X \mid S}}^{2}\right)$. Let $\boldsymbol{X}_{\boldsymbol{S}}$ be the $N \times|\boldsymbol{S}|$ design matrix and $\boldsymbol{\beta}^{X(\boldsymbol{S})}$ be the $|\boldsymbol{S}| \times 1$ vector of parameters for the fixed effects of the variables in $\boldsymbol{S}$.

The matrix representation of the polygenic linear mixed model for $\boldsymbol{x}$, accounting for the 
variables in $\boldsymbol{S}$, is given by

$$
\boldsymbol{x}=\boldsymbol{X}_{\boldsymbol{S}} \boldsymbol{\beta}^{X(\boldsymbol{S})}+\boldsymbol{Z} \boldsymbol{\gamma}^{X(\boldsymbol{S})}+\boldsymbol{\epsilon}^{X(\boldsymbol{S})},
$$

and the $N \times N$ covariance matrix of $\boldsymbol{x}$ is given by

$$
\begin{aligned}
\operatorname{Cov}\left(\boldsymbol{g}^{X(\boldsymbol{S})}+\boldsymbol{\epsilon}^{X(\boldsymbol{S})}\right) & =\boldsymbol{Z} \boldsymbol{D}^{X(\boldsymbol{S})} \boldsymbol{Z}^{\prime}+\boldsymbol{R}^{X(\boldsymbol{S})} \\
& =2 \boldsymbol{\Phi} \sigma_{g_{X \mid S}}^{2}+\boldsymbol{I}_{N} \sigma_{e_{X \mid \boldsymbol{S}}}^{2}
\end{aligned}
$$

Analogous notations are used for specifying the covariance structure among measurements for $Y$.

Considering that the (co)variance is decomposed into two components, three partial correlation coefficients between $X$ and $Y$ given $\boldsymbol{S}$ can be estimated: i) a genetic or betweenfamily coefficient, obtained by the Pearson correlation between the predicted random-effects $\hat{\boldsymbol{g}}^{X(\boldsymbol{S})}$ and $\hat{\boldsymbol{g}}^{Y \mid \boldsymbol{S}}$; ii) an environmental or within-family coefficient, obtained by the Pearson correlation between the conditional residuals $\hat{\boldsymbol{\epsilon}}^{X(\boldsymbol{S})}$ and $\hat{\boldsymbol{\epsilon}}^{Y \mid \boldsymbol{S}}$; and iii) a total coefficient, obtained by the Pearson correlation between the marginal residuals, $\hat{\boldsymbol{g}}^{X(\boldsymbol{S})}+\hat{\boldsymbol{\epsilon}}^{X(\boldsymbol{S})}$ and $\hat{\boldsymbol{g}}^{Y \mid \boldsymbol{S}}+\hat{\boldsymbol{\epsilon}}^{Y \mid S}$.

\subsubsection{Confounded Residuals and Predicted Random Effects}

Pure residuals should only depend on the error that it is supposed to predict. However, as noted by Hilden-Minton (1995), when obtained by maximum likelihood (ML) or restricted maximum likelihood (REML) estimators, the conditional residuals $\left(\hat{\boldsymbol{\epsilon}}^{X(\boldsymbol{S})}\right.$ or $\left.\hat{\boldsymbol{\epsilon}}^{Y(\boldsymbol{S})}\right)$ are confounded with their respective random effects $\left(\boldsymbol{g}^{X(\boldsymbol{S})}\right.$ or $\left.\boldsymbol{g}^{Y(\boldsymbol{S})}\right)$ and the predicted random effects $\left(\hat{\boldsymbol{g}}^{X(\boldsymbol{S})}\right.$ or $\left.\hat{\boldsymbol{g}}^{Y(\boldsymbol{S})}\right)$ are confounded with their respective conditional errors $\left(\boldsymbol{\epsilon}^{X(\boldsymbol{S})}\right.$ or $\left.\boldsymbol{\epsilon}^{Y(\boldsymbol{S})}\right)$. Thus, the partial correlation coefficients will also be confounded if estimated from these residuals as they are.

Inspired by Loy and Hofmann (2015), we developed a strategy to reduce this confounding by determining for each residual (in each model, for $\mathrm{X}$ and $\mathrm{Y}$ ) a low-dimensional approximation that minimizes the variability of the error that it is not supposed to predict at the same time that maximizes the total variability.

Denote the $N \times N$ inverse covariance matrix of $\boldsymbol{x}$, in Equation 2.2, by $\boldsymbol{M}^{X(\boldsymbol{S})}=$ $\left(\boldsymbol{Z} \boldsymbol{D}^{X(\boldsymbol{S})} \boldsymbol{Z}^{\prime}+\boldsymbol{R}^{X(\boldsymbol{S})}\right)^{-1}$. Also, let:

$$
\boldsymbol{Q}^{X(\boldsymbol{S})}=\boldsymbol{M}^{X(\boldsymbol{S})}-\boldsymbol{M}^{X \mid \boldsymbol{S}} \boldsymbol{X}_{\boldsymbol{S}}\left(\boldsymbol{X}_{\boldsymbol{S}}^{\prime} \boldsymbol{M}^{X(\boldsymbol{S})} \boldsymbol{X}_{\boldsymbol{S}}\right)^{-1} \boldsymbol{X}_{\boldsymbol{S}} \boldsymbol{M}^{X(\boldsymbol{S})}
$$

The ML (or REML) predicted genetic random-effects and environmental residuals are given, respectively, by:

$$
\begin{gathered}
\hat{\boldsymbol{g}}^{X(\boldsymbol{S})}=\boldsymbol{Z} \boldsymbol{D}^{X(\boldsymbol{S})} \boldsymbol{Z}^{\prime} \boldsymbol{Q}^{X(\boldsymbol{S})} \boldsymbol{x} \\
\hat{\boldsymbol{\epsilon}}^{X(\boldsymbol{S})}=\boldsymbol{R}^{X(\boldsymbol{S})} \boldsymbol{Q}^{X(\boldsymbol{S})} \boldsymbol{x} .
\end{gathered}
$$


Consider that $\boldsymbol{Q}^{X(\boldsymbol{S})}, \boldsymbol{R}^{X(\boldsymbol{S})}$, and $\boldsymbol{D}^{X(\boldsymbol{S})}$ are symmetric and positive definite matrices. Also, note that $\boldsymbol{Q}^{X(\boldsymbol{S})}\left(\boldsymbol{M}^{X(\boldsymbol{S})}\right)^{-1} \boldsymbol{Q}^{X(\boldsymbol{S})}=\boldsymbol{Q}^{X(\boldsymbol{S})}$. Then, the $N \times N$ covariance matrix of $\hat{\boldsymbol{g}}^{X(\boldsymbol{S})}$ is given by

$$
\boldsymbol{\Sigma}_{\hat{\boldsymbol{g}}^{X(S)}}=\boldsymbol{Z} \boldsymbol{D}^{X(\boldsymbol{S})} \boldsymbol{Z}^{\prime} \boldsymbol{Q}^{X(\boldsymbol{S})} \boldsymbol{Z} \boldsymbol{D}^{X(\boldsymbol{S})} \boldsymbol{Z}^{\prime}
$$

but the following part is due to the conditional errors:

$$
\boldsymbol{\Sigma}_{\hat{\boldsymbol{g}}^{X(\boldsymbol{S})} \mid \boldsymbol{g}^{X(\boldsymbol{S})}}=\boldsymbol{Z} \boldsymbol{D}^{X(\boldsymbol{S})} \boldsymbol{Z}^{\prime} \boldsymbol{Q}^{X(\boldsymbol{S})} \boldsymbol{R}^{X(\boldsymbol{S})} \boldsymbol{Q}^{X(\boldsymbol{S})} \boldsymbol{Z} \boldsymbol{D}^{X(\boldsymbol{S})} \boldsymbol{Z}^{\prime}
$$

Similarly, the $N \times N$ covariance matrix of $\hat{\boldsymbol{\epsilon}}^{X(\boldsymbol{S})}$ is given by

$$
\boldsymbol{\Sigma}_{\hat{\boldsymbol{\epsilon}}^{X(\boldsymbol{S})}}=\boldsymbol{R}^{X(\boldsymbol{S})} \boldsymbol{Q}^{X(\boldsymbol{S})} \boldsymbol{R}^{X(\boldsymbol{S})}
$$

but the following part is due to the random effects:

$$
\boldsymbol{\Sigma}_{\hat{\boldsymbol{\epsilon}}^{X(\boldsymbol{S})} \mid \boldsymbol{\epsilon}^{X(\boldsymbol{S})}}=\boldsymbol{R}^{X(\boldsymbol{S})} \boldsymbol{Q}^{X(\boldsymbol{S})} \boldsymbol{Z} \boldsymbol{D}^{X(\boldsymbol{S})} \boldsymbol{Z}^{\prime} \boldsymbol{Q}^{X(\boldsymbol{S})} \boldsymbol{R}^{X(\boldsymbol{S})}
$$

Consider the analogous definitions for the covariance matrices of $\hat{\boldsymbol{g}}^{Y \mid \boldsymbol{S}}$ and $\hat{\boldsymbol{\epsilon}}^{Y \mid \boldsymbol{S}}$.

For a given dimension, denoted by $s_{g}$, consider the rotation matrix $\boldsymbol{W}_{\boldsymbol{g}}^{*}\left(s_{g}\right)$ obtained so that the fraction of confounding is minimal for the predicted genetic random effects of both $X$ and $Y$, i.e, obtained by solving the following minimization problem:

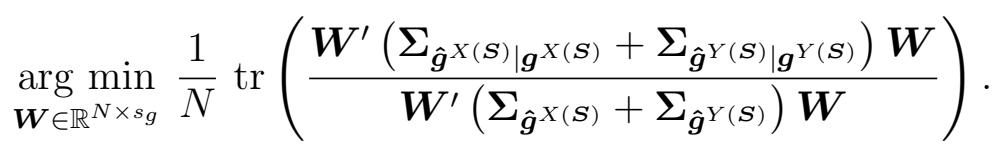

Analogously, for a given dimension, denoted by $s_{e}$, consider the rotation matrix $\boldsymbol{W}_{\boldsymbol{\epsilon}}^{*}\left(s_{e}\right)$ obtained so that the fraction of confounding is minimal for the conditional (environmental) residuals of both $X$ and $Y$, i.e, by solving the following minimization problem:

$$
\underset{\boldsymbol{W} \in \mathbb{R}^{N \times s_{e}}}{\arg \min } \frac{1}{N} \operatorname{tr}\left(\frac{\boldsymbol{W}^{\prime}\left(\boldsymbol{\Sigma}_{\hat{\boldsymbol{\epsilon}}^{X(S)} \mid \boldsymbol{\epsilon}^{X(\boldsymbol{S})}}+\boldsymbol{\Sigma}_{\hat{\boldsymbol{\epsilon}}^{Y(\boldsymbol{S})} \mid \boldsymbol{\epsilon}^{Y(S)}}\right) \boldsymbol{W}}{\boldsymbol{W}^{\prime}\left(\boldsymbol{\Sigma}_{\hat{\boldsymbol{\epsilon}}^{X(S)}}+\boldsymbol{\Sigma}_{\hat{\boldsymbol{\epsilon}}^{Y(S)}}\right) \boldsymbol{W}}\right) .
$$

The objective functions of these minimization problems are generalized Rayleigh quotients (Parlett, 1998), whose minimum can be found through a generalized eigenvalue decomposition of the matrices. Thus, the columns of $\boldsymbol{W}_{\boldsymbol{g}}^{*}\left(s_{g}\right)$ are the $s_{g}$ eigenvectors of the matrix $\left(\boldsymbol{\Sigma}_{\hat{\boldsymbol{g}}^{X(\boldsymbol{S})}}+\boldsymbol{\Sigma}_{\hat{\boldsymbol{g}}^{Y(\boldsymbol{S})}}\right)^{-1}\left(\boldsymbol{\Sigma}_{\hat{\boldsymbol{g}}^{X(\boldsymbol{S})} \mid \boldsymbol{g}^{X(\boldsymbol{S})}}+\boldsymbol{\Sigma}_{\hat{\boldsymbol{g}}^{Y(\boldsymbol{S})} \mid \boldsymbol{g}^{Y(\boldsymbol{S})}}\right)$ corresponding to the $s_{g}$ smallest eigenvalues. The same idea is used to determine $\boldsymbol{W}_{\boldsymbol{e}}^{*}\left(s_{e}\right)$.

The unconfounded genetic partial correlation between $X$ and $Y$ given $\boldsymbol{S}$ can be estimated by the Pearson correlation between $\boldsymbol{W}_{\hat{\boldsymbol{g}}}^{*}\left(s_{g}\right)^{\prime} \hat{\boldsymbol{g}}^{X(\boldsymbol{S})}$ and $\boldsymbol{W}_{\hat{\boldsymbol{g}}}^{*}\left(s_{g}\right)^{\prime} \hat{\boldsymbol{g}}^{Y(\boldsymbol{S})}$, and the unconfounded environmental partial correlation can be estimated by the Pearson correlation between $\boldsymbol{W}_{\hat{\epsilon}}^{*}\left(s_{e}\right)^{\prime} \hat{\boldsymbol{\epsilon}}^{\boldsymbol{X}}$ and $\boldsymbol{W}_{\hat{\epsilon}}^{*}\left(s_{e}\right)^{\prime} \hat{\boldsymbol{\epsilon}}^{\boldsymbol{Y}}$.

The choice of the dimensions $s_{g}$ and $s_{e}$ in these dimensionality reduction problems is 
critical, since it is based on a trade-off between statistical power and reliability. We have to reduce dimension for reducing confounding, but we cannot reduce too much because we will lose statistical power.

Thus, we propose an algorithm for selecting the best dimension, based on two inputs: the maximum fraction of confounding and the minimum dimension that are acceptable. Given a significance level, the algorithm first tests whether the total partial correlation coefficient is zero. If so, then either both, the genetic and the environmental partial correlation estimates are zero, or they have opposite signs. If not, then the genetic or the environmental partial correlation coefficient (or both) should be non-zero. The algorithm tries to infer which is the case and selects the best dimensions $s_{g}$ and $s_{e}$, giving preference to those where the respective estimates have stabilized stabilized around some value (i.e., after convergence has been reached).

When observations are correlated, as in family data, the effective sample size is the number of independent units in a sample that is relevant to estimate a parameter. In these minimization problems, the values $s_{g}$ and $s_{e}$ are the number of independent units required to estimate, respectively, the unconfounded genetic and environmental partial correlations. So they can be understood as effective sample sizes for, respectively, the genetic and the environmental components, and can be used in statistical tests to avoid overestimation of the p-values. Thus, to test the null hypotheses of zero partial correlation in the genetic and environmental components, we can conduct the usual Fisher's z test (see Section 2.3.1) directly on the respective unconfounded sample partial correlation coefficient, which has the correct sample size $\left(s_{g}\right.$ or $\left.s_{e}\right)$.

\subsubsection{Structure Learning from Gaussian Family Data}

Let $\boldsymbol{V}$ be a $p$-dimensional Gaussian vector with mean $\boldsymbol{\mu}_{p}$ and covariance matrix $\boldsymbol{\Sigma}_{p \times p}$, which can be decomposed into genetic and an environmental covariance matrices, denoted, respectively, by $\Sigma_{g_{p \times p}}$ and $\Sigma_{e_{p \times p}}$. Also, consider the $N \times p$ data matrix with $N$ observations for $\boldsymbol{V}$, grouped by families, and its vectorization, $\boldsymbol{v}$, obtained by stacking its rows. The dependence among all individuals is provided by the relatedness matrix $2 \boldsymbol{\Phi}$. Inspired by the structure learning algorithms described in Section 2.3, we developed algorithms for learning the structure of an undirected or a directed Gaussian PGM that perfectly encodes the multivariate Gaussian distribution

$$
\boldsymbol{v} \sim \mathcal{N}_{N p}\left(\mathbb{1}_{N} \otimes \boldsymbol{\mu}_{p}, 2 \Phi \otimes \boldsymbol{\Sigma}_{g_{p \times p}}+\mathbf{I}_{N} \otimes \boldsymbol{\Sigma}_{e_{p \times p}}\right)
$$

based on the unconfounded partial correlation tests proposed in Section 2.4.2. Since they are based on univariate polygenic linear mixed models, in which the random part is decomposed into a genetic and an environmental component, the algorithms also provide a decomposition of the PGM into the same components. Thus, three different PGMs can be learned: the total, 
the genetic, and the environmental PGM.

For learning undirected Gaussian PGMs, a standard approach is to assign an edge between every pair of variables such that the partial correlation given all the other variables is significantly different from zero. From observational family data, we propose to make these decisions by using the zero partial correlation tests derived from univariate polygenic linear mixed models. Thus, they can be made separately for each component. Two variables are separated in the genetic PGM only if the unconfounded genetic partial correlation between them given the other variables is zero. Analogously, the same two variables are separated in the environmental PGM only if the unconfounded environmental partial correlation between them given the other variables is zero. Finally, they are separated in the total PGM if the partial correlation between them given the other variables, obtained from the marginal residuals, is zero.

The IC/PC algorithm is the standard algorithm for learning directed acyclic PGMs. Several conditional independence tests are performed for each pair of variables in a search for a conditioning set that makes the pair conditionally independent. For learning from Gaussian family data, we propose to use as d-separation oracles the zero partial correlation tests derived from univariate polygenic linear mixed models. Thus, the search can be performed separately for the genetic, environmental, and total components. If there exists a conditioning set such that the unconfounded genetic partial correlation between the two variables is zero, then the pair is d-separated in the genetic PGM. Analogously, if there exists a conditioning set such that the unconfounded environmental partial correlation between the two variables is zero, then the pair is d-separated in the environmental PGM. Finally, if there exists a conditioning set such that the partial correlation obtained from the marginal residuals is zero, then the pair is d-separated in the total PGM. At the end of this step, the orientation step is then applied to the skeleton of each component to recover the respective CPDAGs.

\subsubsection{FamilyBasedPGMs R Package}

The proposed methods for learning undirected and directed PGM from family data are available at the FamilyBasedPGMs R package on GitHub at https://github.com/adele/ FamilyBasedPGMs. The PC algorithm is implemented using the pcalg R package (Kalisch et al., 2012), and the univariate polygenic mixed models are estimated using the function lmekin of the coxme $R$ package (Therneau, 2012).

Considering that the proposed algorithm for leaning undirected PGMs runs in quadratic time (as a function of the number of vertices) and that the IC/PC algorithm runs in exponential time for non-sparse PGMs (the worst case scenario), we implemented, for all methods, pre-processing functions that compute all necessary zero partial correlation tests in parallel on the available CPU cores. 


\subsection{Simulation Study}

First, we simulated different pedigrees for 30 families, each with 30 individuals. The same pedigrees were used for simulating 100 replicates of family data for three Gaussian phenotypes, namely $X, Y$, and $Z$. The familial dependence structure, represented by the $2 \Phi$ matrix, was used to generate correlated observations within families. The relationships among these three phenotypes was determined by the following recursive SEM with genetic and environmental variance components:

$$
\left\{\begin{array}{l}
\boldsymbol{x}=\boldsymbol{g}^{X}+\boldsymbol{\epsilon}^{X} \\
\boldsymbol{y}=\boldsymbol{\beta}_{y x} \boldsymbol{x}+\boldsymbol{g}^{Y(X)}+\boldsymbol{\epsilon}^{Y(X)} \\
\boldsymbol{z}=\boldsymbol{\beta}_{z x} \boldsymbol{x}+\boldsymbol{\beta}_{z y} \boldsymbol{y}+\boldsymbol{g}^{Z(X ; Y)}+\boldsymbol{\epsilon}^{Z(X ; Y)}
\end{array}\right.
$$

where $\boldsymbol{g}^{X} \sim \mathcal{N}_{N}\left(0,2 \boldsymbol{\Phi} \sigma_{g_{X}}^{2}\right)$ and $\boldsymbol{\epsilon}^{X} \sim \mathcal{N}_{N}\left(0, \boldsymbol{I}_{N} \sigma_{e_{X}}^{2}\right)$ are, respectively, the polygenic random effect (denoted as genetic component) and the error term (denoted as the environment component) for the variable $X ; \boldsymbol{g}^{Y(X)} \sim \mathcal{N}_{N}\left(0,2 \boldsymbol{\Phi} \sigma_{g_{Y \mid X}}^{2}\right)$ and $\boldsymbol{\epsilon}^{Y(X)} \sim \mathcal{N}_{N}\left(0, \boldsymbol{I}_{N} \sigma_{e_{Y \mid X}}^{2}\right)$ are, respectively, the genetic random effect and the environmental error term for the variable $Y$, after removing the fixed effect of the variable $X$; and $\boldsymbol{g}^{Z(X ; Y)} \sim \mathcal{N}_{N}\left(0,2 \boldsymbol{\Phi} \sigma_{g_{Z \mid\{X, Y\}}}^{2}\right)$ and $\boldsymbol{\epsilon}^{Z(X ; Y)} \sim \mathcal{N}_{N}\left(0, \boldsymbol{I}_{N} \sigma_{e_{Z \mid\{X, Y\}}}^{2}\right)$ are, respectively, the genetic random effect and the error term for the variable $Z$, after removing the contribution of the variables $X$ and $Y$.

Note that all the genetic random effects and the error terms are independent of each other, implying that any correlation among the variables is due to their direct or indirect effects. Also, since no other variable is relevant to the system, the causal sufficiency assumption holds in the simulated datasets.

The marginal and partial correlations among the variables, depend on the coefficients $\boldsymbol{\beta}_{y x}, \boldsymbol{\beta}_{z x}$, and $\boldsymbol{\beta}_{z y}$ and also on the variances, which are decomposed into genetic and environmental components (Duarte et al., 2014). Thus, the contribution of genetic and environmental factors on the phenotypic covariance is controlled by the values of the variances $\sigma_{g_{X}}^{2}, \sigma_{e_{X}}^{2}, \sigma_{g_{Y \mid X}}^{2}, \sigma_{e_{Y \mid X}}^{2}, \sigma_{g_{Z \mid\{X, Y\}}}^{2}$, and $\sigma_{e_{Z \mid\{X, Y\}}}^{2}$. We considered four different scenarios, obtained by setting the SEM parameters as shown in Table 2.1 .

First, third, and fifth columns of Table 2.2 show, respectively for the total, genetic, and environmental PGMs, the adjacency matrices for the true DAGs and for the respective moral graphs in the four scenarios considered. Note that the moral graph is the equivalent undirected form of the DAG, since it contains only (but not necessarily all) conditional independence relations implied by the DAG.

The rows and columns of the adjacency matrices correspond to the variables $X, Y$, and $Z$, in that order. The entry in row $i$ and column $j$ is 1 when the variable $j$ causes the variable $i$ and is 0 otherwise. The true partial correlation coefficients in the total, genetic, and environmental PGMs are shown, respectively, in columns first, third, and fifth of Table 2.3 . 
The total, genetic, and environmental CPDAGs and moral graphs were recovered by applying the proposed methods for learning, respectively, directed and undirected Gaussian PGMs from family data, as described in Section 2.4.3. Since we are dealing with multiple statistical tests, it was used a significance level of 0.005 . Also, to minimize confounding in the genetic and environmental residuals, it was performed a dimensionality reduction so that the minimum dimension is 10 and the maximum fraction of confounding is $5 \%$.

\subsubsection{Simulation Results}

The simulation results for the total, genetic, and environmental PGMs in the four scenarios are shown, respectively, in the second, fourth, and sixth columns of Table 2.2. Since the CPDAG contains directed and undirected edges, the entries of the estimated adjacency matrix are the percentage of directed edges, followed by the percentage of undirected edges. The percentage of undirected edges is duplicated both above and below the main diagonal of the matrix.

The estimated partial correlation coefficients in the total, genetic, and environmental PGMs are shown, respectively, in the second, fourth, and sixth columns of Table 2.3. Note that the estimated genetic and environmental partial correlation coefficients are approximations of the true values, obtained by low dimensional approximations of the residuals in which part of the information was discarded to minimize the confounding between the genetic and environmental components. Thus, it is not expected that those estimates are not biased. We only expect that, even in the scenarios with problematic confounding, inferences on the association structure can be correctly drawn.

In scenario 1, only $\boldsymbol{\beta}_{y x}$ is zero, so, in the three PGMs, the true DAG is the unshielded collider $X \rightarrow Z \leftarrow Y$, and its moral graph is complete. In scenario 2, only $\boldsymbol{\beta}_{z y}$ is zero, so in the three PGMs the true DAG is the fork $Y \leftarrow X \rightarrow Z$ and its moral graph is exactly its undirected version. For these two first scenarios, there is a small number of false positive and false negative edge detections. The confounding in the conditional residuals and in the predicted random effects is not critical, since each edge has an effect of same sign in the three PGMs and moderate to high magnitude.

Scenario 3 is the situation shown in Figure 2.3, with $X, Y$, and $Z$ representing, respectively, the variables Exercise, Stress, and Depression. The edge $X \rightarrow Y$ is the most problematic, since $\rho_{X, Y \mid Z}$ have opposite signs in the genetic and environmental PGMs and is zero in the total PGM. In this situation, any confounding between the genetic and environmental residuals may lead to a wrong decision about the null hypothesis of zero partial correlation. On the other hand, a strong dimensionality reduction to minimize confounding may decreases too much the power to detect the edge. By using our approach for selecting the best dimension in the confounding correction, we still could recover this edge in $60 \%$ of cases in the genetic PGM and in $88 \%$ in the environmental PGM.

In the total and environmental PGMs of scenario 4, the true DAG is the unshielded 
Table 2.1: Values of the parameters of the causal model (2.3) used to generate the simulated data for scenarios 1 to 4 .

\begin{tabular}{lcccc}
\hline \hline Parameter & Scen. 1 & Scen. 2 & Scen. 3 & Scen. 4 \\
\hline $\boldsymbol{\beta}_{y x}$ & 0.000 & -0.800 & -0.800 & 0.000 \\
$\boldsymbol{\beta}_{z x}$ & 0.750 & 0.800 & -0.900 & 0.250 \\
$\boldsymbol{\beta}_{z y}$ & 0.800 & 0.000 & 0.900 & 0.550 \\
$\sigma_{X}^{2}$ & 0.875 & 0.425 & 0.600 & 0.000 \\
$\sigma_{X}^{2}$ & 0.250 & 0.600 & 0.550 & 2.500 \\
$\sigma_{Y \mid X}^{2}$ & 0.425 & 0.250 & 0.925 & 1.750 \\
$\sigma_{Y \mid X}^{2}$ & 0.625 & 0.725 & 0.300 & 0.750 \\
$\sigma_{Z \mid\{X, Y\}_{g}}^{2}$ & 0.250 & 0.475 & 0.250 & 0.750 \\
$\sigma_{Z \mid\{X, Y\}_{e}}^{2}$ & 0.275 & 0.275 & 0.975 & 0.250 \\
\hline \hline
\end{tabular}

collider $X \rightarrow Z \leftarrow Y$. However, the edge $X \rightarrow Z$ is absent in the genetic PGM, since, as shown in Table 2.3, the genetic part of both $\rho_{X, Z}$ and $\rho_{X, Z \mid Y}$ is zero. Considering that difference in the genetic and environmental structures, any confounding hinders the detection of the edge $X \rightarrow Z$. After correcting for confounding, we notice a relevant loss of statistical power for recovering the environmental structure. The method wrongly decided for $11 \%$ of cases that $\rho_{X, Z \mid Y}=0$ and for $15 \%$ of cases that $\rho_{X, Z}=0$. In the IC/PC algorithm, a decision affects all the subsequent ones. When inferring the skeleton, the edge between $X$ and $Z$ was removed in $18 \%$ of cases because it was determined that $X$ and $Z$ are either marginally independent or conditionally independent given $Y$. To orient the path connecting $X$ to $Y$ through $Z$ as an unshielded collider, $\rho_{X, Y \mid Z}$ must be non-zero and $\rho_{X, Y}$ must be zero. Since $\rho_{X, Y \mid Z}$ was considered zero in $1 \%$ of cases, the true DAG could be correctly recovered in $81 \%$ of cases.

\subsection{Application to GAW 13 Simulated Data}

Aiming to capture the major features of the real Framingham Heart Study data (Levy et al., 2000), the Genetic Analysis Workshop (GAW) 13 (Daw et al., 2003) provided, in Problem 2, 100 replicates of phenotype and genotype data simulated longitudinally on two cohorts with 4,692 individuals clustered in 330 families. It was assumed random ascertainment of families. In this application, we considered only observations at the first time point of the second cohort.

Even though the biological mechanisms underlying these traits is unknown, the data was simulated to reflect reality as much as possible. Therefore, the trait models are complex, far from linear. The true causal model describing relationships among all variables is available in Daw et al. (2003). For ease of reference, Figure 2.4-A shows the true relations among the seven traits considered in this application: height (Ht), weight (Wt), high density lipoprotein (HDL), total cholesterol (Chol), triglycerides (Trig), glucose (Gluc), and systolic blood 
Table 2.2: True and estimated adjacency matrices of the DAGs and of their respective moral graphs in total, genetic, and environmental components, for scenarios 1 to 4. The entries of the estimated adjacency matrices are the percentage of directed edges, followed by the percentage of undirected edges, which is duplicated above and below the main diagonal.

\begin{tabular}{|c|c|c|c|c|c|c|c|c|c|c|c|c|c|c|c|c|c|c|c|}
\hline & & & & & Tota & & & & & & Genet & & & & & & nvironm & nental & \\
\hline & & & rue & & & Estimate & & & rue & & & Estimat & & & rue & & & Estimat & \\
\hline- & & 0 & 0 & $0)$ & $0 / 0$ & $0 / 2$ & $0 / 2\rangle$ & 0 & 0 & $0)$ & $0 / 0$ & $0 / 1$ & $0 / 1)$ & 0 & 0 & $0)$ & $0 / 0$ & $0 / 1$ & $0 / 1)$ \\
\hline 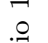 & DAG & 0 & 0 & 0 & $0 / 2$ & $0 / 0$ & $0 / 2$ & 0 & 0 & 0 & $0 / 1$ & $0 / 0$ & $0 / 1$ & 0 & 0 & 0 & $0 / 1$ & $0 / 0$ & $0 / 1$ \\
\hline ฮี & & 1 & 1 & $0)$ & $98 / 2$ & $98 / 2$ & $0 / 0)$ & 1 & 1 & $0)$ & $99 / 1$ & $99 / 1$ & $0 / 0)$ & $(1$ & 1 & $0)$ & $99 / 1$ & $99 / 1$ & $0 / 0$ \\
\hline ల్లె & & 0 & 1 & 1) & $(0$ & 100 & $100)$ & 0 & 1 & $1)$ & $(0$ & 100 & $100)$ & 0 & 1 & 1) & $(0$ & 100 & $100)$ \\
\hline & Moral & 1 & 0 & 1 & 100 & 0 & 100 & 1 & 0 & 1 & 100 & 0 & 100 & 1 & 0 & 1 & 100 & 0 & 100 \\
\hline & & 1 & 1 & $0)$ & 100 & 100 & $\begin{array}{c}0 \\
\end{array}$ & 1 & 1 & $0)$ & 100 & 100 & $0)$ & 1 & 1 & $0)$ & 100 & 100 & 0 \\
\hline$N$ & & 0 & 0 & $0 \bigvee$ & $0 / 0$ & $0 / 100$ & $0 / 100$ & 0 & 0 & $0 \backslash$ & $0 / 0$ & $0 / 100$ & $0 / 100$ & $/ 0$ & 0 & $0\rangle$ & $0 / 0$ & $0 / 100$ & $0 / 100)$ \\
\hline$\therefore$ & DAG & 1 & 0 & 0 & $0 / 100$ & $0 / 0$ & $0 / 0$ & 1 & 0 & 0 & $0 / 100$ & $0 / 0$ & $0 / 0$ & 1 & 0 & 0 & $0 / 100$ & $0 / 0$ & $0 / 3$ \\
\hline త్ర్త్ & & 1 & 0 & 0 人 & $0 / 100$ & $0 / 0$ & $0 / 0$ & 1 & 0 & $0)$ & $0 / 100$ & $0 / 0$ & $0 / 0$ & 1 & 0 & 0) & $0 / 100$ & $0 / 3$ & $0 / 0$ \\
\hline ల్ & & 0 & 1 & 1) & ( 0 & 100 & $100)$ & 0 & 1 & $1)$ & $(0$ & 100 & $100)$ & 0 & 1 & $1)$ & $(0$ & 100 & $100)$ \\
\hline & Moral & 1 & 0 & 0 & 100 & 0 & 0 & 1 & 0 & 0 & 100 & 0 & 0 & 1 & 0 & 0 & 100 & 0 & 3 \\
\hline & Graph & 1 & 0 & $0)$ & 100 & 0 & $0)$ & 1 & 0 & $0)$ & 100 & 0 & $0)$ & 1 & 0 & $0)$ & 100 & 3 & 0 \\
\hline$m$ & & 0 & 0 & $0 \searrow$ & $0 / 0$ & $0 / 1$ & $0 / 100$ & 0 & 0 & $0 \searrow$ & $0 / 0$ & $0 / 60$ & $0 / 100$ & 0 & 0 & $0 \searrow$ & $0 / 0$ & $0 / 88$ & $0 / 100)$ \\
\hline$\therefore$ & DAG & 1 & 0 & 0 & $0 / 1$ & $0 / 0$ & $0 / 100$ & 1 & 0 & 0 & $0 / 60$ & $0 / 0$ & $0 / 100$ & 1 & 0 & 0 & $0 / 88$ & $0 / 0$ & $0 / 100$ \\
\hline 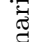 & & 1 & 1 & 0 人 & $0 / 100$ & $0 / 100$ & $0 / 0$ & 1 & 1 & 0 人 & $0 / 100$ & $0 / 100$ & $0 / 0$ & 1 & 1 & $0)$ & $0 / 100$ & $0 / 100$ & $0 / 0$ \\
\hline ల్లే & & 0 & 1 & 1) & $(0$ & 1 & 100 & 0 & 1 & 1) & $(0$ & 60 & $100)$ & 0 & 1 & 1) & $(0$ & 88 & $100)$ \\
\hline & Moral & 1 & 0 & 1 & 1 & 0 & 100 & 1 & 0 & 1 & 60 & 0 & 100 & 1 & 0 & 1 & 88 & 0 & 100 \\
\hline & Graph & 1 & 1 & $0)$ & 100 & 100 & 0 & 1 & 1 & $0)$ & 100 & 100 & $0)$ & 1 & 1 & $0)$ & 100 & 100 & 0 \\
\hline & & 0 & 0 & $0)$ & $0 / 0$ & $0 / 0$ & $0 / 0)$ & 0 & 0 & $0)$ & (0/0 & $0 / 0$ & $0 / 0$ & 0 & 0 & $0)$ & $(0 / 0$ & $0 / 0$ & $0 / 1)$ \\
\hline. & DAG & 0 & 0 & 0 & $0 / 0$ & $0 / 0$ & $0 / 0$ & 0 & 0 & 0 & $0 / 0$ & $0 / 0$ & $0 / 100$ & 0 & 0 & 0 & $0 / 0$ & $0 / 0$ & $0 / 19$ \\
\hline 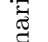 & & 1 & 1 & $0)$ & $100 / 0$ & $100 / 0$ & $0 / 0$ & 0 & 1 & 0 & $0 / 0$ & $0 / 100$ & $0 / 0$ & 1 & 1 & 0 & $81 / 1$ & $81 / 19$ & $0 / 0$ \\
\hline త్ర & & 0 & 1 & 1) & $(0$ & 100 & $100)$ & 0 & 0 & $0)$ & ( 0 & 1 & $1)$ & 0 & 1 & $1)$ & 0 & 99 & 89 \\
\hline & Mor & 1 & 0 & 1 & 100 & 0 & 100 & 0 & 0 & 1 & 1 & 0 & 100 & 1 & 0 & 1 & 99 & 0 & 100 \\
\hline & Graph & 1 & 1 & $0)$ & 100 & 100 & 0 & 0 & 1 & 0 & 1 & 100 & 0 & 1 & 1 & 0 & 89 & 100 & $\begin{array}{c}0 \\
0\end{array}$ \\
\hline
\end{tabular}

pressure (SBP). The variable weight was simulated with a family random effect contributing with $30 \%$ of sex-specific baseline variance. Weight is strongly dependent on height via the body mass index formula. Glucose depends on weight. Triglycerides linearly depends on weight and on the natural logarithm of HDL and of glucose. Cholesterol depends on the base-10 logarithm of triglycerides and of weight. Untreated systolic blood pressures were generated as a function of the natural logarithm of glucose and of a small proportion of weight and cholesterol. Hypertension treatment depends mostly on the age. Smoking and alcohol consumption were generated as environmental variables (i.e., none of the simulated genes had a direct effect on either variable) and as covariates for the other traits. Their values depend on height, sex, and age. This dataset has no missing data.

We applied the proposed method for learning the structure of the directed PGM and its decomposition into genetic and environmental PGMs, over the seven quantitative traits. All these traits were adjusted for additive effects of the following covariates: sex, age, hypertension treatment, cigarettes smoked per day, and quantity of alcohol consumed per week. We did not use the genotype information.

To minimize confounding in the genetic and environmental residuals, it was performed a dimensionality reduction so that the minimum dimension is 10 and the maximum fraction of confounding is $5 \%$.

Since multiple hypothesis tests are performed in the structure learning process, the significance level should be adjusted to control the Type I error. However, to counterbalance 
Table 2.3: True and estimated partial correlation coefficients in the total, genetic, and environmental components, for scenarios 1 to 4. The estimates are represented by their mean \pm standard deviation, followed by the percentage of estimates considered significantly different from 0 at a significance level of 0.005 .

\begin{tabular}{|c|c|c|c|c|c|c|}
\hline & \multicolumn{2}{|r|}{ Total } & \multicolumn{2}{|r|}{ Genetic } & \multicolumn{2}{|r|}{ Environmental } \\
\hline & True & Estimate & True & Estimate & True & Estimate \\
\hline \multirow{6}{*}{ 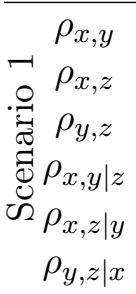 } & 0.000 & $0.006 \pm 0.04(2 \%)$ & 0.000 & $0.011 \pm 0.05(1 \%)$ & 0.000 & $-0.003 \pm 0.07(1 \%)$ \\
\hline & 0.588 & $0.584 \pm 0.02(100 \%)$ & 0.697 & $0.651 \pm 0.04(100 \%)$ & 0.415 & $0.476 \pm 0.06(100 \%)$ \\
\hline & 0.606 & $0.613 \pm 0.02(100 \%)$ & 0.518 & $0.568 \pm 0.05(100 \%)$ & 0.700 & $0.675 \pm 0.04(100 \%)$ \\
\hline & -0.554 & $-0.551 \pm 0.02(100 \%)$ & -0.611 & $-0.578 \pm 0.05(100 \%)$ & -0.495 & $-0.491 \pm 0.05(100 \%)$ \\
\hline & 0.739 & $0.735 \pm 0.02(100 \%)$ & 0.814 & $0.783 \pm 0.03(100 \%)$ & 0.582 & $0.637 \pm 0.05(100 \%)$ \\
\hline & 0.749 & $0.751 \pm 0.01(100 \%)$ & 0.722 & $0.739 \pm 0.03(100 \%)$ & 0.770 & $0.765 \pm 0.03(100 \%)$ \\
\hline \multirow{6}{*}{ 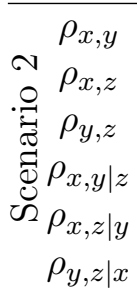 } & -0.634 & $-0.640 \pm 0.02(100 \%)$ & -0.722 & $-0.697 \pm 0.04(100 \%)$ & -0.588 & $-0.611 \pm 0.04(100 \%)$ \\
\hline & 0.683 & $0.689 \pm 0.02(100 \%)$ & 0.603 & $0.649 \pm 0.04$ & 0.763 & $0.741 \pm 0.03(100 \%)$ \\
\hline & -0.433 & $-0.441 \pm 0.02(100 \%)$ & -0.436 & $-0.454 \pm 0$ & -0.449 & $-0.451 \pm 0.06(3 \%)$ \\
\hline & -0.514 & $-0.516 \pm 0.02(100 \%)$ & -0.648 & $-0.597 \pm 0.04(100 \%)$ & -0.435 & $-0.461 \pm 0.05(100 \%)$ \\
\hline & 0.586 & $0.591 \pm 0.02(100 \%)$ & 0.474 & $0.527 \pm 0.05(100 \%)$ & 0.693 & $0.659 \pm 0.04(100 \%)$ \\
\hline & 0.000 & $0.000 \pm 0.03(0 \%)$ & 0.000 & $-0.003 \pm 0.08(0 \%)$ & 0.000 & $0.005 \pm 0.09(3 \%)$ \\
\hline \multirow{5}{*}{ 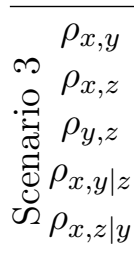 } & -0.613 & $-0.621 \pm 0.03(100 \%)$ & -0.542 & $-0.574 \pm 0.05(100 \%)$ & -0.735 & $-0.700 \pm 0.04(100 \%)$ \\
\hline & -0.759 & $-0.760 \pm 0.01(100 \%)$ & -0.782 & $-0.771 \pm 0.03(100 \%)$ & -0.736 & $-0.747 \pm($ \\
\hline & 0.809 & $0.809 \pm 0.02(100 \%)$ & 0.877 & $0.847 \pm 0.03(100 \%)$ & 0.746 & $0.766 \pm 0.04(100 \%)$ \\
\hline & 0.005 & $-0.015 \pm 0.04(1 \%)$ & 0.424 & $0.314 \pm 0.14(60 \%)$ & -0.408 & $-0.323 \pm 0.11(88 \%)$ \\
\hline & -0.567 & $-0.560 \pm 0.02(100 \%)$ & -0.766 & $-0.663 \pm 0.04(100 \%)$ & -0.435 & $-0.469 \pm 0.06(100 \%)$ \\
\hline$\rho_{y, z \mid x}$ & 0.669 & $0.662 \pm 0.02(100 \%)$ & 0.866 & $0.781 \pm 0.03(100 \%)$ & 0.447 & $0.510 \pm 0.06(100 \%)$ \\
\hline \multirow{6}{*}{ 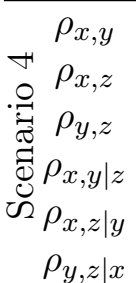 } & 0.000 & $-0.007 \pm 0$ & 0.00 & $-0.006 \pm$ & 0.000 & 0.001 \\
\hline & 0.286 & $0.287 \pm 0.03(100 \%)$ & 0.000 & $0.043 \pm 0.13(0 \%)$ & 0.497 & $14(85 \%)$ \\
\hline & 0.629 & $0.628 \pm 0.02(100 \%)$ & 0.643 & $0.641 \pm 0.04(100 \%)$ & 0.599 & $0.605 \pm 0.05(100 \%)$ \\
\hline & -0.241 & $-0.252 \pm 0.03(100 \%)$ & -0.037 & $-0.041 \pm 0.19(1 \%)$ & -0.426 & $-0.400 \pm 0.08(99 \%)$ \\
\hline & & $0.375 \pm 0.03(100 \%)$ & 0.000 & $0.054 \pm 0.18(1 \%)$ & 0.620 & $0.495 \pm 0.17(89 \%)$ \\
\hline & 0.656 & $0.658 \pm 0.02(100 \%)$ & 0.643 & $0.653 \pm 0.04(100 \%)$ & 0.690 & $0.686 \pm 0.05(100 \%)$ \\
\hline
\end{tabular}


the loss of statistical power due to the confounding correction, we considered a significance level of $5 \%$. Even though, as we will describe in the following, the false positive rate was around $5 \%$.

Figures 2.4B-D show the results for the true edges in, respectively, total, genetic, and environmental graphs, considering 100 replicates. Adjacent to the edges are shown the partial correlation coefficients (mean \pm standard deviation), obtained by conditioning on the necessary variables to block all indirect paths. In addition, enclosed by parentheses is the percentage of cases in which the edge was learned as illustrated in the graph, followed by the percentage of cases in which the edge was detected, but was left unoriented or was oriented in the other direction. The edge thickness represents the percentage in which the edge was detected: the thicker is the edge, the higher is the percentage of cases in which the edge was identified. For unshielded colliders, it is also shown information on the partial correlation given the collider. We decided to omit the number of false positive detections since it is very small (less than 5\%) for every possible edge in the three graphs.

Particularly, we can note a large number of false negative detections in the total graph (Figure 2.4-B). The estimated magnitude of the respective partial correlation coefficients are too low (close to zero). Considering that the proposed methods were developed under the linearity assumption and that it is hard to detect linear trends in logarithmic functions, particularly with large bases, such as natural logarithm and that in base 10, true connections are likely to be considered weak. In the case of linear relationships, the algorithm was able to identify the true edges in $100 \%$ of cases.

The orientation of the edges depends on the presence of unshielded colliders. In the true causal PGM, there are three unshielded colliders: HDL $\rightarrow$ Trig $\leftarrow$ Wt, HDL $\rightarrow$ Trig $\leftarrow$ Gluc, and Gluc $\rightarrow$ SBP $\leftarrow$ Chol. For the total component, the edge HDL $\rightarrow$ Trig can be oriented using the marginal and partial correlations of the triplets (HDL, Trig, Wt) and (HDL, Trig, Gluc). Due to this redundancy, it could be recovered with a high accuracy (99\% of cases). The edge Gluc $\rightarrow$ Trig is oriented using the information only on the triplet (HDL, Trig, Gluc). Since $\hat{\rho}_{\text {Gluc,HDL Trig }}$ is weak $(0.091 \pm 0.03$, being significantly different from zero only in $69 \%$ of cases), the edge was correctly oriented only in $73 \%$ of cases. The IC/PC algorithm is also hindered by ambiguous triplets. When making $\mathrm{Wt} \rightarrow$ Trig, since the connection between Wt and Gluc is very weak and could not be detected in $97 \%$ of cases, the formation Wt $\rightarrow$ Trig $\leftarrow$ Gluc was determined as an unshielded collider. However, $\hat{\rho}_{G l u c, W t \mid \text { Trig }}$ is too weak to be different from zero, leading to a contradiction. In these cases, the edge is left unoriented. Even faced with these ambiguities, the algorithm identified correctly the edge $\mathrm{Wt} \rightarrow$ Trig in $94 \%$ of cases. The edge between $\mathrm{Wt}$ and $\mathrm{Ht}$ could not be oriented using information from any of the previous oriented edges.

Although the contribution of genetic and environmental factors on the connections was not provided by GAW 13, we also applied the proposed methods to learn the decomposition of the total PGM into genetic and environmental PGMs, shown, respectively, in Figures 2.4$\mathrm{C}$ and D. Since the variable weight was simulated with a family random effect, its edges were 
identified mostly in the genetic PGM. The edge between Wt and Trig was detected in 100\% of cases in the genetic component, but only in $3 \%$ of cases in the environmental component. Also, the edge between $\mathrm{Wt}$ and $\mathrm{Ht}$ was detected in $100 \%$ of cases in the genetic component and in $25 \%$ of cases in the environmental component. Although with a small detection rate due to the weak effect, the edge between Wt and SBP was detected in $19 \%$ of cases in the genetic component and never was detected in the environmental component. That suggests that these associations have high heritability and are due to pleiotropic effects of genes on the variables in question. The edges between Trig and HDL and between Trig and Gluc were identified in both genetic and environmental components, suggesting that these relationships have a heritable genetic component, but are also affected by environmental factors.

(A) True Graph

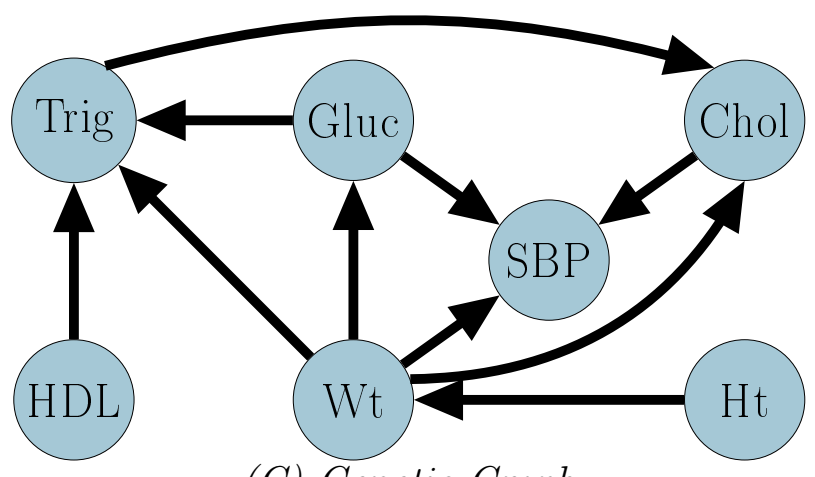

(C) Genetic Graph

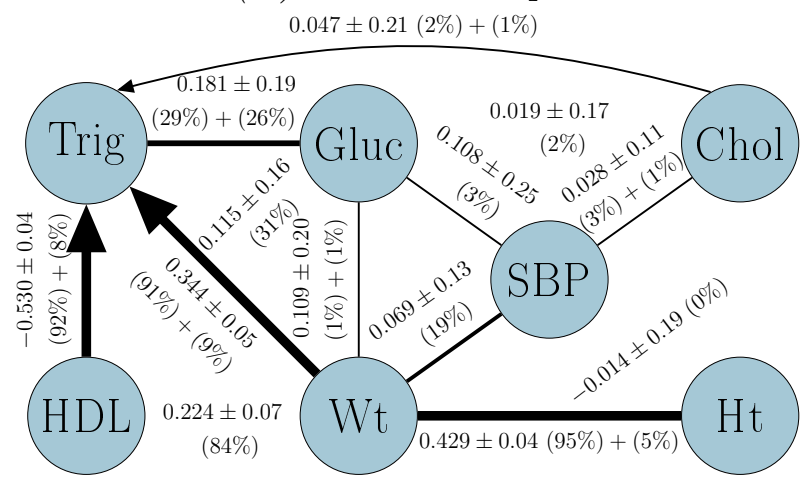

(B) Total Graph

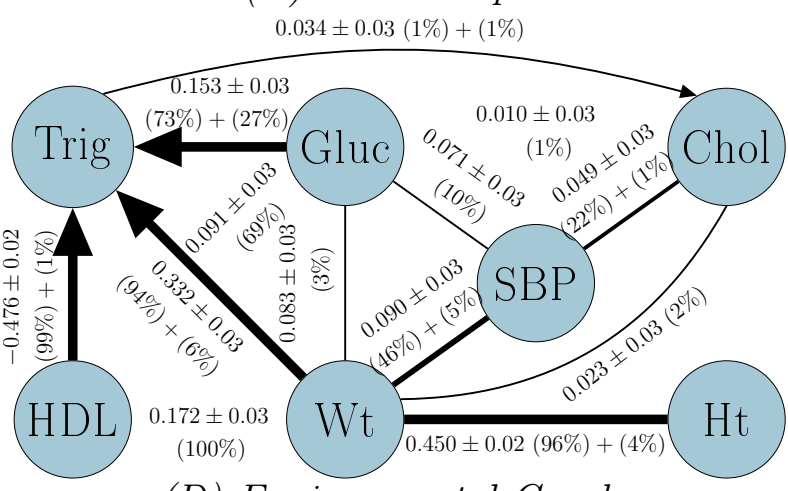

(D) Environmental Graph

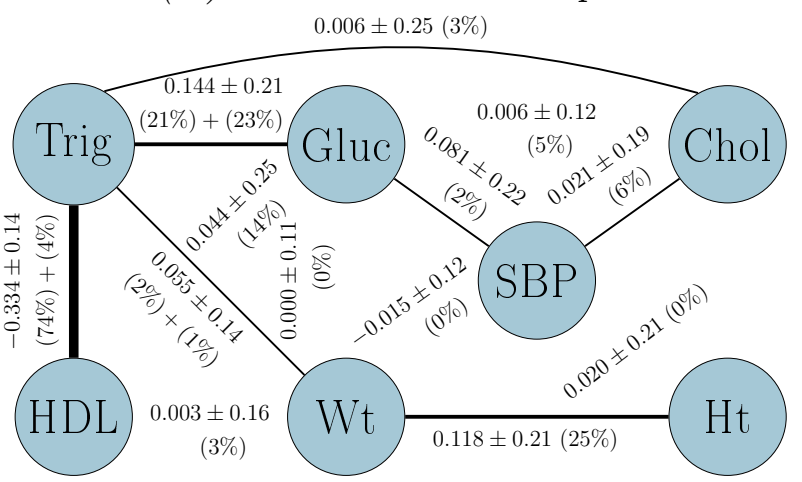

Figure 2.4: (A) is true causal graph describing the relationships among the traits in GAW 13 dataset. Simulation results, considering 100 replicates, for these true edges are shown for the learned (B) total, (C) genetic, and (D) environmental directed PGMs. Adjacent to the edges are the mean and standard deviation of the direct effect (obtained by conditioning on the necessary variables to block all indirect paths), as well as, enclosed by parentheses, the percentage of cases in which the edge was learned as illustrated in the graph, followed by the percentage of cases in which the edge was left unoriented or was oriented in the other direction. The edge thickness represents the percentage in which the edge was detected. A significance level of 5\% was used. For unshielded colliders, it is also shown information on the partial correlation given the collider. 


\subsection{Application to Baependi Heart Study Dataset}

The number of medical problems related to the metabolic syndrome has been increasing rapidly worldwide. Currently, the metabolic syndrome is considered as the most prominent risk factor for cardiovascular diseases and it has become a major public health problem in Brazil. It is a multifactorial disease characterized by at least three of the five following metabolic disturbances: abdominal obesity (increased waist circumference), elevated blood pressure, elevated fasting blood glucose, high triglycerides, and low high-density lipoprotein cholesterol (Grundy et al., 2005).

The Baependi Heart Study is a family-based, highly admixed cohort study carried out in a rural Brazilian town (de Oliveira et al., 2008). It was designed to investigate cardiovascular diseases and their associated factors. To understand the mechanism behind the metabolic syndrome phenotypes, we applied the proposed methods to data from 1,666 individuals in 81 families and recovered the structure of the undirected and directed PGM for the following five phenotypes: glucose (Gluc), triglycerides (Trig), high-density lipoprotein cholesterol $(H D L)$, waist circumference $(W C)$, and systolic blood pressure $(S B P)$. In addition, we present a decomposition of the total PGMs into genetic (between-family) and environmental (within-family) PGMs.

In the regression analyses, we considered the main effects for sex, age, and squared age, as well as, the interaction effect between sex and age. To correct for population stratification, we also considered as covariates two measurements of the Baependi population ancestry derived from the two first principal components of heritability for 8,764 single nucleotide polymorphisms (SNPs) (De Andrade et al., 2015; Oualkacha et al., 2012).

Considering that may be possible to have a significant loss of statistical power in the confounding correction when the genetic and environmental effects have opposite signs or when one of them is zero, we decided, for this application, show the results using a significance level of $5 \%$. We noticed that using a smaller significance level, some edges are no longer detected, making several triples ambiguous in the learning process of the $\mathrm{PC} / \mathrm{IC}$ algorithm. Thus, false positive results can be obtained and should be carefully analyzed.

The undirected PGMs was recovered by assigning an edge between every pair of variables $(X, Y)$ such that the partial correlation coefficient between $X$ and $Y$ given the other variables, $\boldsymbol{V} \backslash\{X, Y\}$, is significant. In Table 2.4, we show the estimates and p-values for the edges identified in at least one of the three learned PGMs (total, genetic, or environmental). The results for the significant edges are shown in bold. Figure 2.5 illustrates the learned (A) total, (B) genetic, and (C) environmental undirected PGMs. The partial correlation coefficients are shown adjacent to the edges. Significant associations are represented by full edges. If two variables are not significantly associated in the PGM in question, but are associated in one of the other two learned PGMs, this connection is represented by a dashed edge.

In the three PGMs, it was identified that $W C$ is negatively correlated with $H D L$ and positively correlated with $S B P$ and with Trig. The positive associations between Gluc 
A)

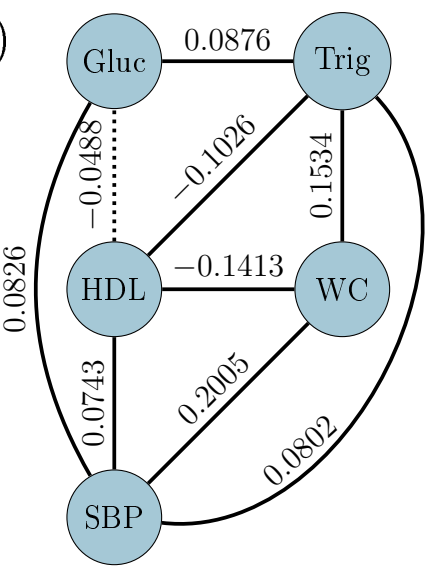

B)

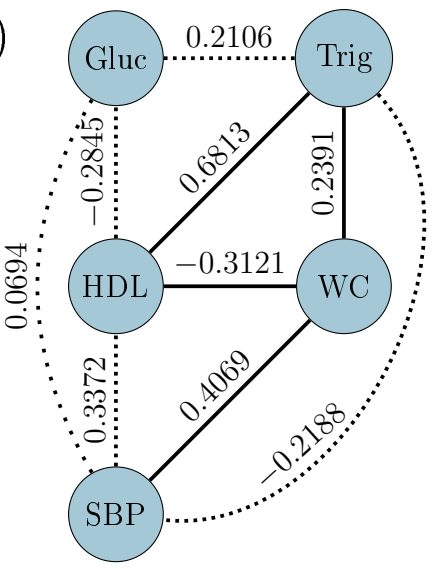

C)

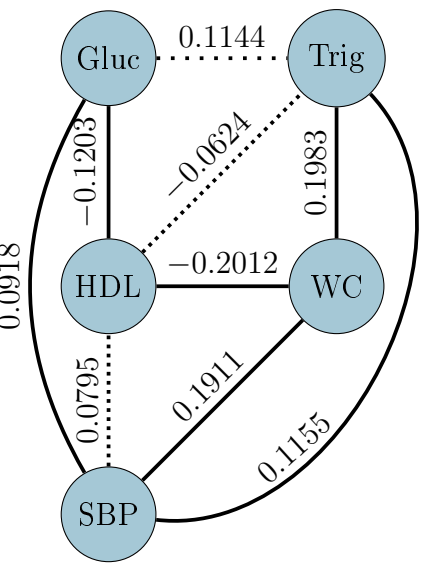

Figure 2.5: Total (A), genetic (B), and environmental $(C)$ undirected PGMs estimated by applying the proposed method to the metabolic syndrome phenotypes from Baependi Heart Study. Five phenotypes were considered: glucose (Gluc), triglycerides (Trig), high-density lipoprotein cholesterol (HDL), waist circumference (WC), and systolic blood pressure (SBP).

and Trig and between $H D L$ and $S B P$ are significant only in the total PGM. Also, SBP is positively correlated with Gluc and Trig in total and environmental PGMs, but those associations are not significant in the genetic PGM. The association between Trig and $H D L$ is negative in total PGM, positive in genetic PGM, and non-significant in the environmental PGM. Finally, the negative association between Gluc and $H D L$ was considered significant only in the environmental PGM.

The CPDAGs for the total, genetic, and environmental PGMs were learned as shown, respectively, in Figure 2.6-A, B and C. Estimates for partial correlation coefficients representing the direct effects for the learned PGMs are shown in bold type in Table 2.5.

A)

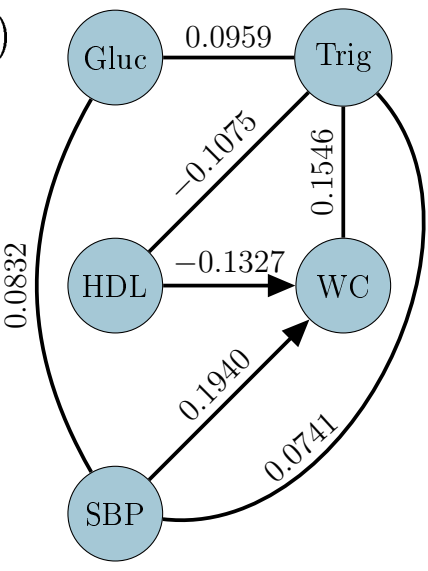

B)

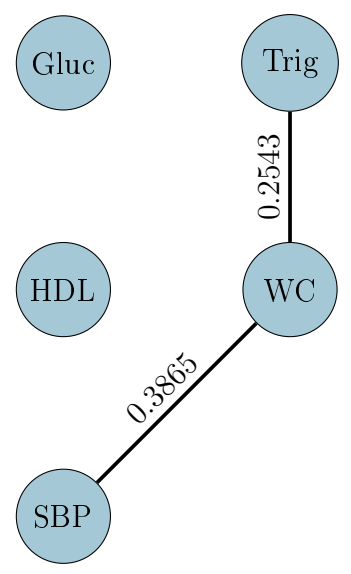

C)

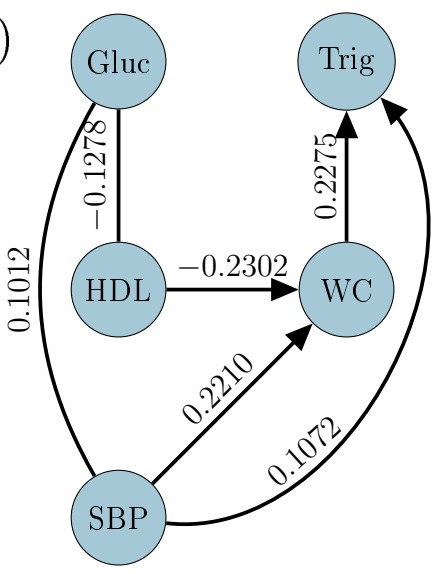

Figure 2.6: Total $(A)$, genetic (B), and environmental $(C)$ directed PGMs estimated by applying the proposed method to the metabolic syndrome phenotypes from Baependi Heart Study. Five phenotypes were considered: glucose (Gluc), triglycerides (Trig), high-density lipoprotein cholesterol (HDL), waist circumference (WC), and systolic blood pressure (SBP).

In the total and environmental CPDAGs, it was identified the unshielded collider $H D L \rightarrow$ $W C \leftarrow S B P\left(\rho_{t_{H D L, S B P}}=0.0254, \mathrm{p}\right.$-value $=0.4001, \rho_{t_{H D L, S B P \mid\{W C\}}}=0.0617, \mathrm{p}$-value $=$ $0.0408 ; \rho_{e_{H D L, S B P}}=-0.0029, \mathrm{p}$-value $=0.9611, \rho_{e_{H D L, S B P \mid\{W C\}}}=0.3559, \mathrm{p}$-value $\left.=0.0152\right)$ 
That suggests that the edge between HDL and SBP, that was significant only in the total undirected PGM, is a spurious association due to the conditioning on the collider $W C$. In the environmental CPDAG, it was possible to determine the direction of the edge $W C \rightarrow$ Trig (the only possibility considering that the triplet is not an unshielded collider) and of the edge $S B P \rightarrow$ Trig (the other direction would generate a cycle, contradicting the assumption of the PGM being acyclic).

Only considering the genetic PGM, it is not possible to determine the direction of any edge in the genetic PGM. The following three formations have the same joint distribution probability and, thus, are not statistically distinguishable: $S B P \rightarrow W C \rightarrow$ Trig, $S B P \leftarrow$ $W C \leftarrow$ Trig, and Trig $\leftarrow W C \rightarrow S B P$. However, by looking at the environmental PGM, one may conjecture that the true formation is $S B P \rightarrow W C \rightarrow$ Trig.

The edges connecting HDL to WC and to Trig in the genetic undirected PGM (Figure 2.5-B) are no longer present in the genetic directed acyclic PGM (Figure 2.6-B). As shown in Table 2.5, Trig and $H D L$ are significantly correlated in the genetic PGM if conditioned on a set containing $W C$ and $S B P$. Also, $W C$ and $H D L$ are significantly correlated in the genetic PGM if conditioning on a set containing $S B P$ or Trig. Marginally, for example, these two pair of variables are uncorrelated.

In the total PGM, the association between Gluc and $H D L$ is explained by the indirect effect through the mediator Trig $\left(\rho_{\text {Gluc }, H D L}=-0.0598\right.$, p-value $=0.0474 ; \rho_{\text {Gluc }, H D L \mid\{\text { Trig }\}}=$ $-0.0457, \mathrm{p}$-value $=0.1298$ ). Thus, only considering the total PGM, it is not possible to determine the directions of this triplet. By supposing that the direction of the edge between $W C$ and Trig in the total CPDAG is the same as in the environmental CPDAG, i.e., $W C \rightarrow$ Trig, we may conjecture that $H D L \rightarrow$ Trig (otherwise would generate a cycle) and, consequently, Trig $\rightarrow$ Gluc (the triplet (WC, Trig, Gluc) is not an unshielded collider). However, by supposing that $S B P \rightarrow$ Trig (same direction identified in the environmental CPDAG), we would have that $S B P \rightarrow G l u c$ (otherwise would generate a cycle), but we would also have an ambiguity because the formation $H D L \rightarrow$ Trig $\leftarrow S B P$ does not have the conditional statements of an unshielded collider. We have that $H D L$ and $S B P$ are marginally uncorrelated $\left(\rho_{t_{H D L, S B P}}=0.0254\right.$, p-value $\left.=0.4001\right)$, but they do not became correlated when conditioned on Trig $\left(\rho_{t_{H D L, S B P \mid\{T r i g\}}}=0.0415\right.$, p-value $\left.=0.1691\right)$. We can note, however, that the partial correlation between $H D L$ and $S B P$ increases when conditioning on Trig, compared when conditioning only on $W C\left(\rho_{t_{H D L, S B P \mid\{W C\}}}=0.0617\right.$, p-value $=0.0408 ; \rho_{t_{H D L, S B P\{W C, T r i g\}}}=0.0708, \mathrm{p}$-value $\left.=0.0188\right)$. That suggests that Trig may still be a collider.

In the estimated CPDAG for the environmental PGM, the triplet (SBP, Gluc, HDL) is not an unshielded collider. Thus, the possible formations for this triplet are: $S B P \leftarrow$ Gluc $\leftarrow H D L, S B P \rightarrow G l u c \rightarrow H D L$, and $S B P \leftarrow$ Gluc $\rightarrow H D L$. By supposing that $S B P \rightarrow$ Gluc (a conjecture just made for the total PGM), the only possible formation would be $S B P \rightarrow$ Gluc $\rightarrow H D L$. 
Table 2.4: Partial correlation estimation results for learning total, genetic, and environmental undirected PGMs among the five metabolic syndrome phenotypes from Baependin Heart Study: glucose (Gluc), triglycerides (Trig), high-density lipoprotein cholesterol (HDL), waist circumference $(W C)$, and systolic blood pressure $(S B P)$. For every pair of phenotypes, $(X, Y)$, conditioning on the others, $\boldsymbol{V} \backslash\{X, Y\}$, columns show, respectively, partial correlation coefficients and p-values in the total PGM, and partial correlation coefficients, p-values, and sample sizes after dimension reduction in confounding correction, in genetic and environmental PGMs. The significant edge coefficients (direct effects) are shown in bold.

\begin{tabular}{|c|c|c|c|c|c|c|c|c|c|}
\hline \multicolumn{2}{|c|}{ Vertices } & \multicolumn{2}{|c|}{$\rho_{t_{X, Y \mid \boldsymbol{V} \backslash\{X, Y\}}}$} & \multicolumn{3}{|c|}{$\rho_{g_{X, Y \mid \boldsymbol{V} \backslash\{X, Y\}}}$} & \multicolumn{3}{|c|}{$\rho_{e_{X, Y \mid \boldsymbol{V} \backslash\{X, Y\}}}$} \\
\hline$X$ & $Y$ & Estimate & P-value & Estimate & P-value & $\mathrm{N}$ & Estimate & P-value & $\mathrm{N}$ \\
\hline WC & HDL & -0.1413 & 0.0000 & -0.3121 & 0.0135 & 62 & -0.2012 & 0.0000 & 605 \\
\hline WC & Trig & 0.1534 & 0.0000 & 0.2391 & 0.0388 & 75 & 0.1983 & 0.0000 & 550 \\
\hline WC & SBP & 0.2005 & 0.0000 & 0.4069 & 0.0010 & 62 & 0.1911 & 0.0000 & 733 \\
\hline Gluc & HDL & -0.0488 & 0.1054 & -0.2845 & 0.0976 & 35 & -0.1203 & 0.0030 & 605 \\
\hline Gluc & Trig & 0.0876 & 0.0037 & 0.2106 & 0.0847 & 68 & 0.1144 & 0.2593 & 99 \\
\hline Gluc & SBP & 0.0826 & 0.0061 & 0.0694 & 0.6469 & 46 & 0.0918 & 0.0240 & 605 \\
\hline HDL & Trig & -0.1026 & 0.0007 & 0.6813 & 0.0147 & 12 & -0.0624 & 0.1255 & 605 \\
\hline HDL & SBP & 0.0743 & 0.0137 & 0.3372 & 0.1460 & 20 & 0.0795 & 0.0508 & 605 \\
\hline Trig & SBP & 0.0802 & 0.0078 & -0.2188 & 0.1440 & 46 & 0.1155 & 0.0097 & 500 \\
\hline
\end{tabular}


Table 2.5: Partial correlation estimation results for learning total, genetic, and environmental directed acyclic PGMs among the five metabolic syndrome phenotypes from Baependi Heart Study: glucose (Gluc), triglycerides (Trig), high-density lipoprotein cholesterol (HDL), waist circumference $(W C)$, and systolic blood pressure $(S B P)$. For every pair of phenotypes, $(X, Y)$, conditioning on the set $\boldsymbol{S}$ of variables necessary to block all indirect paths, columns show, respectively, partial correlation coefficients and p-values in the total PGM, and partial correlation coefficients, p-values, and sample sizes after dimension reduction in confounding correction, in genetic and environmental PGMs. The edge coefficients (direct effects) are shown in bold.

\begin{tabular}{|c|c|c|c|c|c|c|c|c|c|c|}
\hline \multicolumn{3}{|c|}{ Vertices } & \multicolumn{2}{|c|}{$\rho_{t_{X, Y \mid S}}$} & \multicolumn{3}{|c|}{$\rho_{g_{X Y Y Y}}$} & \multicolumn{3}{|c|}{$\rho_{e_{X, Y \mid S}}$} \\
\hline$X$ & $Y$ & $S$ & Estimate & P-value & Estimate & P-value & $\mathrm{N}$ & Estimate & $\mathrm{P}$-value & $\mathrm{N}$ \\
\hline $\mathrm{WC}$ & HDL & & -0.1565 & 0.0000 & -0.2397 & 0.0606 & 62 & -0.2247 & 0.0000 & 605 \\
\hline WC & HDL & Trig & -0.1327 & 0.0000 & -0.4202 & 0.0409 & 24 & & 00 & 550 \\
\hline $\mathrm{WC}$ & HDL & SBP & -0.1642 & & & 0.0252 & 22 & 302 & 0.0000 & 605 \\
\hline $\mathrm{WC}$ & Trig & & 0.1922 & 0.0000 & 0.2543 & 0.0277 & 75 & 450 & 0.0000 & 605 \\
\hline WC & Trig & SBP & 0.1720 & & & & 56 & 75 & 00 & 605 \\
\hline WC & Trig & HDL,SBP & 0.1546 & 0.0000 & 0.29 & 0.0255 & 56 & & 0.0000 & 550 \\
\hline WC & SBP & & & & & & 62 & & & 806 \\
\hline WC & SBP & Trig & 0.1940 & 0.0 & & & 62 & & & 806 \\
\hline WC & SBP & Gluc & & & & & 62 & & & 806 \\
\hline Gluc & HDL & & -0.0598 & 0.0 & -0.2 & & 35 & 278 & 0.0016 & 605 \\
\hline Gluc & HDL & Trig & -0.0457 & & & & 75 & & & 455 \\
\hline Gluc & Trig & & 0.1062 & & & & 56 & & & 194 \\
\hline Gluc & Trig & SBP & 0.0959 & 0.00 & & & 75 & & & 376 \\
\hline Gluc & SBP & & 0.0951 & & & & 51 & 12 & 90 & 666 \\
\hline Gluc & SBP & Trig & 0.0832 & & & & 51 & & & 666 \\
\hline HDL & Trig & & -0.1296 & 0.0000 & & 08 & 29 & 091 & 072 & 605 \\
\hline HDL & Trig & WC & -0.1025 & 0.00 & & & 38 & 48 & 177 & 500 \\
\hline HDL & Trig & WC,SBP & -0.1075 & 0.0004 & 0.6284 & 0.0214 & 13 & -0.0622 & 0.1264 & 605 \\
\hline HDL & SBP & & 0.0254 & 0.4001 & & & 10 & 29 & 811 & 283 \\
\hline HDL & SBP & WC & 0.0617 & & & & 1100 & & & 46 \\
\hline HDL & SBP & Trig & 0.0415 & & -0.0003 & & 68 & -0.0007 & 0.9927 & 160 \\
\hline HDL & SBP & WC,Trig & 0.0708 & & & & 22 & & & 68 \\
\hline Trig & SBP & & 0.1193 & 0.0001 & -0.0944 & 0.4656 & 62 & 0.1432 & 0.0008 & 550 \\
\hline Trig & SBP & WC & 0.0824 & & & & 51 & 0.1072 & 0.0119 & 550 \\
\hline Trig & SBP & WC,Gluc & 0.0741 & 0.0139 & -0.1862 & 0.1474 & 62 & 0.1022 & 0.0165 & 550 \\
\hline
\end{tabular}




\subsection{Discussion and Perspectives for Future Work}

Studies of complex diseases are challenging because they are polygenic and multifactorial. The learning of the association or causal network among multiple phenotypes and of its decomposition into genetic and environmental components may help us to understand the underlying mechanisms and the roles of polygenic (multiple genes) and environmental factors. Also, it may help to decide between a genetic and an environmental treatment.

Considering that genetic associations may be due to genes that have a pleiotropic effect on the variables in question, gene-mapping techniques should be applied for finding the genes responsible for such associations and then proposing a genetic interventions for therapeutic purposes. Learning about the causal relationships among the phenotypes may also contribute to gene-mapping studies. In cases involving pleiotropy of the mapped locus, modeling multiple phenotypes simultaneously can dramatically increase power in linkage analysis, compared with analyses using each phenotype separately. In particular, several authors have shown that the greatest power increases occur when the genetic correlation between phenotypes (induced by the locus) is opposite in sign to the environmental correlation (induced by residual factors) (Allison et al., 1998; Amos et al., 2001; Evans, 2002).

In this work, based on univariate polygenic mixed models, we have proposed reliable and unconfounded partial correlation tests for assessing the significance of total-sample, genetic (between-family) and environmental (within-family) partial correlations when using family data. Under the additional assumptions of normal distribution with homoscedastic families and linear relationships among the phenotypes, these tests were used to develop algorithms that learn, from observational Gaussian family data, undirected and directed PGMs among multiple phenotypes and also a decomposition of the learned PGMs into genetic and environmental PGMs.

To learn undirected and directed PGMs, multiple tests are performed to get information about the conditional independence relations among the variables. Due to this multiple testing problem, it is recommended a significance level adjustment to control the proportion of Type I errors. However, a precise adjustment is very complicated to be derived because the hypothesis are not independent and also because the correction for minimizing the confounding in the predicted effects and conditional residuals of the polygenic mixed models may lead to a significant loss of statistical power. Since false negative detections lead to a sequence of wrong decisions in the causal learning process, we notice in our studies that the methods are quite sensible to the significance level adjustment. Also, this problem gets more complicated as the data dimensionality increases. Thus, more studies are necessary to derive criteria for selecting the maximum fraction of counfouding and the significance level to be used so that the best possible results are drawn.

It is important to remark that methods for studying causality from observational data may require assumptions that are hard to verify, such as causal sufficiency (all common causes of the measured variables were measured), linear relationships and absence of cycles 
(feedback loops). Thus, we have to be aware that results may be unreliable if some assumption is violated. However, results drawn from observational studies may still be helpful in generating new hypotheses as well as in designing RCTs to address specific questions.

To learn causal PGMs, we used the proposed partial correlation tests as d-separation oracles in the IC/PC algorithm. Some of its variants, such as the FCI and RFCI algorithms (both implemented in the pcalg $R$ package), relax the assumption of causal sufficiency by indicating potential latent causes for pairs of observed variable. Since these algorithms are also conditional independence learners, we can extend them by using the proposed partial correlation tests as their d-separation oracles and thus learn from family data even when the assumption of causal sufficiency is violated.

As shown in Section 2.3.2, partial correlation coefficients can also be estimated by the inverse covariance matrix. Thus, considering family data, genetic and environmental partial correlation coefficients could be obtained from estimates of the covariance matrices of the multivariate polygenic linear mixed model. Oualkacha et al. (2012) has proposed efficient ANOVA estimators for these covariance matrices. However, as they are based on the method of moments, we notice that the estimates are often biased, being on the boundary or even outside of the parameter space. To assess the significance of the partial correlation coefficients, Fisher's z-test can be used and, to account for the correlation between observations, the degrees of freedom of the covariance estimates can be used as effective sample sizes. However, from preliminary results using that approach (not presented here), we noticed that the p-values were underestimated, particularly for the genetic component. An advantage of our approach to that based on the precision matrices is that we can adjust the variables for covariates and perform covariate selection in the univariate polygenic models, taking into account the familial dependence structure.

Considering that our proposed methods learn multivariate distributions from univariate polygenic linear mixed models, as future works, we intend to leverage the flexibility and scalability offered by them.

By incorporating penalties in the models, such as the $\ell_{1}$-norm used in the LASSO regressions, it is possible to improve computational time of our methods and efficiently learn high dimensional sparse PGMs.

We can also consider a more complex decomposition of the PGMs by including multiple random factors in the mixed model for modeling, for example, temporal and spatial dependence, within-household clustering (due to shared environmental factors, such as lifestyle or household conditions, that influencing individuals from the same household, making them similar regardless of genetic relatedness), and also interactions between all these random factors. This can help to explain better how pleiotropic genes induce the associations in genetic graph. For example, we may determine that these associations are due to sex- or age-specific genes or even induced by gene-environment interactions. Considering the fictitious causal model among Exercise, Stress, and Depression in Figure 2.3, differences between the genetic and environmental components could be explained by a gene-environment interaction. Thus, 
a more discriminative decomposition of the covariance matrix could help to elucidate the nature of each association and to determine potential genes affecting these three variables.

In addition, since it is common that only the equivalence class of the true underlying causal PGM can be inferred from observational data, we can incorporate information on known genetic causal variables in the learning process to help to distinguish between PGMs that would otherwise be Markov equivalent. Also, by exploiting the concept of Mendelian Randomization, genetic variants can be used as instrumental variables, improving estimation of the causal effects. A detailed exposition on the theory behind Mendelian Randomization and the use of genetic variants as instrumental variables for learning causal models can be found at Ribeiro et al. (2016).

Finally, we intend to exploit the generalized mixed models in family-based designs to develop more general partial correlations, allowing us to learn PGMs over variables that are not only normally distributed. 
40 LEARNING GENETIC AND ENVIRONMENTAL GRAPHICAL MODELS FROM FAMILY DATA 


\section{Chapter 3}

\section{Granger Causality Between Graphs}

Graphs have been extensively used to model high-dimensional systems with complex dependence structures, including brain networks. However, many systems are dynamic and a new notion of Granger causality becomes necessary to study the interdependence structure among time series of graphs. By assuming that graphs are generated by models whose parameters are random variables, we define that a time series of graphs $G_{i, t}$ does not Granger cause another time series of graphs $G_{j, t}$ if the models parameters for $G_{i, t}$ does not Granger cause the model parameters for $G_{j, t}$. Usually, the model parameters are unknown, but, for several random graph models, the spectral radius (the largest eigenvalue of the adjacency matrix) is a function of the model parameters. Based on this idea, we propose to identify Granger causality between time series of graphs by fitting a vector autoregressive model to the time series of the ranked spectral radii. We evaluate the accuracy of the method by simulation studies and present two applications: one investigating brain-brain interaction of two musicians while playing a violin duo, and another showing differential connectivity from the right to the left brain hemisphere in autistic subjects.

\subsection{Introduction}

In many fields of study, such as biology, economics, and social science, researchers have to deal with very large spatio-temporal datasets. In many cases, collected data are clustered in meaningful ways and inferences on the relationships among the clusters over time may help researchers to understand how is the dynamics of the entire system. For example, in neuroscience, functional Magnetic Resonance Imaging (fMRI) data consist of blood-oxygenationlevel-dependent (BOLD) measurements for several hundreds of thousands voxels obtained at hundreds of times. However, functional brain networks (FBNs) are typically built by aggregating the voxels into larger regions of interest (pre-defined clusters of anatomically or functionally connected brain regions) and then estimating association measures between the BOLD signals of these regions. Massive datasets with a complex spatial and temporal structure are also found in others research fields, such as economics, where stock prices of hundred 
or thousands companies can be clustered according their economic sectors, or in social science, where people are organized in communities. Thus, considering that the within-cluster associations can be represented by graphs and that the pattern of these associations can change over time, we can understand the structure and dynamics of the whole system by a between-graph analysis, such as, a Granger causality analysis among time series of graphs.

Based on spectral graph theory and under the assumption that graphs are generated by mathematical models whose parameters are random variables, Fujita et al. (2017) present a new notion of dependence among random graphs. The treatment of the parameters as random variables is common in Bayesian data analysis. The idea is that the parameter value of the model used to generate the graph is drawn from the prior distribution (Gelman et al., 2013). Specifically, Fujita et al. (2017) define that two graphs are independent when the random variables representing their parameters are independent. Thus, a simple way to test whether two graphs are independent is to test the probabilistic dependence between the parameters of their models. However, in practice, the true model is rarely known, and consequently the random variables representing the parameters cannot be predicted.

By the spectral graph theory, it is known that the spectral radius (the largest eigenvalue of the adjacency matrix of the graph) is a function of the parameters for several undirected random graph models, such as Erdös-Rényi, geometric, regular, Barabási-Albert, and WattsStrogatz (Alon, 1986; Bordenave, 2008; Dorogovtsev et al., 2003; Füredi and Komlós, 1981; Van Mieghem, 2010). For example, the spectral radius of a Erdös-Rényi random graph, defined by the parameters $n$ and $p$ representing, respectively, the number of vertices and the probability of connecting two vertices, converges to $n p-2 p+1$ (Füredi and Komlós, 1981). Also, the spectral radius of a regular random graph, in which all vertices have the same number (deg) of incident edges, i.e., the same degree, is equal to deg (Alon, 1986). Thus, the spectral radius captures topological features of several random graphs.

By leveraging this relation between spectral radius and the model parameters, Fujita et al. (2017) propose to test correlation between two samples of graphs by testing the Spearman's rank correlation between the samples of the respective spectral radii. The Spearman's rank correlation coefficient is defined as the Pearson correlation coefficient between the ranked variables. The authors suggest this dependence measure mainly because it is robust to outliers and it does not require assumptions of linearity in the relationship between variables (it can identify monotonic nonlinear associations).

Thus, inspired by Fujita et al. (2017), we present in Section 3.2.2 a method that identifies Granger causality among time series of undirected graphs by fitting a VAR model on the time series of the ranked spectral radii. We call it gVAR, which stands for VAR model for graphs.

The gVAR method was evaluated by the simulation study described in Section 3.2.5, with time series of undirected random graphs generated by Erdös-Rényi, geometric, regular, Barabási-Albert, and Watts-Strogatz random models (Barabási and Albert, 1999; Bender, 1974; Erdôs and Rényi, 1959; Gilbert, 1961; Watts and Strogatz, 1998). In the simulated 
scenarios, all graphs were generated by the same random graph model and the model parameters follow a specific VAR process. Results in Section 3.3.1 show that the gVAR method has considerably high power, particularly for large graphs and long time series, and controls for Type I error. Also, we show that, for identifying Granger causality between graphs, using the spectral radius as a single feature of graph is indeed better than using other common measures, such as, the average degree, eigenvector, betweenness, and closeness centralities.

We illustrate the applicability of the gVAR method by studying two real biological problems, described in Sections 3.2.6 and 3.2.7. In the first application, we analyzed real functional Near Infrared Spectroscopy (fNIRS) signals simultaneously acquired from two musicians while playing a violin duo (Balardin et al., 2017) to study their brain-brain interaction. As shown in Section 3.3.2, we identified a Granger causality from the FBN of the older and more experienced violinist to the FBN of the younger and less experienced violinist. In the second application, we analyzed resting state functional Magnetic Resonance Imaging (rsfMRI) from 737 subjects of the Autism Brain Imaging Data Exchange I (ABIDE I) dataset to study differential connectivity between the brain hemispheres in autistic subjects. In Section 3.3.3, we show that the loss of functional connectivity from the right to the left brain hemisphere as age advances is significantly higher in subjects with autism.

\subsection{Materials and Methods}

\subsubsection{Preliminary Concepts}

\subsubsection{Graph and Spectral Radius of the Adjacency Matrix}

A graph, $G=(\mathbf{V}, \mathbf{E})$, is a pair composed of a vertex set $\mathbf{V}=\left\{V_{1}, \ldots, V_{n}\right\}$ and an edge set $\mathbf{E} \subseteq\left\{\left(V_{i}, V_{j}\right): V_{i}, V_{j} \in \mathbf{V}\right\}$. In an undirected graph, each edge $\left(V_{i}, V_{j}\right)$ represents a undirected connection between the vertices $V_{i}$ and $V_{j}$.

Any undirected graph $G$ with $n$ vertices can be represented by its adjacency matrix $\mathbf{A}^{G}$, a symmetric $n \times n$ matrix with elements $A_{i j}^{G}$ equal to 1 if the edge $\left(V_{i}, V_{j}\right) \in \mathbf{E}$ and 0 otherwise. The spectrum of $G$ is the set of eigenvalues of its adjacency matrix $\mathbf{A}^{G}$. Since real symmetric matrices only have real eigenvalues, an undirected graph with $n$ vertices has $n$ real eigenvalues $\lambda_{1} \geq \lambda_{2} \geq \ldots \geq \lambda_{n}$.

The largest eigenvalue of $\mathbf{A}^{G}, \lambda_{1}$, is known as spectral radius, principal eigenvalue, or index of $G$. The spectral radius is associated with the structure of the graph. Its magnitude always decreases if an edge is removed from the graph (Brouwer and Haemers, 2011; Butler, 2008) and it is related to the average degree (the average number of incident edges to a vertex in the graph), denoted by $d e g_{a v e}$, and to the maximum degree, denoted by $d e g_{\text {max }}$, as follows (Brouwer and Haemers, 2011):

$$
\max \left\{d e g_{\text {ave }}, \sqrt{\left.\operatorname{deg_{\operatorname {max}}}\right\}} \leq \lambda_{1} \leq d e g_{\max }\right.
$$


In addition, the spectral radius is associated with the information flow of the graph. An interesting result shows that, for large values of $k$, the number of closed walks of length $k$ in $G$, given by the trace of the $k$ th order of the adjacency matrix $\mathbf{A}^{G}$, i.e, by trace of $\left(\mathbf{A}^{G}\right)^{k}$, can be approximated by $\lambda_{1}^{k}$ (Lovász, 1979, 1993). Also, Wang et al. (2003) show that the spectral radius captures propagation properties of a graph. The authors present a viral propagation model by showing that, under reasonable approximations, the epidemic threshold (the critical state beyond which infections become endemic) for arbitrary graphs is closely related to the spectral radius.

\subsubsection{Random Graph Models}

Any graph constructed by a random process is called random graph or random network. Random graph models refer to specific classes of random graphs or to probability spaces whose points are graphs on a fixed set of $n$ distinguishable vertices (Bollobás, 1998b).

The field of random graph models was introduced by Erdös and Rényi (Erdôs and Rényi, 1959; Erdos and Rényi, 1960), but many other plausible models have been introduced for studying structure and dynamics of observed complex networks. In the following, we define some of the most popular random graph models:

Erdös-Rényi random graph Erdös-Rényi random graphs (Erdôs and Rényi, 1959) are one of the most studied random graphs. Erdös and Rényi defined a random graph as $n$ labeled vertices where each pair of vertices $\left(V_{i}, V_{j}\right)$ is connected by an edge with a given probability $p$. The average number of edges in a Erdös-Rényi random graph is $\left(\begin{array}{l}n \\ 2\end{array}\right) p$.

Füredi and Komlós (1981) show that the spectral radius of an Erdös-Rényi random graph converges to $n p-2 p+1$. This relationship is illustrated in Figure 3.1A. Considering 10000 values for $p$ uniformly distributed between zero and one, we can note that the spectral radius of a Erdös-Rényi random graph with $n=300$ is always close to $300 p$. Füredi and Komlós (1981) also proved that the spectral radius is asymptotically normally distributed with mean $n p-2 p+1$ and variance $2 p(1-p)$. Finally, some results concerning the value of $n p$ were shown by Erdos and Rényi (1960). If $n p<1$, then the graph almost surely has no connected components of size larger than $O(\log (n))$. If $n p=1$, then the graph almost surely has a largest component whose size is of order $n^{2 / 3}$. And if $n p$ converges to a constant greater than 1 , then the graph almost surely has a unique component containing a positive fraction of the vertices.

Geometric random graph A geometric random graph (Gilbert, 1961) is a spatial network constructed by randomly placing $n$ vertices in some topological space $\mathrm{R}^{d}$ (e.g., a unit square if $d=2$ ) and connecting two vertices by an edge if their distance according to the $\ell_{d}$-norm (e.g., Euclidean norm if $d=2$ ) is smaller than a certain neighborhood radius $r$. Hence, geometric random graphs have a spatial element that is absent from other random graphs. For $d=2$, the expected degree of a vertex is $n \pi r^{2}$. 
Bordenave (2008) show that the spectral radius of the adjacency matrix is a function of the neighborhood radius. Specifically, the spectral radius of the adjacency matrix divided by $n$, i.e., $\mathbf{A}^{G} / n$, converges almost surely to $r^{d}$. Figure 3.1B illustrates the relationship between the spectral radius of $\mathbf{A}^{G}$ and the neighborhood radius $r$. Considering 10000 values for $r$ uniformly distributed between zero and one and $d=2$, we can note that the spectral radius of a geometric random graph of size 300 can be approximated by a non-linear monotonic function of $r$.

Regular random graph Regular random graphs started to be studied in the works of Bender (1974) and Bollobás (1980). A regular random graph is a graph in which all vertices have the same number of adjacent vertices, i.e., the same degree. A regular random graph with vertices of degree deg is called a random deg-regular graph.

Regular random graphs of degree at most two are well known: a 0-regular graph consists of only disconnected vertices; a 1-regular graph consists of disconnected edges; a 2-regular graph consists of one or more disconnected cycles of 3 vertices or infinite chains; a 3-regular graph is often called cubic graph and always has an even number of vertices (Tutte, 1959).

Alon (1986) showed that the spectral radius of a random deg-regular random graph is deg. This relationship is illustrated in Figure 3.1C. Considering 10000 values uniformly distributed between zero and one and defining deg as the integer part of these values multiplied by 10 , we can note that the spectral radius of deg-regular random graph is exactly deg.

Watts-Strogatz random graph The Watts-Strogatz random graph was proposed by Watts and Strogatz (1998). It is a random graph that interpolates between a regular lattice and an Erdös-Rényi random graph. This random graph presents small-world properties (short average path lengths, i.e., most vertices are not neighbors of one another but can be reached from every other vertex by a small number of steps) and a higher clustering coefficient (the number of triangles in the graph) than Erdös-Rényi random graphs.

Watts-Strogatz random graphs are determined by the parameters $n, n e i$, and $p_{w}$, which are, respectively, the number of vertices, the number of neighbors (mean degree), and the rewiring probability. They are generated by the following random procedure: (1) Construct a ring lattice with $n$ vertices, in which every vertex is connected to its first nei neighbors $\left(\frac{n e i}{2}\right.$ on either side); (2) For each vertex, reconnect, with probability $p_{w}$, the edge connecting its nearest neighbor in a clockwise sense to a vertex chosen uniformly at random over the entire ring; (3) Repeat this process by moving clockwise around the ring, considering each vertex in turn until one lap is completed; (4) Repeat steps 2 and 3, but considering now the edges connecting vertices to their second-nearest neighbors in a clockwise sense; (5) Continue this process, circulating around the ring and proceeding outward to more distant neighbors after each lap, until each edge in the original lattice has been considered once.

To the best of our knowledge, the spectral radius of a Watts-Strogatz random graph is not analytically defined, but Van Mieghem (2010) showed empirically that it is a function 
of $p_{w}$ and $n e i$. Considering 10000 values for $p_{w}$ uniformly distributed between zero and one and setting nei $=3$, we can note in Figure 3.1D that the spectral radii of the generated Watts-Strogatz random graphs (of size 300) can be approximated by a non-linear monotonic function of $p_{w}$.

Barabási-Albert random graph Proposed by Barabási and Albert (1999), it is a graph whose vertex degrees follow asymptotically a power-law distribution due to the preferential attachment property: the more connected a vertex is, the more likely it is to receive new edges. In general, Barabási-Albert random graphs have very few vertices with relatively high degrees, while the majority of vertices have low degrees. They can be generated by the following random procedure: (1) Start with a small number of $\left(n_{0}\right)$ vertices; (2) At every time-step, add a new vertex and connect it to $m_{1} \leq n_{0}$ different vertices already present in the system. The probability of the new vertex is connected to a vertex $V_{i}$ is proportional to the degree of $V_{i}$, denoted by $\operatorname{deg}\left(V_{i}\right)$, at the current time step, raised to the power of $p_{s}$, i.e., $P\left(V_{i}\right) \sim \operatorname{deg}\left(V_{i}\right)^{p_{s}}$. The scaling exponent $p_{s}$ indicates the order of the proportionality $\left(p_{s}=1\right.$ linear; $p_{s}=2$ quadratic and so on $)$.

After $t$ time-steps, a Barabási-Albert random graph will have $n=t+n_{0}$ vertices and $m_{1} t$ edges. Since, for any graph, the sum of the degrees of all vertices is equal to twice the number of edges, we have that the average degree of a Barabási-Albert random graph is constant and equal to $\left(2 m_{1} t\right) /\left(t+n_{0}\right)$ Barabási et al. (1999).

Let $k_{0}$ be the smallest degree. Dorogovtsev et al. (2003) showed that the spectral radius of the Barabási-Albert random graph is of the order of $k_{0}^{1 / 2} n^{1 / 2\left(p_{s}-1\right)}$. This relationship is illustrated in Figure 3.1E. Considering 10000 values uniformly distributed between zero and one and defining $p_{s}$ as these values multiplied by two, we can note that the spectral radius of a Barabási-Albert random graph of size 300 can be approximated by a non-linear monotonic function of $p_{s}$.

\subsubsection{Centrality Measures}

Next, we briefly describe the centrality measures of a vertex $V_{i}$ in a graph $G$ on a set $\boldsymbol{V}$ of $n$ vertices.

Degree The degree or valency of a vertex $V_{i} \in \boldsymbol{V}$ is the number of edges incident to $V_{i}$, which can be calculated from the adjacency matrix $\boldsymbol{A}^{G}$ of $G$, with entries $a_{i j}^{G}$ as follows (Bollobás, 1998a):

$$
\operatorname{deg}\left(V_{i}\right)=\sum_{j=1}^{n} a_{i j}^{G}
$$

Closeness centrality The closeness centrality of a vertex $V_{i}$ is a structural measure that reflects the average distance from $V_{i}$ to all others vertices in $\boldsymbol{V}$. Consider the geodesic distance $d_{g}\left(V_{i}, V_{j}\right)$ between the vertices $V_{i}, V_{j} \in \boldsymbol{V}$, given by the number of edges along the 

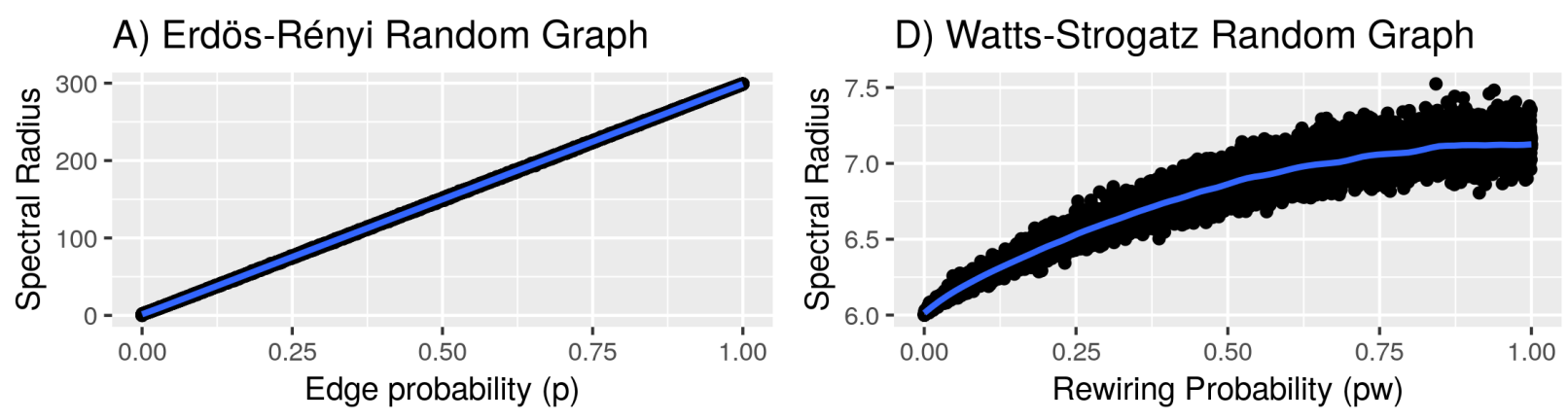

B) Geometric Random Graph

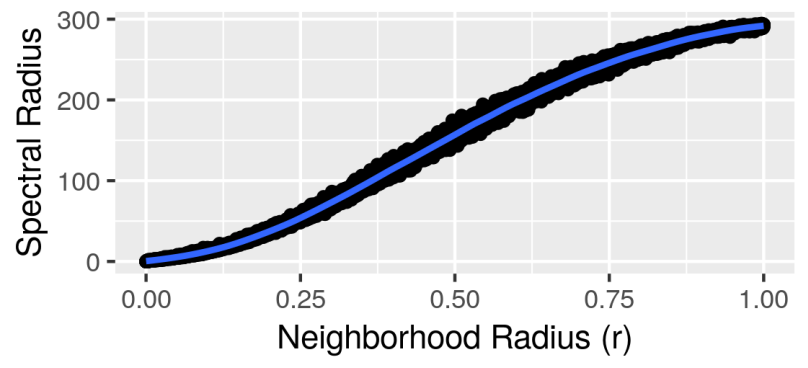

E) Barabási-Albert Random Graph

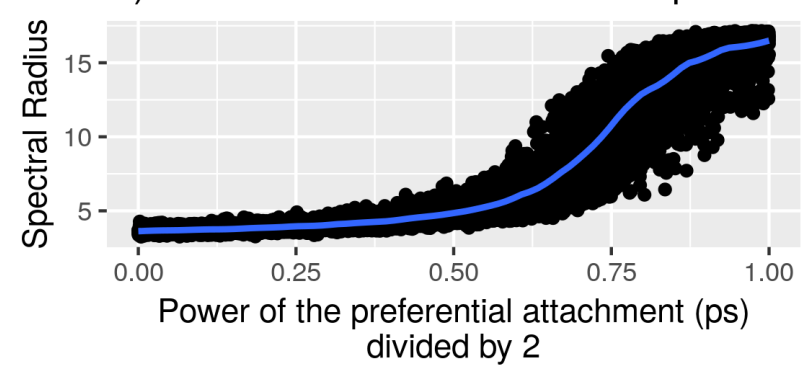

C) Regular Random Graph

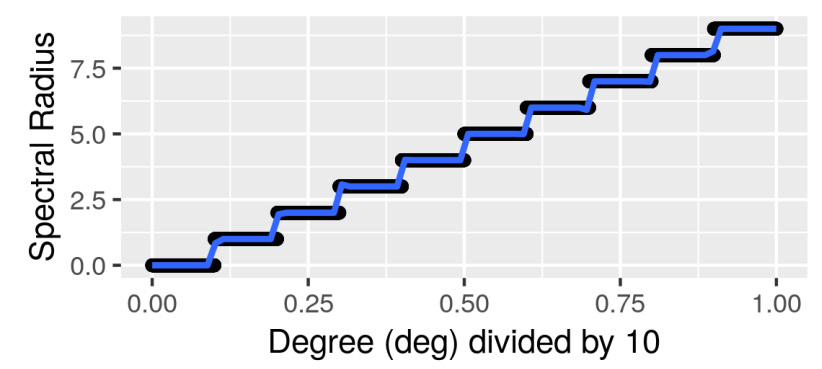

Figure 3.1: Functional relation between spectral radius and random graph model parameters estimated by 10000 replicates. In y-axis is shown the spectral radius of the random graph of size $n=300$ generated by using as model parameter the value shown in $x$-axis. (A) shows that the spectral radius of Erdös-Rényi random graphs with edge probability $p$ converges to $n p-2 p+1$. (B) shows that the spectral radius of geometric random graphs with $d=2$ and neighborhood radius $r$ is a function of $r^{2}$. (C) shows that the spectral radius of regular random graphs with degree deg is exactly deg. (D) shows that spectral radius of a Watts-Strogatz is a function of the rewiring probability $p_{w}$. (E) shows that the spectral radius of a Barabási-Albert random graph is a function of the power of the preferential attachment $p_{s}$.

shortest path between them. Also, consider the average geodesic distance for the vertex $V_{i}$, given by:

$$
d_{g m}\left(V_{i}\right)=\frac{1}{n-1} \sum_{V_{i} \neq V_{j}} d_{g}\left(V_{i}, V_{j}\right)
$$

Considering that a vertex with a small mean geodesic distance is important because it can reach the others vertices quickly, Bavelas (1950) defined the closeness centrality measure of a vertex $V_{i}$ as the multiplicative inverse of its average geodesic distance, i.e:

$$
\operatorname{closeness}\left(V_{i}\right)=\frac{1}{d_{g m}\left(V_{i}\right)}
$$


Betweenness centrality The betweenness centrality is an index for the number of times in which a vertex occurs on the shortest paths between other vertices. It was first formalized by Freeman (1977). The notion of importance of a vertex is based on its control over information passing between all other vertices. A vertex with a high betweenness centrality causes a major impact in the communication of the vertices if removed from the graph. Let $n_{s, t}^{(i)}$ be the number of geodesic paths (shortest paths) between $V_{s}$ and $V_{t}$ passing through $V_{i}$. Also, let $n_{s, t}$ be the number of geodesic paths between $V_{s}$ and $V_{t}$. Define the measure $w_{s, t}^{(i)}$ as

$$
w_{s, t}^{(i)}= \begin{cases}n_{s, t}^{(i)} / n_{s, t}, & \text { if } n_{s, t} \geq 0 \\ 0, & \text { otherwise }\end{cases}
$$

Then, the betweenness centrality of a vertex $V_{i}$ is given by:

$$
\operatorname{betweenness}\left(V_{i}\right)=\sum_{0<s, t \leq n} w_{s, t}^{(i)}
$$

Note that $s$ and $t$ can be equal to $i$. Thus, the index takes into account the information sent and received by $V_{i}$ itself.

Eigenvector centrality or eigencentrality The eigenvector centrality is a measure defined by Bonacich (1972) that reflects the notion of importance of a vertex not only by the number of neighbours (adjacent vertices) by also by the number of neighbours of its neighbours. Consider the adjacency matrix $\boldsymbol{A}^{G}$ of $G$, with entries $a_{i j}^{G}$. The eigenvector centrality of a vertex $V_{i}$ is given by

$$
\operatorname{evec}\left(V_{i}\right)=\frac{1}{\lambda} \sum_{V_{j} \in \mathcal{N}\left(V_{i}\right)} \operatorname{evec}\left(V_{j}\right)=\frac{1}{\lambda} \sum_{V_{j} \in V} a_{i, j}^{G} \operatorname{evec}\left(V_{j}\right)
$$

or, in matrix notation, by

$$
\operatorname{evec}(\mathbf{v})=\frac{1}{\lambda} \mathbf{A}^{\mathbf{G}} \operatorname{evec}(\mathbf{v})
$$

Usually this problem is solved by setting $\lambda$ as the largest eigenvalue of $\boldsymbol{A}^{G}$ and evec(v) as the associated eigenvector, called principal eigenvector. Thus, the eigenvector centrality of the vertex $V_{i}$ is defined as the entry $i$ of the principal eigenvector of $\boldsymbol{A}^{G}$. The use of the principal eigenvector, from an algebraic point of view, is justified by its association with the best approximation of rank 1 of the adjacency matrix. Furthermore, by the Perron-Frobenius theorem, the principal eigenvector of an irreducible matrix (as is the case of the adjacency matrix of an undirected connected graph) contains only positive entries.

\subsubsection{Granger Causality}

The definition of causality by Granger (1969) is based on the temporal precedence of the cause over the effect. We say that a process $x_{t}$ Granger causes a process $y_{t}$ if information on 
the past and present of $x_{t}$ helps to predict information on the future of $y_{t}$, after accounting for all other information relevant to the system.

In practice, the assumption that all information relevant to the system has been measured (called causal sufficiency) is rarely fulfilled. Thus, one may consider a less rigorous definition of Granger causality, in which only the available information is taken into account.

Lütkepohl (2005) formalized this idea as follows. Denote by $\Omega_{t}$ the set containing all the relevant information available up to and including period $t$. Denote by $\Sigma_{y}\left(h \mid \Omega_{t}\right)$ the forecast mean square error (MSE) of the $h$-step predictor (a predictor $h$ periods ahead) of $y_{t}$ at origin $t$, based on the information in $\Omega_{t}$. Also, denote by $\Sigma_{y}\left(h \mid \Omega_{t} \backslash\left\{x_{s} \mid s \leq t\right\}\right.$ the forecast MSE of the $h$-step predictor of $y_{t}$ at origin $t$, based on all information in $\Omega_{t}$ except for the information on the past and present of the $x_{t}$ process. The process $x_{t}$ is said to cause $y_{t}$ in Granger's sense (or that $x_{t}$ Granger-causes $y_{t}$ ) if there exists at least one forecast horizon $h>0$ such that

$$
\Sigma_{y}\left(h \mid \Omega_{t}\right)<\Sigma_{y}\left(h \mid \Omega_{t} \backslash\left\{x_{s} \mid s \leq t\right\}\right) .
$$

Thus, if $y_{t}$ can be predicted more efficiently if the information on the $x_{t}$ process is taken into account in addition to all other information, then $x_{t}$ Granger-causes $y_{t}$.

\subsubsection{Vector Autoregressive Model for Graphs}

In Sections 3.2.1.1 and 3.2.1.2, we discussed about important topological features of a random graph being captured by the parameters of its generating model and that, for several random graph models, it is known that the spectral radius is a function of the model parameters.

Thus, statistical analysis over graphs can be performed based solely on their spectral radii. Notably, Fujita et al. (2017) already used the spectral radius to construct a framework for identifying correlation between samples of graphs. They propose to test correlation between graphs by testing the Spearman's rank correlation between the samples of the respective spectral radii. The Spearman's rank correlation between two variables is equivalent to the Pearson correlation between the rank-transformed values of those two variables. The rank transformation allows the detection of monotonic relationships (linear or not), corrects for some departure from normality, increases the robustness to outliers, and eliminates any asymmetry, since all ranks are equally far apart from each other (Conover and Iman, 1981).

Inspired by Fujita et al. (2017), we introduce a new notion of Granger causality for graphs. By assuming that graphs are generated by models whose parameters are random variables, we define that a time series of graphs $y_{i, t}$ does not Granger cause another time series of graphs $y_{j, t}$ if the models parameters for $y_{i, t}$ does not Granger cause the model parameters for $y_{j, t}$.

VAR modeling is often used to identify Granger causality between stationary processes in the weak-sense, i.e., stochastic processes whose first and second moments are time invariant (Lütkepohl, 2005). VAR $(p)$ model is a system of linear equations in which the dependent 
variables are a function of an intercept and of the first $p$ lags of themselves and also of the other dependent variables. A desirable assumption for testing the coefficients of the VAR model is normality of the errors and, hence, normality of the observed variables. Since, in practice, the linearity and normality assumptions may be violated, our proposal is to identify Granger causality between time series of graphs by fitting VAR models on the time series of the ranks of the spectral radii (or average ranks in case of ties). This rank regression is a simple approach to perform a nonparametric test (Conover and Iman, 1981). In that case, the usual ordinary least square (OLS) estimator can be used to estimate the regression coefficients (Conover and Iman, 1981; Iman and Conover, 1979).

Specifically, let:

- $K$ be the number of time series of graphs;

- $p$ be the order of the model (number of time points in the past to be analyzed);

- $T$ be the time series length;

- $y_{i, t}$ be the rank of the spectral radius of the $i$ th graph at time $t$; and

- $u_{i, t}$ be the error term for the $i$ th graph, normally distributed, with zero mean and covariance matrix

$$
\boldsymbol{\Sigma}_{\mathbf{u}}=\left(\begin{array}{cccc}
\sigma_{1,1}^{2} & \sigma_{2,1} & \ldots & \sigma_{K, 1} \\
\sigma_{1,2} & \sigma_{2,2}^{2} & \ldots & \sigma_{K, 2} \\
\vdots & \vdots & \ddots & \vdots \\
\sigma_{1, K} & \sigma_{2, K} & \ldots & \sigma_{K, K}^{2}
\end{array}\right)
$$

Suppose that $y_{i, t}$, for $i=1, \ldots, K$, is a stable $\operatorname{VAR}(p)$ process, i.e., its reverse characteristic polynomial has no roots in or on the complex unit circle. This stability assumption can be verified by a unit root test, such as the augmented Dickey-Fuller (ADF) test (Dickey and Fuller, 1979) and, if it holds, then the process is stationarity, i.e., its first and second moments (mean and covariance) do not change with time.

Also, suppose that the $K$-dimensional vector of error terms $\boldsymbol{u}_{t}=\left\{u_{1, t}, \ldots, u_{K, t}\right\}$ is a standard innovation process, i.e., has zero mean $\left(\mathbb{E}\left(\boldsymbol{u}_{t}\right)=0\right)$ and finite variance $\left(\mathbb{E}\left(\boldsymbol{u}_{t} \boldsymbol{u}_{t}^{\prime}\right)=\right.$ $\left.\boldsymbol{\Sigma}_{\mathbf{u}}\right)$, the elements are serially uncorrelated $\left(\mathbb{E}\left(\boldsymbol{u}_{t} \boldsymbol{u}_{s}^{\prime}\right)=0\right.$ for $\left.s \neq t\right)$, and all fourth moments exist and are bounded. If it is normally distributed (i.e., is a Gaussian white noise), then the moment requirements are satisfied (Lütkepohl, 2005).

Note that the error terms $u_{i, t}$ are uncorrelated in time, but may be contemporaneously correlated. In other words, $\boldsymbol{\Sigma}_{\mathbf{u}}$ may not necessarily be an identity matrix.

Then, the $\operatorname{VAR}(p)$ model (VAR model of order $p$ ) for a $K$-dimensional process is the following system of equations in which each variable $y_{i, t}$ is regressed on a constant $\alpha_{i}$, on $p$ of its own lags (with coefficients $\beta_{i, i}^{l}$, for $l=1, \ldots, p$ ), and on $p$ lags of each of the other 
variables (with coefficients $\beta_{j, i}^{l}$, for $j \neq i, j=1, \ldots, K$, and $l=1, \ldots, p$ ):

$$
y_{i, t}=\alpha_{i}+\sum_{j=1}^{K} \sum_{l=1}^{p}\left(\beta_{j, i}^{l} y_{j, t-l}\right)+u_{i, t}, \quad \text { for } i=1, \ldots, K
$$

Or, equivalently,

$$
\left\{\begin{array}{l}
y_{1, t}=\alpha_{1}+\beta_{1,1}^{1} y_{1, t-1}+\ldots+\beta_{1,1}^{p} y_{1, t-p}+\ldots+\beta_{K, 1}^{1} y_{K, t-1}+\ldots+\beta_{K, 1}^{p} y_{K, t-p}+u_{1, t} \\
y_{2, t}=\alpha_{2}+\beta_{1,2}^{1} y_{1, t-1}+\ldots+\beta_{1,2}^{p} y_{1, t-p}+\ldots+\beta_{K, 2}^{1} y_{K, t-1}+\ldots+\beta_{K, 2}^{p} y_{K, t-p}+u_{2, t} \\
\vdots \\
y_{K, t}=\alpha_{K}+\beta_{1, K}^{1} y_{1, t-1}+\ldots+\beta_{1, K}^{p} y_{1, t-p}+\ldots+\beta_{K, K}^{1} y_{K, t-1}+\ldots+\beta_{K, K}^{p} y_{K, t-p}+u_{K, t}
\end{array}\right.
$$

To simplify and facilitate the estimation of the coefficients of this model, we will rewrite System 3.3 for $t=p+1, \ldots, T$ in the matrix form. Let

$$
\begin{aligned}
& \mathbf{Y}_{(T-p) \times K}=\left(\begin{array}{cccc}
y_{1, p+1} & y_{2, p+1} & \ldots & y_{K, p+1} \\
y_{1, p+2} & y_{2, p+2} & \ldots & y_{K, p+2} \\
\vdots & \vdots & \ddots & \vdots \\
y_{1, T} & y_{2, T} & \ldots & y_{K, T}
\end{array}\right) \\
& \mathbf{Z}_{(T-p) \times(K p+1)}=\left(\begin{array}{cccccccccc}
1 & y_{1, p} & y_{1, p-1} & \ldots & y_{1,1} & \ldots & y_{K, p} & y_{K, p-1} & \ldots & y_{K, 1} \\
1 & y_{1, p+1} & y_{1, p} & \ldots & y_{1,2} & \ldots & y_{K, p+1} & y_{K, p} & \ldots & y_{K, 2} \\
\vdots & \vdots & \ddots & \vdots & \ddots & \vdots & \vdots & \ddots & \vdots & \\
1 & y_{1, T-1} & y_{1, T-2} & \ldots & y_{1, T-p} & \ldots & y_{K, T-1} & y_{K, T-2} & \ldots & y_{K, T-p}
\end{array}\right), \\
& \boldsymbol{\beta}_{(K p+1) \times K}=\left(\begin{array}{cccc}
\alpha_{1} & \alpha_{2} & \ldots & \alpha_{K} \\
\beta_{1,1}^{1} & \beta_{1,2}^{1} & \ldots & \beta_{1, K}^{1} \\
\vdots & \vdots & \ddots & \vdots \\
\beta_{1,1}^{p} & \beta_{1,2}^{p} & \ldots & \beta_{1, K}^{p} \\
\vdots & \vdots & \ddots & \vdots \\
\beta_{K, 1}^{1} & \beta_{K, 2}^{1} & \ldots & \beta_{K, K}^{1} \\
\vdots & \vdots & \ddots & \vdots \\
\beta_{K, 1}^{p} & \beta_{K, 2}^{p} & \ldots & \beta_{K, K}^{p}
\end{array}\right)
\end{aligned}
$$

and

$$
\mathbf{u}_{(T-p) \times K}=\left(\begin{array}{cccc}
u_{1, p+1} & u_{2, p+1} & \ldots & u_{K, p+1} \\
u_{1, p+2} & u_{2, p+2} & \ldots & u_{K, p+2} \\
\vdots & \vdots & \ddots & \vdots \\
u_{1, T} & u_{2, T} & \ldots & u_{K, T}
\end{array}\right)
$$


Then, the $\operatorname{VAR}(p)$ model can be written in the matrix form as:

$$
\mathbf{Y}=\mathbf{Z} \boldsymbol{\beta}+\mathbf{u}
$$

The matrix $\boldsymbol{\beta}$ with all coefficients of the model, $\alpha_{i}$ and $\beta_{j, i}^{l}$, for $i, j=1, \ldots, K$, and $l=1, \ldots, p$, can be estimated by the usual Ordinary Least Squares (OLS) estimator as follows:

$$
\hat{\boldsymbol{\beta}}=\left(\mathbf{Z}^{\prime} \mathbf{Z}\right)^{-1} \mathbf{Z}^{\prime} \mathbf{Y}
$$

The consistency and asymptotic normality of the OLS estimates of $\boldsymbol{\beta}$ are ensured for stable $\operatorname{VAR}(p)$ processes with standard white noise residuals. Thus, $\hat{\boldsymbol{\beta}}$ is consistent and asymptotically normally distributed for stable Gaussian VAR $(p)$ processes (Lütkepohl, 2005).

The $(T-p) \times K$ matrix of residuals $\hat{\mathbf{u}}$ can be obtained as

$$
\hat{\mathbf{u}}=\mathbf{Y}-\mathbf{Z} \hat{\boldsymbol{\beta}}
$$

A consistent estimator of the $(K \times K)$ residual covariance matrix $\boldsymbol{\Sigma}_{\mathbf{u}}$ is

$$
\hat{\mathbf{\Sigma}}_{\mathbf{u}}=\frac{\hat{\mathbf{u}}^{\prime} \hat{\mathbf{u}}}{(T-p)-(K p+1)}
$$

where $\hat{\mathbf{u}}$ are the OLS residuals shown in Equation 3.6.

The lag order $p$ of a $\operatorname{VAR}(p)$ model is usually unknown. However it can be estimated using model selection criteria. The most common is the Akaike Information Criterion (AIC). Considering that a $K$-dimensional $\operatorname{VAR}(p)$ model has $p K$ lagged regressors and one intercept in each of the $K$ equations, the total number of regressors is $p K^{2}+K$. Thus, the AIC of a $\operatorname{VAR}(p)$ model is defined as (Lütkepohl, 2005):

$$
A I C(p)=\ln \left(\operatorname{det}\left(\frac{\hat{\mathbf{u}}^{\prime} \hat{\mathbf{u}}}{T-p}\right)\right)+\frac{2}{T}\left(p K^{2}+K\right) .
$$

\subsubsection{Granger Causality Tests}

By defining that graph $y_{i, t}$ does not Granger-cause graph $y_{j, t}$ if, and only if, $\beta_{i, j}^{l}=$ $0 \forall l=1, \ldots, p$, multiple restrictions have to be tested for determining the Granger causality significance $\left(H_{0}: \beta_{i, j}^{1}=\ldots=\beta_{i, j}^{p}=0\right)$. In this case, the Wald's test (Graybill, 1976), described in Section 3.2.3.1, or the parametric bootstrap procedure, described in Section 3.2.3.2, can be used.

\subsubsection{Wald's Test}

Suppose we are interested in testing the null hypothesis that $y_{i, t}$ does not Granger cause $y_{j, t}$. Then, we have to test if the coefficients $\beta_{i, j}^{1}, \ldots, \beta_{i, j}^{p}$ are all equal to zero. In other words, we have to test $H_{0}: \beta_{i, j}^{1}=\ldots=\beta_{i, j}^{p}=0$ versus the alternative hypothesis $H_{1}: \beta_{i, j}^{l} \neq 0$ for 
some $l=1, \ldots, p$.

To facilitate the notation, we will reformulate these hypotheses in matrix form.

Consider the $(K p+1) \times 1$ vector $\boldsymbol{\beta}_{\boldsymbol{j}}=\left(\alpha_{1}, \beta_{1, j}^{1}, \ldots, \beta_{1, j}^{p}, \ldots, \beta_{K, j}^{1}, \ldots, \beta_{K, j}^{p}\right)$, and also the $p \times(K p+1)$ matrix of contrasts $\mathbf{C}$ in which each row $l=1, \ldots, p$ contains the entry corresponding to $\beta_{i, j}^{l}$ equals to 1 and the others equal to 0 .

Then, the null hypothesis of Granger non-causality from $y_{i, t}$ to $y_{j, t}$ is $H_{0}: \boldsymbol{C}_{j}=\mathbf{0}_{p}$ and the alternative hypothesis is $H_{1}: \boldsymbol{C} \boldsymbol{\beta}_{j} \neq \mathbf{0}_{p}$.

Under the null hypothesis and under the assumption that $\hat{\boldsymbol{\beta}}$ is normally distributed, the Wald's test statistic

$$
W_{i \rightarrow j}=\frac{\left(\mathbf{C} \hat{\boldsymbol{\beta}}_{j}\right)^{\prime}\left(\mathbf{C}\left(\mathbf{Z}^{\prime} \mathbf{Z}\right)^{-1} \mathbf{C}^{\prime}\right)^{-1}\left(\mathbf{C} \hat{\boldsymbol{\beta}}_{j}\right)}{\hat{\sigma}_{j, j}} \stackrel{d}{\longrightarrow} \chi^{2}(p)
$$

i.e., $W_{i \rightarrow j}$ follows a $\chi^{2}$ distribution with $p$ (rank of the matrix $\mathbf{C}$, i.e., number of linear restrictions) degrees of freedom.

\subsubsection{Bootstrap Procedure}

When dealing with small sample size or when the assumption of Gaussian white noise for the error terms $u_{i, t}$ in the VAR model is violated, we suggest the following parametric bootstrap algorithm for assessing the significance of the Granger causality from $y_{i, t}$ to $y_{j, t}$ :

1. Fit the $\operatorname{VAR}(p)$ model (Equation 3.4) to the observed time series. Estimate the model coefficients (Equation 3.5), the residuals (Equation 3.6), and the Wald statistic $W_{i \rightarrow j}$ for assessing the Granger causality of interest (Equation 3.8);

2. Construct the matrix $\boldsymbol{\beta}_{0}$ of coefficients of the model under the null hypothesis by setting $\beta_{i j}^{l}=0 \forall l=1, \ldots, p$ and keeping the other coefficients as originally estimated in step 1 ;

3. Determine the fitted values $\hat{\boldsymbol{Y}}_{\mathbf{0}}=\boldsymbol{Z} \boldsymbol{\beta}_{0}$ of the model under the null hypothesis constructed in step 2;

4. Determine $\boldsymbol{u}^{*}$ by resampling with replacement the residuals obtained in step 1 ;

5. Determine the bootstrap multivariate time series $\boldsymbol{Y}^{*}=\hat{\boldsymbol{Y}}_{\mathbf{0}}+\boldsymbol{u}^{*}$;

6. Fit the $\operatorname{VAR}(p)$ model (Equation 3.4) to $\boldsymbol{Y}^{*}$. Estimate the bootstrap coefficients $\beta_{i, j}^{l *}$ and calculate the bootstrap Wald statistic $W_{i \rightarrow j}^{*}$;

7. Repeat steps 4 to 6 until the desired number of bootstrap samples is obtained;

8. Estimate the p-value by calculating the fraction of replicates of $W_{i \rightarrow j}^{*}$ on the bootstrap dataset, which is at least as large as the observed statistic $W_{i \rightarrow j}$ on the original dataset. 


\subsubsection{Implementation in the StatGraph R Package}

The proposed methods for identification of Granger causality between time series of graphs are implemented in R (Team et al., 2013) and are available as part of the statGraph $R$ package on CRAN at https://cran.r-project.org/package=statGraph.

\subsubsection{Simulation Study}

To evaluate the Type I error control and statistical power of the gVAR method, we conducted simulations studies with two different scenarios as described in the following. For all scenarios, the error terms $u_{i, t}$ are normally distributed, centered at zero, and weakly correlated, i.e., with $\operatorname{Cov}\left(u_{i, t}, u_{j, t}\right)=\left\{\begin{array}{ll}0.1, & \text { if } i \neq j \\ 1, & \text { if } i=j\end{array}\right.$.

Scenario 1 The simplest scenario, with time series of graphs $y_{1, t}$ Granger-causing time series $y_{2, t}$ :

$$
\left\{\begin{array}{l}
y_{1, t}=0.5 y_{1, t-1}+u_{1, t} \\
y_{2, t}=0.5 y_{1, t-1}+u_{2, t}
\end{array}\right.
$$

Scenario 2 A more complex scenario between 4 time series of graphs: $y_{1, t}$ is a common cause of $y_{2, t}$ and $y_{4, t} ; y_{2, t}$ is a collider (common effect of $y_{1, t}$ and $y_{4, t}$ ); $y_{4, t}$ is a mediator or intermediate factor between $y_{1, t}$ and $y_{2, t}$; and there is a feedback loop, $y_{2, t} \rightarrow y_{3, t} \rightarrow$ $y_{4, t} \rightarrow y_{2, t}:$

$$
\left\{\begin{array}{l}
y_{1, t}=u_{1, t} \\
y_{2, t}=0.5 y_{1, t-1}-0.5 y_{4, t-1}+u_{2, t} \\
y_{3, t}=0.5 y_{2, t-2}+u_{3, t} \\
y_{4, t}=0.5 y_{1, t-1}+0.5 y_{3, t-1}+u_{4, t} .
\end{array}\right.
$$

To simulate time series of random graphs whose parameters follow the aforementioned autoregressive processes, we first normalize the simulated values to the interval $(0 ; 1)$ by using the inverse-logit function and then use these values as parameters of the random model that generates the graph. The normalized simulated values are used as parameters for the Erdös-Rényi, geometric, regular, Barabási-Albert, and Watts-Strogatz random models as follows:

- Erdös-Rényi random graph: simulated values correspond to the probability $p$ of two vertices are connected;

- Geometric random graph: simulated values correspond to the neighborhood radius parameter, $r$;

- Regular random graph: the integer part of the simulated values, after being multiplied by 10 , corresponds to the degree parameter, deg; 
- Barabási-Albert random graph: simulated values, after being multiplied by 2, correspond to the power of the preferential attachment, $p_{s}$. The number $m_{1}$ of edges to be included at each iteration was fixed at one and the algorithm started with one vertex $\left(n_{0}=1\right)$;

- Watts-Strogatz random graph: simulated values correspond to the rewiring probability, $p_{w}$. The number of neighbors nei was fixed at three.

The graphs for each simulated scenario follow the same random graph model. Different numbers of vertices $(n=60,90,120,150,200,300)$ and lengths of time series ( $T=$ 25, 50, 75, 100) were considered. Each configuration was replicated 1000 times.

For each replicate, we applied the gVAR method for identifying Granger causality among the graphs. Since we do not want that errors due to the selection of the order of the VAR model contaminate the evaluation of the method, it was assumed known. However, in practice, this order can be chosen as the one that minimizes some model selection criterion, such $\mathrm{AIC}$ or BIC. In addition, to evaluate the performance of the spectral radius as a single feature of the graph, we also applied the method using the average (across all vertices of the graph) of the centrality measures described in Section 3.2.1.3: degree, eigenvector, betweenness, and closeness centrality. The significance of the VAR coefficients was assessed by the bootstrap procedure described in Section 3.2.3.2 with 1000 bootstrap samples. A significance level of $5 \%$ was used for the inferences.

The functions erdos.renyi.game, grg.game, k.regular.game, barabasi.game, and watts.strogatz.game of the igraph R package were used to generate, respectively, Erdös-Rényi, geometric, regular, Barabási-Albert, and Watts-Strogatz random graphs.

\subsubsection{Brain-Brain Interaction Between Two Professional Violinists}

To study the brain-brain interaction of two professional musicians playing a violin duo, we applied the gVAR method to the Functional Near Infrared Spectroscopy (fNIRS) data set, acquired from both violinists. Due to the robustness of fNIRS signals to head motion artifacts, brain experiments in freely-moving subjects can be reliably made. Also, fNIRS measurements are comparable to the blood-oxygenation-level-dependent (BOLD) measurements of functional magnetic resonance imaging (fMRI) for cortical regions.

\subsubsection{Dataset Description and Pre-Processing}

The complete description of the analyzed dataset can be found in Balardin et al. (2017). Briefly, the two violinists are 50 and 41 years old, males, right-handed, and have 40 and 28 years of regular practice, respectively. The fNIRS signals were acquired by a 23-channel NIRScout system, while they were playing an 30s excerpt of Antonio Vivaldi's Allegro from 
the Concerto No 1 in E Major, Op. 8, RV 269, "Spring". The fNIRS sampling rate was 7.81 $\mathrm{Hz}$ and the length of the signals is 219 .

The signal of each channel was temporally filtered with a band-pass filter $(0.01-0.2 \mathrm{~Hz})$, to remove cardiac and respiratory frequencies as well as very low frequency artifacts. Also, the signals were linearly detrended and standardized. The pre-processed signals are shown in Figure 3.2.

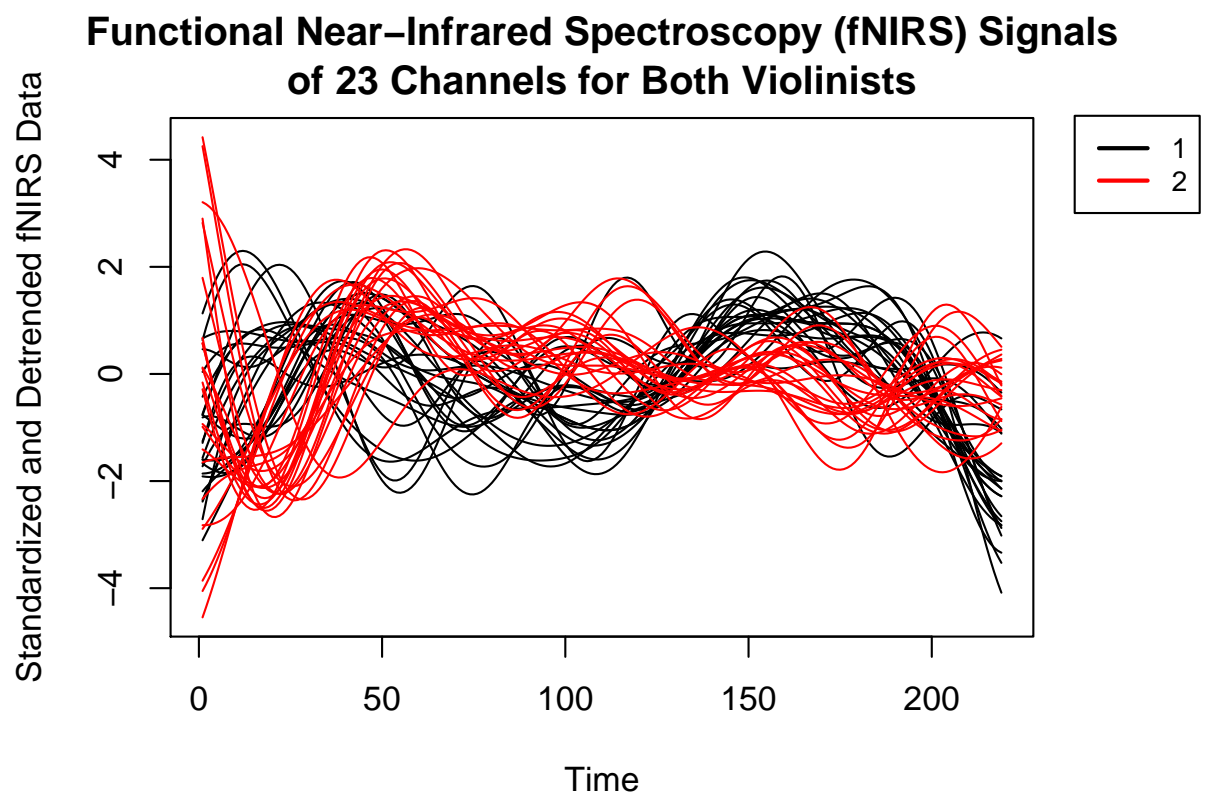

Figure 3.2: Pre-processed 23-channel fNIRS signals acquired simultaneously from two professional musicians when playing a violin duo. Time series of length 219 (approximately 28 seconds) are shown in black for the fist violinist and in red for the second violinist.

\subsubsection{Granger Causality Between Whole-Brain Functional Networks}

A common procedure for studying functional brain connectivity is to estimate a FBN as the Pearson correlation undirected graph in which the vertices are pre-defined brain regions of interest and the weights of the edges correspond to the Pearson correlation coefficient between the two time series in question.

Since we want to capture how these graphs evolve and interact over time using the same sampling rate of the observed time series, we estimate, for each violinist, a Pearson correlation undirected graph at each time point as follows.

Let $y_{1, t}$ and $y_{2, t}$ be the average BOLD time series for two regions of interest (ROIs). Then, consider the following smoothing functions:

- $\hat{\mu}_{y_{1, t}}$ and $\hat{\mu}_{y_{2, t}}$, representing the expected values of $y_{1, t}$ and $y_{2, t}$, estimated by spline interpolating (low pass filtering) the data points of $y_{1, t}$ and $y_{2, t}$, respectively;

- $\hat{\sigma}_{12, t}$, representing the covariance between $y_{1, t}$ and $y_{2, t}$, estimated by spline interpolating $\left[\left(y_{1, t}-\hat{\mu}_{y_{1, t}}\right)\left(y_{2, t}-\hat{\mu}_{y_{2, t}}\right)\right] ;$ and 
- $\hat{\sigma}_{1, t}^{2}$ and $\hat{\sigma}_{2, t}^{2}$, representing the variance of $y_{1, t}$ and $y_{2, t}$, estimated by spline interpolating, respectively, $\left(y_{1, t}-\hat{\mu}_{y_{1, t}}\right)^{2}$ and $\left(y_{2, t}-\hat{\mu}_{y_{2, t}}\right)^{2}$.

Then, the Pearson correlation between $y_{1, t}$ and $y_{2, t}$ at time $t$ is given by:

$$
\hat{\rho}_{12, t}=\frac{\hat{\sigma}_{12, t}}{\sqrt{\hat{\sigma}_{y_{1, t}}^{2}} \sqrt{\hat{\sigma}_{y_{2, t}}^{2}}}
$$

Due to oscillations at the edges, a problem known as polynomial wiggle or Runge's phenomenon (Runge, 1901), some values can be less than -1 or greater than 1 . In these cases, they were truncated to -1 and 1 , respectively.

Using this procedure, we estimate the pairwise Pearson correlations among the 23 fNIRs signals for each observed time point and for each violinist. Thus, we constructed two times series of FBNs with the same sampling rate of the fNIRs signals (219 time points). By assuming that the FBNs are realizations of random graphs, the Granger causality between both violinists can be identified by applying the gVAR method to these two time series of FBNs, with order of the VAR model selected by using the Akaike Information Criterion (AIC).

\subsubsection{Identifying Differential Connectivity Between Left and Right Brain Hemispheres in Autistic Subjects}

Here we aim at understanding how the functional networks of the left and right brain hemispheres affect each other over time in subjects with autism spectrum disorder (ASD) and typical controls, we applied the gVAR method to the Autism Brain Imaging Data Exchange I (ABIDE I) dataset (Craddock et al., 2013).

\subsubsection{ABIDE I Dataset}

The ABIDE I dataset is composed of resting state functional Magnetic Resonance Imaging (rs-fMRI), anatomical and phenotypic data of 1112 subjects (539 from subjects with ASD and 573 from typical controls, aged from 7 to 64 years), collected by 17 international sites. It is available at the ABIDE Consortium website (http://fcon_1000.projects.nitrc.org/ indi/abide/). The research performed at the ABIDE contributing sites complied with Health Insurance Portability and Accountability Act (HIPAA) guidelines and the 1000 Functional Connectomes Project/International Data-sharing Initiative protocols. All data distributed via the ABIDE website were fully anonymized in compliance with the HIPAA privacy rules, and no protected health information was included.

Since head movement during fMRI degrades data quality (Power et al., 2012), we considered for this application rs-fMRI and phenotypic data only from 737 subjects (308 subjects with ASD and 429 typical controls) for whom the mean framewise displacement (FD) is 
equal or less to 0.2. Also, we included as covariates the sites from where the data were collected, diagnostic status (ASD or typical control), sex, and age at scan. We had no missing data for these selected subjects.

\subsubsection{Data Pre-Processing}

The imaging data was first pre-processed by using the Neuro Bureau Athena pipeline, available at http://www.nitrc.org/plugins/mwiki/index.php/neurobureau:AthenaPipeline.

The pre-processing steps carried out for each subject is illustrated in Figure 3.3. First, we mapped the brain regions of interest (ROIs) according to the Anatomical Automatic Labeling (AAL) brain atlas Tzourio-Mazoyer et al. (2002) (Figure 3.3A). Out of the 116 brain regions included in the AAL atlas, we excluded 26 cerebellar regions (nine regions in each cerebellar hemisphere and eight regions in the vermis). Then, the remaining 90 regions were classified as belonging to the left or right brain hemisphere using as criterion the side containing the highest number of voxels (Figure 3.3B). We obtained 45 regions in each brain hemisphere. The average time series across all voxels within the ROI was considered as the region representative.

\subsubsection{Granger Causality Between Left and Right Brain Hemispheres}

Considering the fMRI data mapped and classified according to the left and the right brain hemispheres, we employed the same procedure shown in Section 3.2.6.2 to estimate, for each subject, two time series of Pearson correlation graphs: one representing the left brain and the other the right brain. Since the graphs are estimated at each time point of the original rsfMRI signals, the sampling rate of the time series of FBNs is preserved. This pre-processing step is illustrated in Figure 3.3C. Next, as shown in Figure 3.3D, by assuming that these graphs are realizations of random graphs, we used the gVAR method for investigating the Granger causality between both brain hemispheres. Since two causality directions have to be tested, two Wald's test statistics are obtained: one for assessing the significance of the Granger causality from the left to the right brain hemisphere $\left(W_{\text {Left-> Right }}\right)$, and the other for the right to the left brain hemisphere $\left(W_{\text {Right->Left }}\right)$. To check consistency of the results, the gVAR method was applied, for all subjects, with orders $p=4,5$, and 6 .

To assess differential Granger causality between the left and the right brain hemispheres in subjects with ASD, we performed linear regressions using the Box-Cox transformed Wald's test statistic as response variable, considering all subjects. The Box-Cox transformation makes the distribution of the Wald's test statistics approximately Gaussian. Since we have two Wald's test statistics, one for each causality direction, two separate linear regressions were performed. To control for variations among the sites that collected the data, we fitted a mixed model with two components of variance: $\gamma_{S I T E}$, for modeling the variability between sites, and $\varepsilon$, for capturing the residual variability. Both random effects are considered independent and normally distributed. The main effect for FD and the main and interaction 


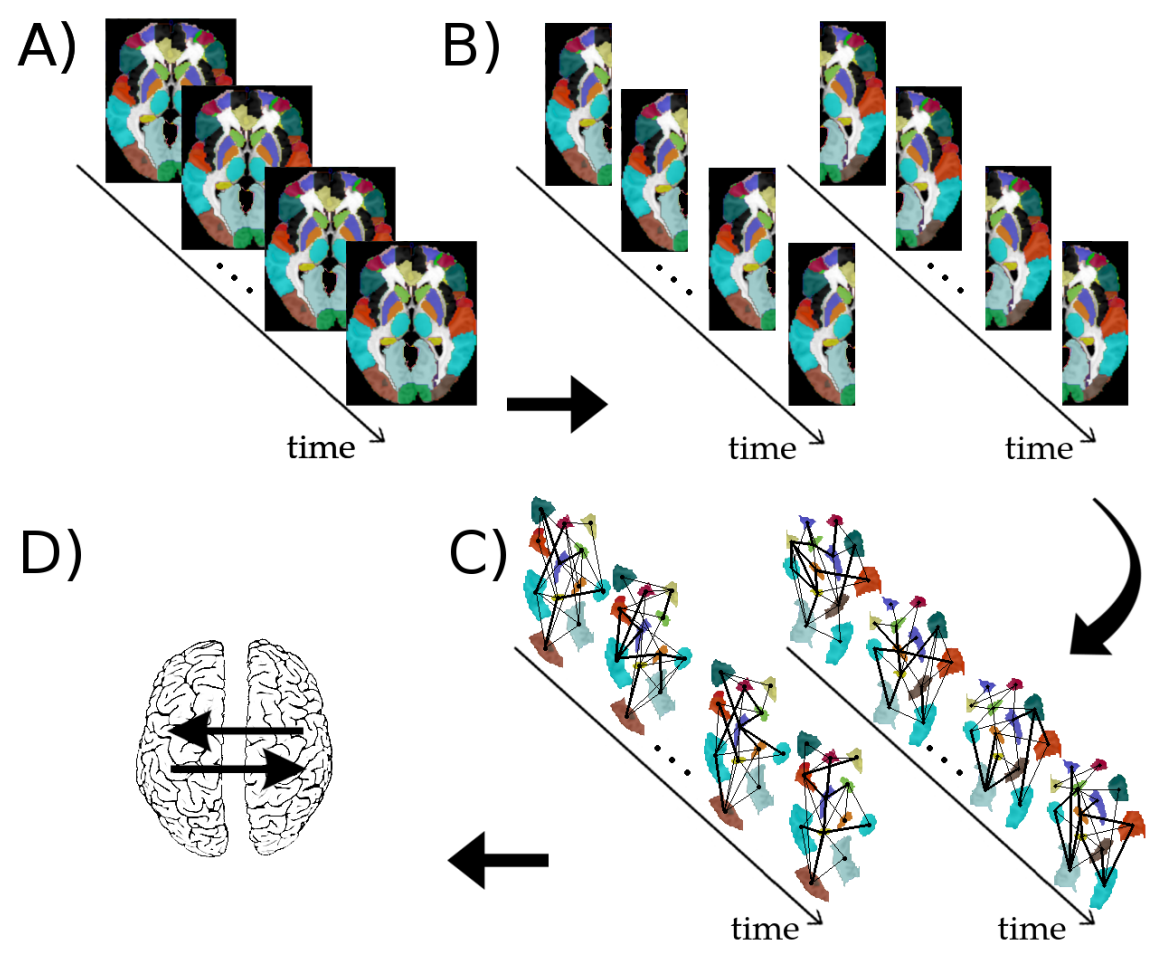

Figure 3.3: Preprocessing procedure for assessing subject-specific Granger causality between the functional networks for left and right brain hemispheres. A) rs-fMRI data segmented into 90 regions according to the $A A L$ atlas. B) Separation of the brain regions as belonging to the left or right hemisphere. C) time series of functional networks (graphs) for both left and right hemispheres. D) Identification of Granger causality between the left and right hemispheres.

effects for SEX (0: male, 1: female), AGE, and ASD (binary diagnostic) were evaluated.

Specifically, denote by $W_{\text {Right->Left }}$ the Wald's test statistic obtained for assessing the causality from the right to the left brain hemisphere, and consider an appropriate value for the parameter $\lambda$ of the Box-Cox transformation, for example, the one that maximizes the log-likelihood of the model with all covariates of interest. Also, let $\beta_{F D}, \beta_{S E X}, \beta_{A G E}$ and $\beta_{A S D}$ be the coefficients for the main effects of, respectively, FD, SEX, AGE, and diagnostic of ASD. And let $\beta_{S E X * A G E}, \beta_{S E X * A S D}, \beta_{A G E * A S D}$, and $\beta_{S E X * A G E * A S D}$ be the coefficients for the interaction effects between SEX, AGE, and diagnostic of ASD. Then, the following mixed model was considered:

$$
\begin{aligned}
\frac{W_{\text {Right->Left }}^{\lambda}-1}{\lambda} & =\alpha+\beta_{F D} F D+\beta_{S E X} S E X+\beta_{A G E} A G E+\beta_{A S D} A S D+ \\
& \beta_{S E X * A G E} S E X * A G E+\beta_{A G E * A S D} A G E * A S D+ \\
& \beta_{S E X * A S D} S E X * A S D+\beta_{S E X * A G E * A S D} S E X * A G E * A S D+\gamma_{S I T E}+\varepsilon
\end{aligned}
$$

For the Wald's test statistic obtained for assessing the causality from the left to the right brain hemisphere, we considered an analogous model. 


\subsection{Results and Discussions}

\subsubsection{Simulation Results}

We evaluate the accuracy of the gVAR method in the two scenarios described in Section 3.2.5. Particularly, the control of the false positive rate was assessed by the Granger noncausality from $y_{2, t}$ to $y_{1, t}$ in Scenario 1, while the statistical power was assessed by the true Granger causality from $y_{1, t}$ to $y_{2, t}$ in Scenario 1 and the five true Granger causalities in Scenario 2. For each scenario, we considered different lengths of the times series $(T=$ $25,50,75,100)$, different graph sizes $(n=60,90,120,150,200,300)$, and five random graph models, namely Erdös-Rényi, geometric, regular, Barabási-Albert, and Watts-Strogatz. Each configuration was repeated 1000 times. The number of bootstrap replicates was set to 1000 . A significance level of $5 \%$ was used for the inferences.

The results for the Granger non-causality from $y_{2, t}$ to $y_{1, t}$ in Scenario 1 are shown in Figure 3.4. The results obtained using the gVAR method, in which each graph is represented by the ranked spectral radius, are shown in the first columns of Figure 3.4. For all configurations considering Erdös-Rényi, geometric, regular, Barabási-Albert, and Watts-Strogatz random graph models, the gVAR method indeed controls the type I error. The subsequent columns of Figure 3.4 show the results of the gVAR method using as feature of the graph the average betweenness, closeness, eigenvector and degree centrality, respectively. The type I error was also controlled in these cases. For most configurations, particularly for large graphs and long time series, the false positive rate is below or equal to $5 \%$.

The results for the true Granger causality from $y_{1, t}$ to $y_{2, t}$ in Scenario 1 are shown in Figure 3.5. The first column shows the results using the spectral radius as feature of the graph and the subsequent columns show the results using the average betweenness, closeness, eigenvector and degree centrality, respectively. The degree centrality is always constant for Watts-Strogatz and Barabási-Albert random graphs, so this measure cannot be used as feature of the graphs. Particularly, for all simulated Watts-Strogatz random graphs, the degree of all vertices is the determined by the parameter nei (number of neighbours), that was fixed at three. So, for all Watts-Strogatz random graphs the average degree is three. In addition, for all simulated Barabási-Albert random graphs, the parameter $m_{1}$ (number of edges added at each time-step) was fixed at one and the algorithm started with one vertex $\left(n_{0}=1\right)$. Thus, as described in Section 3.2.1.2, the average degree is constant and, in this case, approximately two (exactly equals to $2(n-1) / n$ ). The "greener" is the heatmap, the greater is the proportion of rejected null hypotheses. In contrast, the "redder", the lower is the power. Results show that the power of the method based on the spectral radius is greater (or at least equivalent) than the ones based on these other centrality measures. Particularly, good accuracy for the regular, Watts-Strogatz, and Barabási-Albert random graphs could only be obtained by using the spectral radii of the graphs.

Results for the true Granger causalities in Scenario 2 are summarized in Figure 3.6. 


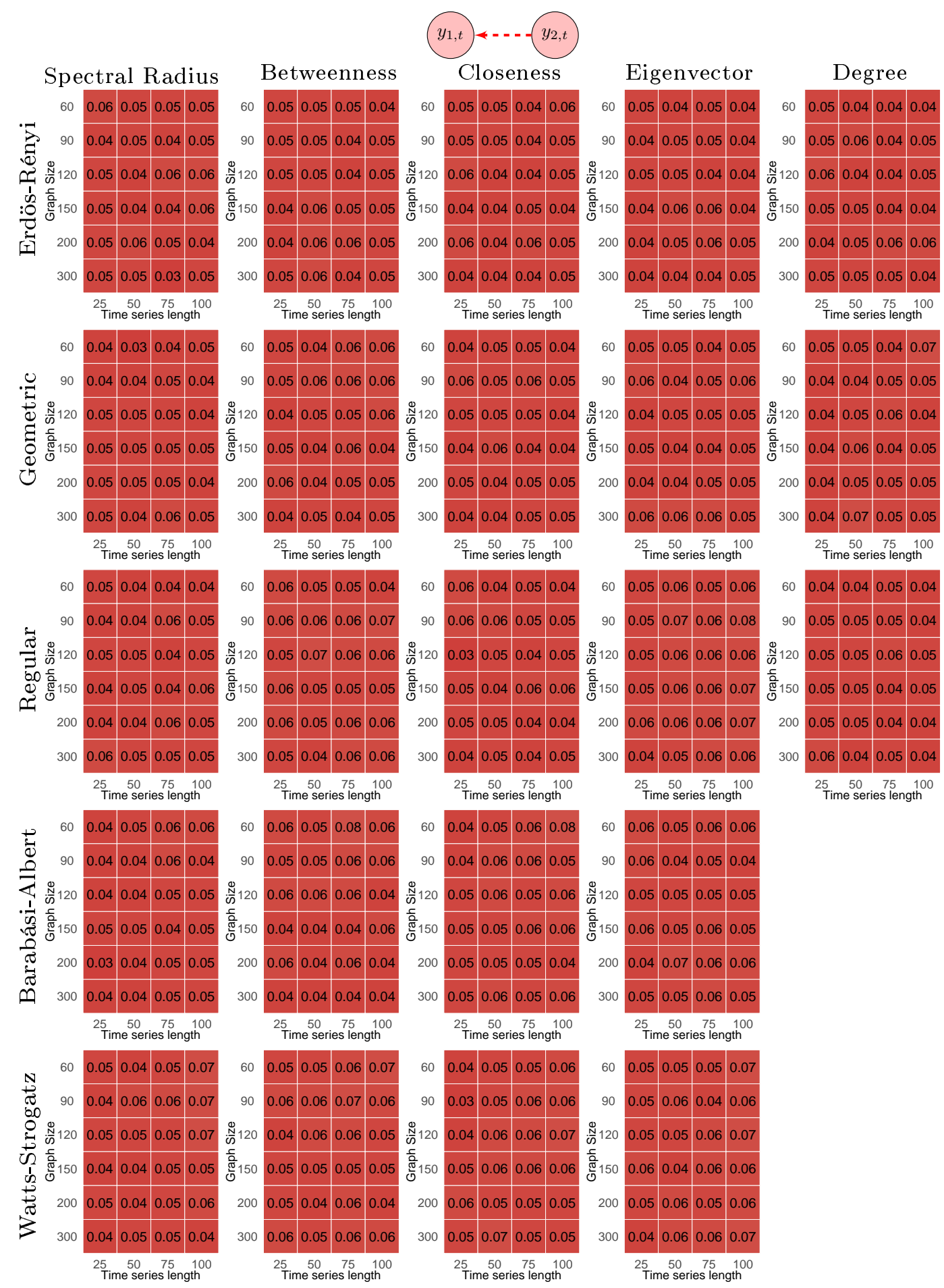

Figure 3.4: Heatmaps representing the proportion of rejected null hypotheses for the Granger noncausality from $y_{2}$ to $y_{1}$ in Scenario 1, at a significance level of 5\%. The first column of heatmaps show the results using the spectral radius as feature of the graph. The subsequent columns show, respectively, the results using the average betweenness, closeness, eigenvector and degree centralities as feature of the graph. The four columns of each heatmap correspond to the results obtained by varying the time series length $T=25,50,75,100$. The six rows correspond to the results obtained by varying the graphs sizes (number of vertices) $n=60,90,120,150,200,300$. The "greener" is the heatmap, the greater is the power. In contrast, the "redder", the lower is the proportion of rejected null hypotheses. Simulations were carried out by considering five random graph models, namely Erdös-Rényi, geometric, regular, Barabási-Albert, and Watts-Strogatz. Notice that all tests controlled effectively the type I error at a significance level of $5 \%$. 
We can notice that the greater is the time series length, the greater is the power of the test. For the Erdös-Rényi, geometric, and regular random graphs, the time series length is determinant to achieve accurate results. Good accuracy could be obtained for time series of 75 or more time points. It can be noticed for the Watts-Strogatz and Barabási-Albert random graph models that the loss of information due to the representation of the graphs by their spectral radii is higher than for other random graph models but that loss decreases as the size of the graph increases. Thus, the size of the graphs is another important factor affecting the accuracy of the gVAR method, i.e., the greater is the number of vertices of the graphs, the greater is the power of the method.

\subsubsection{Brain-Brain Interaction Between Two Professional Violinists}

After applying the pre-processing steps described in Section 3.2.6, we obtained the time series of the rank-transformed spectral radii of the graphs for the first and second violinist (denoted, respectively, by $y_{1, t}$ and $y_{2, t}$ ) are shown, respectively, in black and red on Figure 3.7. According to the ADF test, both time series are stable and, consequently, stationary (p-value $<0.01)$.

We identified at a significance level of $5 \%$ that the FBN of the first violinist Granger causes the FBN of the second violinist. Interestingly, the first violinist is older and a more experienced violinist. This result was obtained by fitting the gVAR method with order 10 (selected via AIC), which corresponds to a delay of 1.28 seconds. Thus, we can say that information 10 time points ahead on the structure and dynamics of the FBN of the second violinist can be predicted by information on the structure and dynamics of the FBN of the first violinist. The same Granger causality could be identified by fitting the gVAR method with orders 7 to 12 (delays of 0.89 to 1.53 seconds). The p-values showing the significance of Granger causality from the first to the second violinist are summarized in the first column of Table 3.1, and from the second to the first violinist, in the second column of Table 3.1.

By Figure 3.7, we notice that both time series are correlated and that the time series of the second violinist has a delay of approximately 10 time-points in relation to the time series of the first violinist.

Table 3.1: P-values for the $g V A R(p)$ method with orders $p=7, \ldots, 12$. Columns show, respectively, the selected order $p$, the p-value for the Granger causality from the first to the second violinist, and the p-value for the Granger causality from the second to the first violinist.

\begin{tabular}{ccc}
\hline \hline Order & $1 \rightarrow 2$ & $2 \rightarrow 1$ \\
\hline 7 & 0.002 & 0.258 \\
8 & 0.003 & 0.103 \\
9 & 0.002 & 0.057 \\
10 & 0.048 & 0.499 \\
11 & 0.038 & 0.767 \\
12 & 0.049 & 0.664 \\
\hline \hline
\end{tabular}




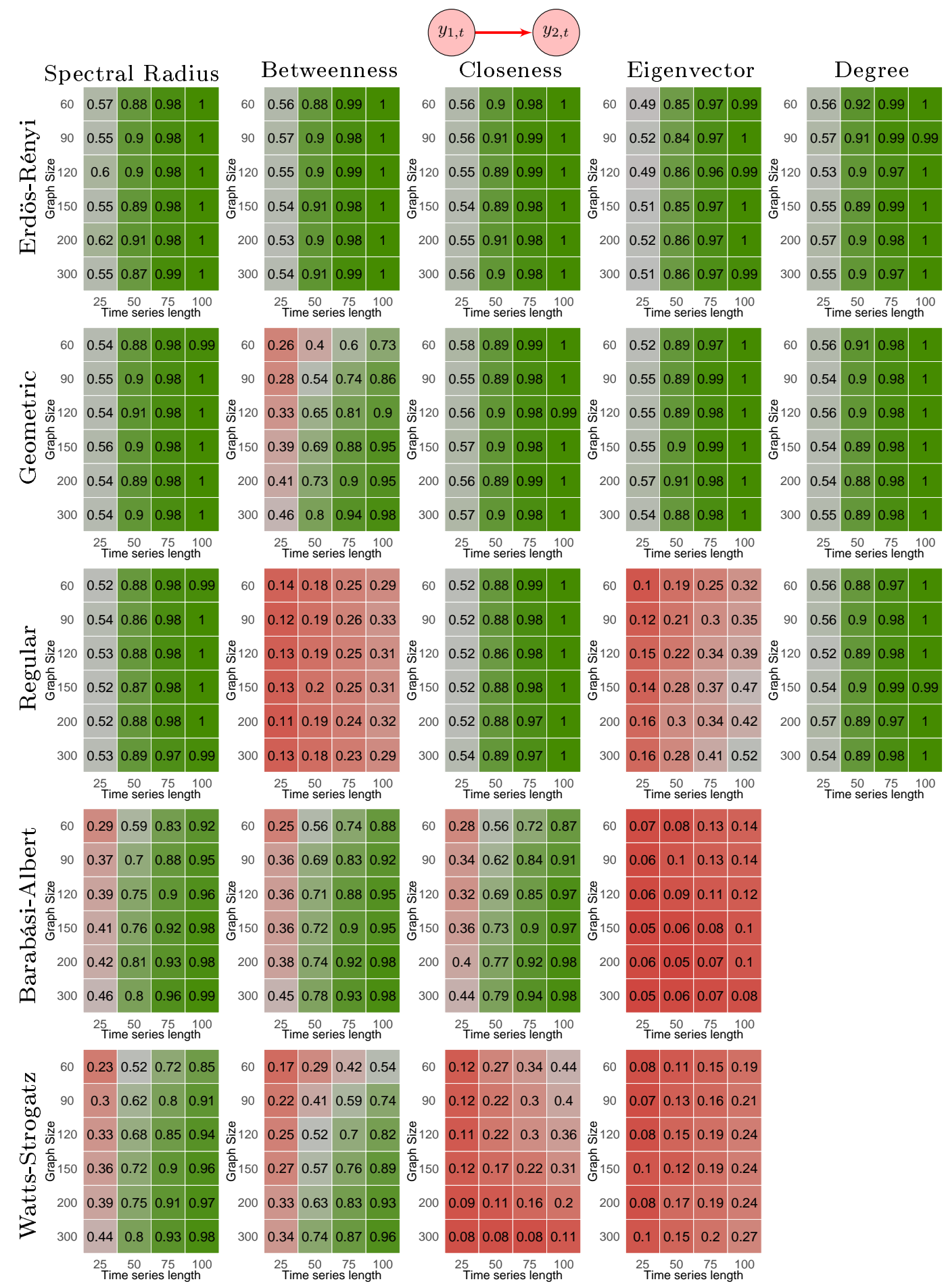

Figure 3.5: Heatmaps representing the proportion of rejected null hypotheses for the causality from $y_{1}$ to $y_{2}$ in Scenario 1, at a significance level of 5\%. The first column of heatmaps show the results using the spectral radius as feature of the graph. The subsequent columns show, respectively, the results using the average betweenness, closeness, eigenvector and degree centralities as feature of the graph. The four columns of each heatmap correspond to the results obtained by varying the time series length $T=25,50,75,100$. The six rows correspond to the results obtained by varying the graphs sizes (number of vertices) $n=60,90,120,150,200,300$. The "greener" is the heatmap, the greater is the power. In contrast, the "redder", the lower is the proportion of rejected null hypotheses. Simulations were carried out by considering five random graph models, namely Erdös-Rényi, geometric, regular, Barabási-Albert, and Watts-Strogatz. 


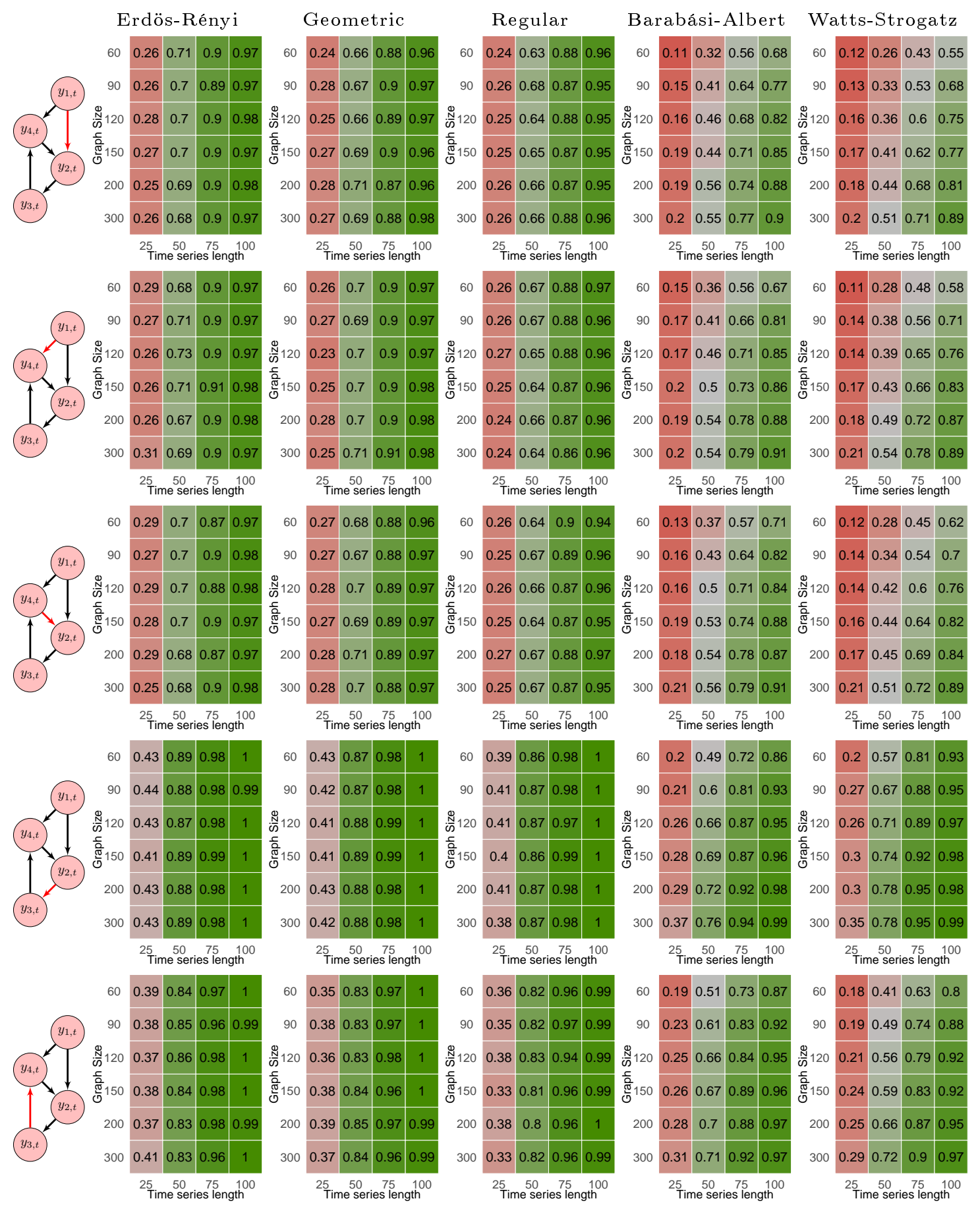

Figure 3.6: Heatmaps represent the proportion of rejected null hypotheses for the true Granger causalities in Scenario 2, at a significance level of 5\%. At the left, it is shown a schema of the true Granger causalities. Heatmaps represent the power of the test for the edge highlighted in red in the left schema. The four columns of each heatmap correspond to the results obtained by varying the time series length $T=25,50,75,100$. The six rows correspond to the results obtained by varying the graphs sizes (number of vertices) $n=60,90,120,150,200,300$. The "greener" is the heatmap, the greater is the power. In contrast, the "redder", the lower is the proportion of rejected null hypotheses. Simulations were carried out by considering five random graph models, namely Erdös-Rényi, geometric, regular, Barabási-Albert, and Watts-Strogatz. 
Spectral-Radius Signals for Both Violinists

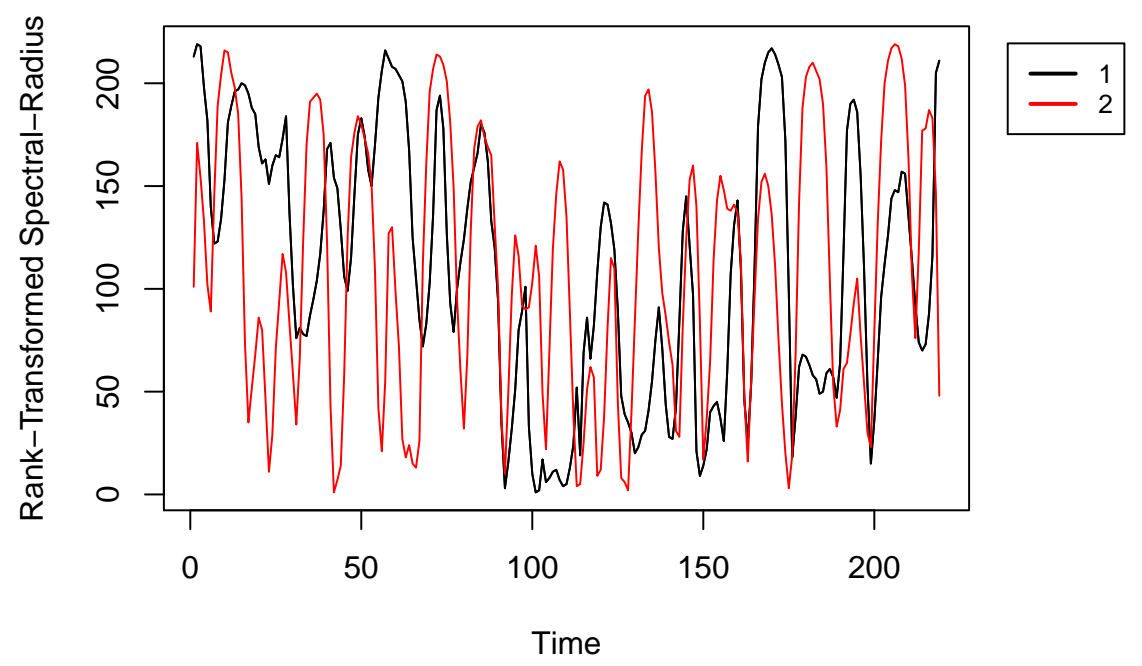

Figure 3.7: Time series of the rank-transformed spectral radii of the graphs for the first (in black) and the second (in red) violinist.

\subsubsection{Differential Connectivity Between Left and Right Brain Hemi- spheres in Autistic Subjects}

Considering a significance level of 5\%, the Granger causality from the left to the right brain hemispheres is not significantly different in autistic subjects and typical controls. By fitting the mixed model shown in 3.9 using as response variable the Box-Cox transformed Wald's test statistic $W_{\text {Left } \rightarrow \text { Right }}$, all effects were considered non-significant.

However, also at a significance level of $5 \%$, there is a differential Granger causality from the right to the left brain hemispheres in autistic subjects. By fitting the Model 3.9 using as response variable the Box-Cox transformed (using $\lambda=0.14$ ) Wald's test statistic $W_{\text {Right } \rightarrow \text { Left }}$, it was identified a significant interaction effect between AGE and diagnostic of ASD. Since the interaction effects $\beta_{S E X * A S D}$ and $\beta_{S E X * A G E * A S D}$ are non-significant, we remove them from the model. Thus, results will be presented considering the following model:

$$
\begin{aligned}
\frac{W_{\text {Right->Left }}^{\lambda}-1}{\lambda} & =\alpha+\beta_{F D} F D+\beta_{S E X} S E X+\beta_{A G E} A G E+\beta_{A S D} A S D+ \\
& \beta_{A G E * S E X} A G E * S E X+\beta_{A G E * A S D} A G E * A S D+\gamma_{S I T E}+\varepsilon
\end{aligned}
$$

To show consistency, we show in Tables 3.2, 3.3, and 3.4, the results for the regression coefficients in Model 3.10 when the response variable is, for all subjects, the Box-Cox transformed (using $\lambda=0.14$ ) Wald's test statistics $W_{\text {Right } \rightarrow \text { Left }}$ from the gVAR method with order $p$ fixed at, respectively, 4,5 , and 6 . The estimated variances for the random components $\gamma_{S I T E}$ and $\varepsilon$ are, respectively, 0.2626 and 1.0194 when $p=4,0.2433$ and 0.8824 when $p=5$, and 0.2150 and 0.7945 when $p=6$.

Figures 3.8-A and B illustrate the interaction effect between ASD and AGE, separately 
Table 3.2: Coefficient estimates, standard errors, and p-values of the fixed effects in Model 3.10, using Box-Cox transformed (using $\lambda=0.14$ ) Wald's test statistic $W_{\text {Right } \rightarrow \text { Left }}$ obtained from the gVAR method with order fixed at 4 .

\begin{tabular}{lccc}
\hline \hline Parameter & Estimate & Std. Error & P-value \\
\hline$\alpha$ & 2.365 & 0.229 & $<0.001$ \\
$\beta_{F D}$ & -1.932 & 0.955 & 0.043 \\
$\beta_{S E X}$ & 0.423 & 0.246 & 0.085 \\
$\beta_{A G E}$ & 0.013 & 0.007 & 0.094 \\
$\beta_{A S D}$ & 0.349 & 0.186 & 0.060 \\
$\boldsymbol{\beta}_{A G \boldsymbol{E} * \boldsymbol{A} \boldsymbol{S} \boldsymbol{D}}$ & $\mathbf{- 0 . 0 2 6}$ & $\mathbf{0 . 0 0 9}$ & $\mathbf{0 . 0 0 6}$ \\
$\beta_{A G E * S E X}$ & -0.021 & 0.013 & 0.123 \\
\hline \hline
\end{tabular}

Table 3.3: Coefficient estimates, standard errors, and p-values of the fixed effects in Model 3.10, using Box-Cox transformed (using $\lambda=0.14$ ) Wald's test statistic $W_{\text {Right } \rightarrow \text { Left }}$ obtained from the gVAR method with order fixed at 5.

\begin{tabular}{|c|c|c|c|}
\hline Parameter & Estimate & Std. Error & $\mathrm{P}$-value \\
\hline$\alpha$ & 2.527 & 0.216 & $<0.001$ \\
\hline$\beta_{F D}$ & -0.929 & 0.889 & 0.296 \\
\hline$\beta_{S E X}$ & 0.595 & 0.229 & 0.009 \\
\hline$\beta_{A G E}$ & 0.008 & 0.007 & 0.261 \\
\hline$\beta_{A S D}$ & 0.294 & 0.173 & 0.089 \\
\hline$\beta_{A G E * A S D}$ & -0.020 & 0.008 & 0.022 \\
\hline$\beta_{A G E * S E X}$ & -0.029 & 0.012 & 0.021 \\
\hline
\end{tabular}

Table 3.4: Coefficient estimates, standard errors, and p-values of the fixed effects in Model 3.10, using Box-Cox transformed (using $\lambda=0.14$ ) Wald's test statistic $W_{\text {Right } \rightarrow \text { Left }}$ obtained from the gVAR method with order fixed at 6 .

\begin{tabular}{lccc}
\hline \hline Parameter & Estimate & Std. Error & P-value \\
\hline$\alpha$ & 2.618 & 0.204 & $<0.001$ \\
$\beta_{F D}$ & -0.556 & 0.843 & 0.509 \\
$\beta_{S E X}$ & 0.703 & 0.217 & 0.001 \\
$\beta_{A G E}$ & 0.009 & 0.007 & 0.175 \\
$\beta_{A S D}$ & 0.224 & 0.164 & 0.171 \\
$\boldsymbol{\beta}_{\boldsymbol{A G E *} \boldsymbol{A S \boldsymbol { D }}}$ & $\mathbf{- 0 . 0 1 7}$ & $\mathbf{0 . 0 0 8}$ & $\mathbf{0 . 0 4 2}$ \\
$\beta_{A G E * S E X}$ & -0.033 & 0.012 & 0.005 \\
\hline \hline
\end{tabular}

for, respectively, male and female subjects, since there is also a significant effect of SEX.

We can note that the loss of functional connectivity from the right to the left brain hemisphere as age advances is significantly higher in subjects with autism. Specifically, considering the coefficient estimates shown in Table 3.3, the Granger causality (represented by the Box-Cox transformed Wald's test statistic $\left.W_{\text {Right } \rightarrow \text { Left }}\right)$ significantly decreases 0.012 $\left(\beta_{A G E * A S D}-\beta_{A G E}=0.020-0.008\right)$ each year in male subjects with autism, has a nonsignificant increase of $0.008\left(\beta_{A G E}\right)$ in male typical controls. Also, this same brain connectivity significantly decreases $0.041\left(\beta_{A G E * A S D}+\beta_{A G E * S E X}-\beta_{A G E}=0.020+0.029-0.008\right)$ 
each year in female subjects with autism, and only $0.021\left(\beta_{A G E * S E X}-\beta_{A G E}=0.029-0.008\right)$ each year in female typical controls.

We can also note in Figures 3.8-A and B, that autistic children have a higher Granger causality from the right to the left brain hemisphere than control children. This scenario starts to change at approximately 14 years old. Considering the Box-Cox transformed (using $\lambda=0.14)$ Wald's test statistic $W_{\text {Right } \rightarrow \text { Left }}$ obtained from the gVAR method with order fixed at 5, Figure 3.9 shows the (A) boxplots and (B) barplots of the means and 95\% confidence intervals, separated by the age ranges $(6,13],(13,15]$, and $(15,60]$ and by diagnostic status of ASD. According to a Welch's t-test, there is a differential Granger causality from the right to the left hemisphere in autistic subjects considering only females aged 6 to 13 years (mean for typical controls is 2.588, for subjects with ASD is 3.412, C.I. for the difference is $(-1.468,-0.180)$, $\mathrm{p}$-value $=0.014)$ and considering only males aged 16 to 60 years (mean for typical controls is 2.795 , for subjects with ASD is 2.486 , C.I. for the difference is $(0.075,0.541)$, p-value $=0.009)$.

\subsection{Conclusion}

Many biological problems can be plausibly modeled using random graphs. In neuroscience, for example, the brain connectivity is usually studied by representing meaningful anatomical or functional regions as networks (graphs). Considering that the structure and dynamics of the graphs may change over time, we proposed the gVAR method for identifying Granger causality among graphs. Specifically, Granger causality is identified by fitting a VAR model on the time series of the rank-transformed spectral-radii.

By simulation studies with Erdös-Rényi, geometric, regular, Barabási-Albert, and WattsStrogatz random graphs models, we showed that the gVAR method accurately infers causal relationships among random graphs and that the spectral radius is, compared to the average betweenness, closeness, eigenvector and degree centrality measures, the feature that captures more information regarding the model parameters and consequently is the most suitable feature for representing the graphs.

Interesting results could be drawn from this analysis, as demonstrated by the two applications to FBNs: one for studying brain-brain interaction between two musicians while playing a violin duo, and another for identifying differential connectivity between the FBNs of the left and right brain hemispheres in subjects with ASD.

The development of the gVAR method was inspired by the recent work of Fujita et al. (2017), which showed that it is possible to identify correlation between graphs using the spectral radius as a single feature of the graph. Since the spectral radius is a function of the parameters of several random graphs, it captures some of the topological features induced by the model parameters.

Based on this idea, many statistical methods can be easily extended or developed for 
A)

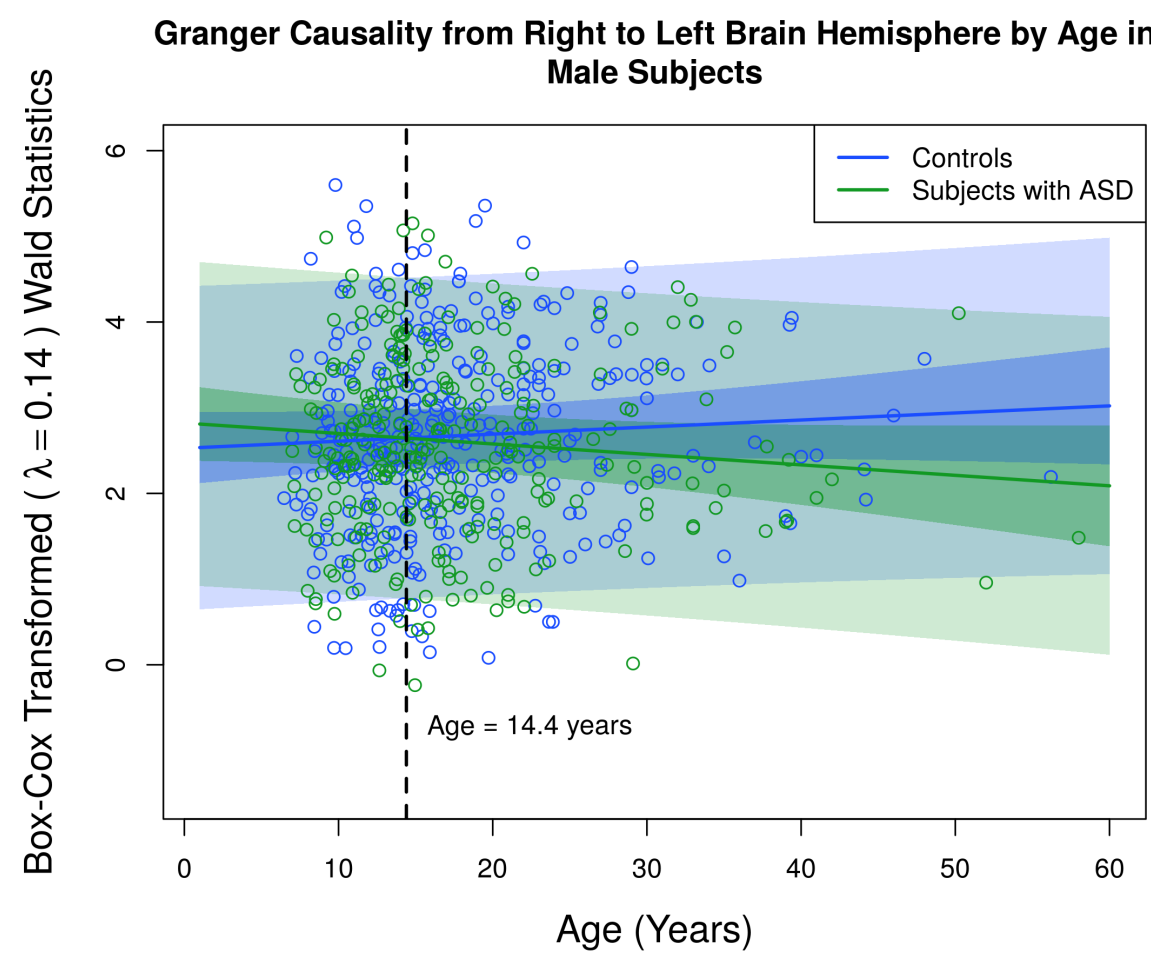

B)

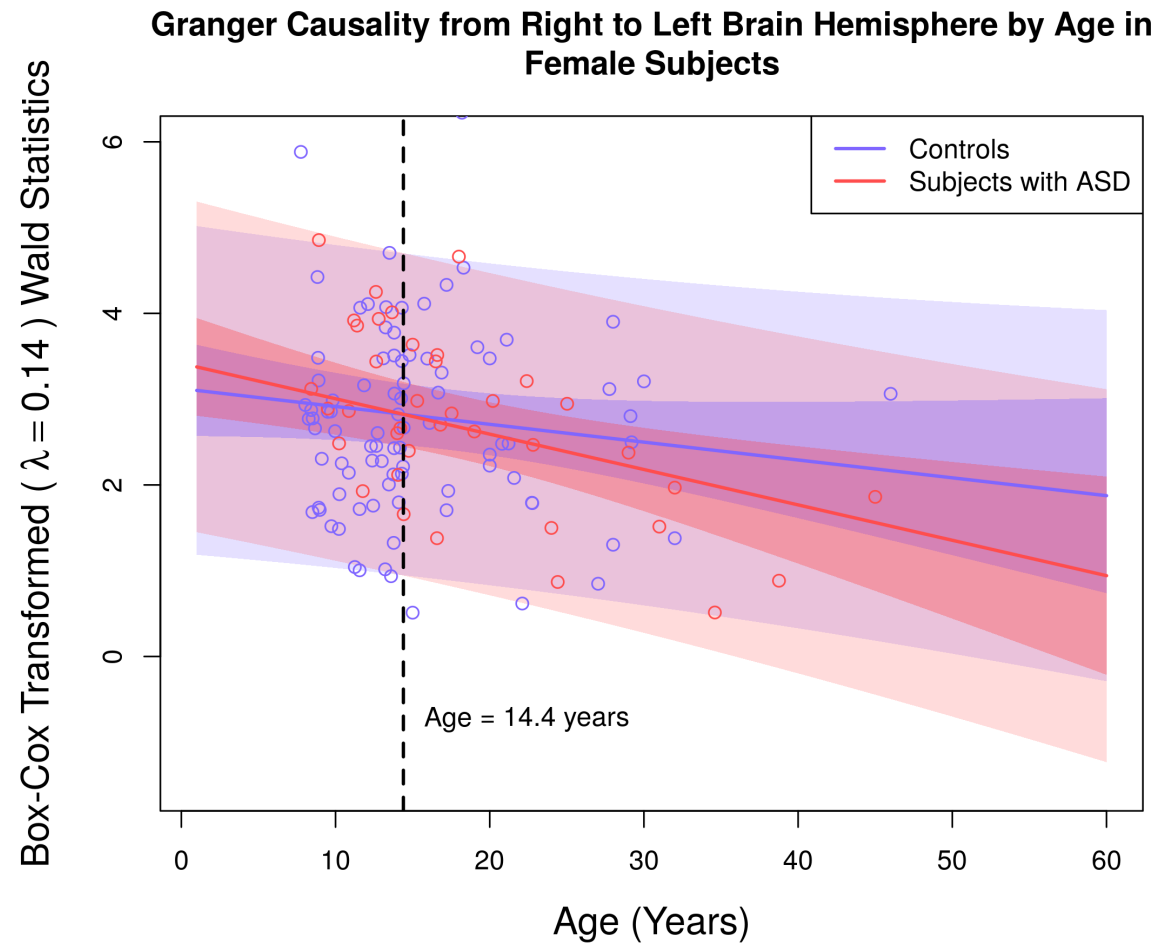

Figure 3.8: Observed values of the Box-Cox transformed (using $\lambda=0.14$ ) Wald's test statistic $W_{\text {Right } \rightarrow \text { Left }}$ obtained from the gVAR method with order fixed at 5, (A) for male typical controls (dots in blue) and male subjects with ASD (dots in green), and (B)for female typical controls (dots in violet) and female subjects with ASD (dots in pink). The curves were fitted considering Model 3.10 with $F D=0$ and ages from 0 to 60 years. Narrow shaded areas represent the $95 \%$ prediction intervals considering only the standard deviation of the random effect $\gamma_{S I T E}$ and the wide shaded areas represent the $95 \%$ prediction intervals considering also the standard deviation of the error $\varepsilon$. 
A) Boxplot for the Granger Causality from Right to Left Brain Hemisphere

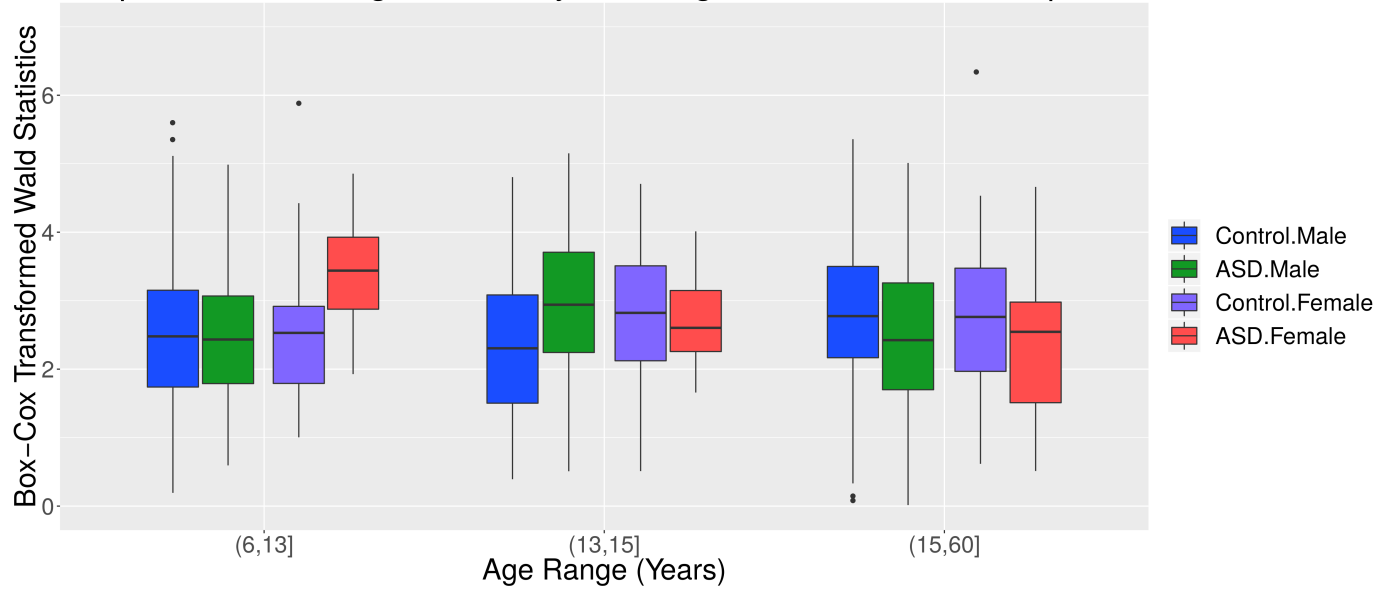

B)

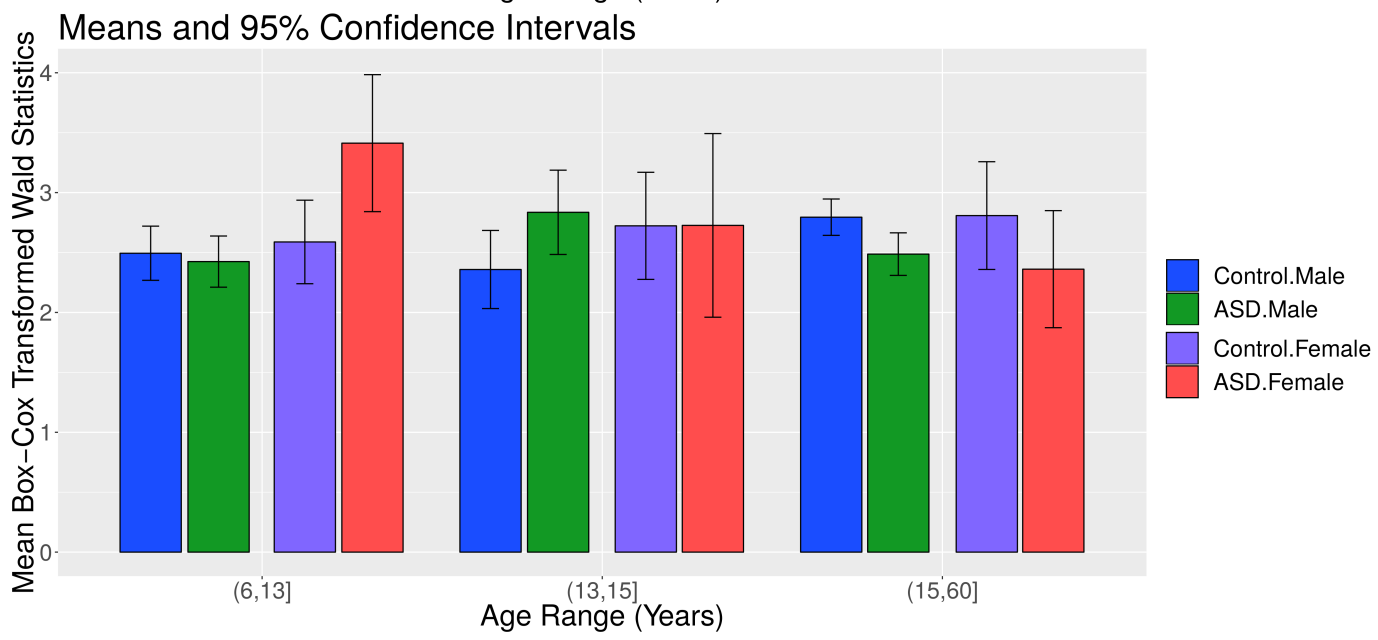

Figure 3.9: (A) boxplot and (B) barplot, by age range and diagnostic status of ASD, of the means and $95 \%$ confidence intervals of the Box-Cox transformed (using $\lambda=0.14$ ) Wald's test statistic $W_{\text {Right } \rightarrow \text { Left }}$ obtained from the $g V A R$ method with order fixed at five.

samples of graphs and inferences can be made regardless of whether the models that generate the observed graphs and their parameters are known or not.

However, a limitation concerning the interpretation of the results need to be acknowledged. If it was identified that a time series of graphs $y_{1, t}$ Granger causes another time series of graphs $y_{2, t}$, than we can only say that information on past topological properties of $y_{1, t}$ can predict topological properties of $y_{2, t}$. Since we do not know exactly which topological properties are captured by the spectral radius, we do not know exactly what changed in $y_{1, t}$ and was transmitted to the $y_{2, t}$. Thus, it may be difficult to extrapolate the conclusions to give a practical or biological meaning to them. Thus, more studies are needed to better understand the properties of the graph that are encoded in the spectral radius. In addition, further studies should be conducted to evaluate the impact of including other features of the graph in the analysis, such as the second largest eigenvalue of the graph or even all the spectral density.

For neuroscience research, a characterization of the properties of brain networks in the context of random graphs may be useful in understanding the information that flows through 
the brain networks and thus intervening in neurological disorders and neurodegenerative diseases. 


\section{Chapter 4}

\section{Conclusions}

Structure learning and causal inference in biological systems may aid in better understanding the mechanisms behind multifactorial diseases and traits and, consequently, in developing treatments that reduce the effects of the risk factors.

However, causal learning from purely observational data is a very difficult problem and results may be unreliable if assumptions are not met or if confounding factors are not considered in the analysis. These confounders induce dependencies among the variables and may be common causes of variables or grouping factors, such as genetic relatedness, temporal, and spatial patterns. Thus, causal structure learning and causal inference from high-dimensional data with complex dependence structure is an even harder problem.

The development of structure learning techniques from observational data is an ongoing research topic and there is still plenty of room for improvement.

In this work, we propose structure learning methods that take into account common issues in the fields of genetics and neuroscience.

In genetics, causality studies help to understand how phenotypic variables are related to each other, but few methods exploit the possible underlying structures of the associations.

Polygenic mixed models have been widely used for modeling family data in genomewide association studies (GWAS), providing a decomposition of the phenotypic variability according to genetic and environmental influences.

In Chapter 2, considering Gaussian family data, we used univariate polygenic mixed models to decompose phenotypic covariability according to genetic and environmental influences. In this approach, we proposed a correction for the confounding in the predicted random effects and in the conditional residuals, when estimated via ML or REML estimator. Thus, we could derive unconfounded estimators for the genetic and environmental partial correlations coefficients as well as tests for assessing their significance. By using the proposed zero partial correlation tests, we developed methods that learn the structure of undirected and directed PGMs among multiple phenotypes and also provide an unconfounded decomposition of these structures into genetic and environmental PGMs.

We evaluated the methods by simulation studies and by an application to GAW 13 
simulated data, that mimics some of the features of the real Framingham Heart Study data. We also applied the methods to the Baependi Heart Study data to learn the total, genetic, and environmental networks describing the relationships among the metabolic syndrome phenotypes.

The associations in the genetic PGM reflect the between-family covariability, while the association in the environmental PGM reflect the within-family covariability. Thus, if two phenotypes are, for example, negatively associated in the genetic PGM, we can say that if the average value for one phenotype is high in a family, then probably the average value for the other phenotype is low. Now, regardless of the genetic association, if these same two phenotypes are, for example, positively associated in the environmental PGM, we can say that if the level of one phenotype increase for an individual (conditional to its family) then the level of the other phenotype will probably also increase. The genetic association can be explained by a pleiotropic effect of one or more genes on both connected phenotypes. In contrast, the environmental association can be explained by subject-specific factors or by external factors to which the individual was exposed.

By considering a more discriminant decomposition of the covariance matrix in the polygenic mixed model, it is possible to improve inference of the causal effects as well as to elucidate better the structure of the associations. For example, similarities between relatives may be due to heritable genetic effects, but also to shared (household) environmental conditions, to interaction effects between genes and environmental factors (i.e., genetic effects vary according environmental conditions), or even to sex- or age-specific genetic effects. Thus, as future work, we intend to extend the methods by including random effects that model these other variability factors.

In neuroscience, the study of functional brain networks involves the analysis of highdimensional data with a complex dependence structure among the variables. Particularly, the brain regions are constantly interacting with each other, exhibiting a connectivity pattern that changes over time.

Thus, to study the interdependence structure among the graphs and to understand how the connectivity pattern of a graph affects the connectivity pattern of other graphs, we introduce in Chapter 3 a new notion of Granger causality for graphs. By assuming that the graphs are generated by random graph models and that the connectivity pattern (encoded in the structure of graph) is determined by the model parameters, we propose a definition of Granger causality between time series of graphs that is based on predictability and temporal precedence between the parameters that generate the graph. From the spectral graph theory, it is known that the spectral radius is a function of the parameters of several random graphs models. Consequently, the spectral radius encodes the connectivity pattern of the graph. Thus, we propose a method that identifies Granger causality among time series of graphs by fitting a VAR model to the time series of the rank-transformed spectral radii. Simulation studies showed that the spectral radius is indeed a good feature of the graphs for identifying Granger causality. 
We obtained interesting results with two applications of the proposed method in neuroscience. We studied brain-brain interaction between two musicians while playing violin duo and identified that the older and more experienced violinist Granger causes the other violinist considering a lag of approximately 1s. In addition, we identified that the connectivity from the right brain hemisphere to the left brain hemisphere changes with age differently in typical controls and autistic subjects. More specifically, this connectivity decreases more rapidly in autistic subjects.

The idea of capturing structural characteristics of the graphs by using their respective spectral radii was proven to be useful for measuring correlation between graphs by Fujita et al. (2017) and now for identifying Granger causality between time series of graphs. As a single feature is used to represent the graphs, many statistical approaches can be easily developed based on the same idea.

Other approaches could be proposed to perform causal analysis over graphs. For example, we could consider each brain region as a variable of interest (dozens, perhaps hundreds, of brain regions), whose BOLD measurement would be observed at several time points for each individual. Since measurements are obtained following a hierarchical structure, we could use an approach based on linear mixed models, similar to that developed in Chapter 2 , to learn the causal network among brain regions. Specifically, the partial correlation coefficients could be estimated by linear mixed models with a random effect for modeling the temporal dependence among the observations (usually used for modeling longitudinal data) and the total graph could be decomposed into a temporal and a residual graph. The causal relationships in the temporal graph could be interpreted as causalities induced by a temporal dependence, similar to that for the Granger causality. Another analysis that we could have perform is to consider the left and the right hemispheres as variables of interest, whose BOLD measurements are observed in many paired brain regions at several time points for each individual. In this case, we could obtain the causal relationships between the left and the right hemispheres by fitting mixed models with a random effect for modeling temporal dependence among the observations and another for modeling spatial dependence among the brain regions. Random effects for modeling interaction effects could also be considered. Thus, we could decompose the causal relations according to temporal and spacial influences. Note that in these both analyses, the causal network is learned for the population.

Although theses approaches can be considered more sophisticated, they are less flexible and computationally more difficult than the solution suggested in Chapter 3. For example, it would not be possible to use that approach to learn causal networks in studies with few replicates, as in the study of the brain-brain interaction between two violinists, or if the replicates are not hierarchically organized. In addition, it would be computationally expensive to obtain a causal network with dozens of vertices and multiple random factors. Regularization and dimension reduction approaches would be need to solve these high-dimensional problems.

The methods proposed in Chapter 2 model the within-cluster dependencies based on 
distributional properties of the data, while the method proposed in Chapter 3 model the within-cluster dependencies based on topological properties of the graphs (those captured by the spectral radius) that describe their relationships. Thus, an interesting direction for future work would be to develop a structure leaning algorithm for complex and high-dimensional data by combining mixed models with many components of the covariance and dimensionality reduction techniques based on spectral properties of random graphs. 


\section{Bibliography}

Allison et al.(1998) David B Allison, Bonnie Thiel, Pamela St Jean, Robert C Elston, Ming C Infante and Nicholas J Schork. Multiple phenotype modeling in gene-mapping studies of quantitative traits: power advantages. The American Journal of Human Genetics, 63(4):1190-1201. Cited in page 37

Almasy and Blangero(1998) Laura Almasy and John Blangero. Multipoint quantitativetrait linkage analysis in general pedigrees. The American Journal of Human Genetics, 62 (5):1198-1211. Cited in page 2, 6, 18

Alon(1986) Noga Alon. Eigenvalues and expanders. Combinatorica, 6(2):83-96. Cited in page $3,42,45$

Amos(1994) Christopher I Amos. Robust variance-components approach for assessing genetic linkage in pedigrees. American journal of human genetics, 54(3):535. Cited in page 2, 6,18

Amos et al.(2001) Christopher I Amos, Mariza De Andrade and Dakai K Zhu. Comparison of multivariate tests for genetic linkage. Human Heredity, 51(3):133-144. Cited in page 37

Anderson et al.(1958) Theodore Wilbur Anderson, Theodore Wilbur Anderson, Theodore Wilbur Anderson, Theodore Wilbur Anderson and Etats-Unis Mathématicien. An introduction to multivariate statistical analysis, volume 2. Wiley New York. Cited in page 13

Balardin et al.(2017) Joana B. Balardin, Guilherme A. Zimeo Morais, Rogério A. Furucho, Lucas Trambaiolli, Patricia Vanzella, Claudinei Biazoli and João R. Sato. Imaging brain function with functional near-infrared spectroscopy in unconstrained environments. Frontiers in Human Neuroscience, 11:258. Cited in page 43, 55

Barabási and Albert(1999) Albert-László Barabási and Réka Albert. Emergence of scaling in random networks. science, 286(5439):509-512. Cited in page 42, 46

Barabási et al.(1999) Albert-László Barabási, Réka Albert and Hawoong Jeong. Meanfield theory for scale-free random networks. Physica A: Statistical Mechanics and its Applications, 272(1-2):173-187. Cited in page 46

Bavelas(1950) Alex Bavelas. Communication patterns in task-oriented groups. Journal of the acoustical society of America. Cited in page 47

Bender(1974) Edward A Bender. The asymptotic number of non-negative integer matrices with given row and column sums. Discrete Mathematics, 10(2):217-223. Cited in page 42, 45 
Bethony et al.(2001) Jeffrey Bethony, Jeff T Williams, Laura Almasy, Rodrigo CorrêaOliveira, John C Blangero and Sarah Williams-Blangero. Genetic analysis of quantitative traits in highly ascertained samples: total serum ige in families with asthma. Genetic epidemiology, 21(S1):S174-S179. Cited in page 16

Bollen(1989) K. A. Bollen. Structural Equations with Latent Variables. Wiley. Cited in page 6

Bollen(1987) Kenneth A Bollen. Total, direct, and indirect effects in structural equation models. Sociological methodology, pages 37-69. Cited in page 17

Bollobás(1980) Béla Bollobás. A probabilistic proof of an asymptotic formula for the number of labelled regular graphs. European Journal of Combinatorics, 1(4):311-316. Cited in page 45

Bollobás(1998a) Béla Bollobás. Modern graph theory, volume 184. Springer Science \& Business Media. Cited in page 46

Bollobás(1998b) Béla Bollobás. Random graphs. In Modern graph theory, pages 215-252. Springer. Cited in page 44

Bonacich(1972) Phillip Bonacich. Factoring and weighting approaches to status scores and clique identification. Journal of Mathematical Sociology, 2(1):113-120. Cited in page 48

Bordenave(2008) Charles Bordenave. Eigenvalues of euclidean random matrices. Random Structures \& Algorithms, 33(4):515-532. Cited in page 3, 42, 44

Brouwer and Haemers(2011) Andries E Brouwer and Willem H Haemers. Spectra of graphs. Springer Science \& Business Media. Cited in page 43

Butler(2008) Steven Kay Butler. Eigenvalues and structures of graphs. ProQuest. Cited in page 43

Chen and Pearl(2014) Bryant Chen and Judea Pearl. Graphical tools for linear structural equation modeling. Technical report, Department of Computer Science, University of California, Los Angeles. Cited in page 6

Chickering(2002) David Maxwell Chickering. Learning equivalence classes of bayesiannetwork structures. Journal of machine learning research, 2(Feb):445-498. Cited in page 14

Colombo et al.(2012) Diego Colombo, Marloes H Maathuis, Markus Kalisch and Thomas S Richardson. Learning high-dimensional directed acyclic graphs with latent and selection variables. The Annals of Statistics, 40(1):294-321. Cited in page 15

Conover and Iman(1981) William J Conover and Ronald L Iman. Rank transformations as a bridge between parametric and nonparametric statistics. The American Statistician, 35(3):124-129. Cited in page 49, 50

Craddock et al.(2013) Cameron Craddock, Yassine Benhajali, Carlton Chu, Francois Chouinard, Alan Evans, András Jakab, Budhachandra S Khundrakpam, John D Lewis, Qingyang Li, Michael Milham et al. The neuro bureau preprocessing initiative: open sharing of preprocessed neuroimaging data and derivatives. Neuroinformatics. Cited in page 57 
Dahl et al.(2013) Andy Dahl, Victoria Hore, Valentina Iotchkova and Jonathan Marchini. Network inference in matrix-variate gaussian models with non-independent noise. arXiv:1312.1622. Cited in page 7

Daw et al.(2003) E Warwick Daw, John Morrison, Xiaojun Zhou and Duncan C Thomas. Genetic analysis workshop 13: simulated longitudinal data on families for a system of oligogenic traits. In BMC genetics, volume 4, page S3. BioMed Central. Cited in page 2, 8, 27

De Andrade et al.(2015) Mariza De Andrade, Debashree Ray, Alexandre C Pereira and Júlia P Soler. Global individual ancestry using principal components for family data. Human heredity, 80(1):1-11. Cited in page 2, 7, 32

de Oliveira et al.(2008) Camila M de Oliveira, Alexandre C Pereira, Mariza de Andrade, Júlia M Soler and José E Krieger. Heritability of cardiovascular risk factors in a brazilian population: Baependi heart study. BMC Medical Genetics, 9(1):32. Cited in page 2, 8, 32

Dempster(1972) Arthur P Dempster. Covariance selection. Biometrics, 28:157-175. Cited in page 6,14

Dempster(1969) Arthur Pentland Dempster. Elements of continuous multivariate analysis. Addison-Wesley Publ. Co. Cited in page 17

Dickey and Fuller(1979) David A Dickey and Wayne A Fuller. Distribution of the estimators for autoregressive time series with a unit root. Journal of the American statistical association, 74(366a):427-431. Cited in page 50

Dorogovtsev et al.(2003) Sergey N Dorogovtsev, Alexander V Goltsev, José FF Mendes and Alexander N Samukhin. Spectra of complex networks. Physical Review E, 68(4): 046109. Cited in page 3, 42,46

Duarte et al.(2014) Nubia E Duarte, Suely R Giolo, Alexandre C Pereira, Mariza De Andrade and Júlia P Soler. Using the theory of added-variable plot for linear mixed models to decompose genetic effects in family data. Statistical applications in genetics and molecular biology, 13(3):359-378. Cited in page 25

Erdôs and Rényi(1959) Paul Erdôs and Alfréd Rényi. On random graphs. Publicationes Mathematicae Debrecen, 6:290-297. Cited in page 42, 44

Erdos and Rényi(1960) Paul Erdos and Alfréd Rényi. On the evolution of random graphs. Publ. Math. Inst. Hung. Acad. Sci, 5(1):17-60. Cited in page 44

Evans(2002) David M Evans. The power of multivariate quantitative-trait loci linkage analysis is influenced by the correlation between variables. The American Journal of Human Genetics, 70(6):1599-1602. Cited in page 37

Freeman(1977) Linton C Freeman. A set of measures of centrality based on betweenness. Sociometry, pages 35-41. Cited in page 48

Friedman et al.(2008) Jerome Friedman, Trevor Hastie and Robert Tibshirani. Sparse inverse covariance estimation with the graphical lasso. Biostatistics, 9(3):432-441. Cited in page 7 
Friendly et al.(2013) Michael Friendly, Georges Monette and John Fox. Elliptical insights: Understanding statistical methods through elliptical geometry. Statist. Sci., 28(1):1-39. Cited in page 16

Fujita et al.(2017) André Fujita, Daniel Yasumasa Takahashi, Joana Bisol Balardin, Maciel Calebe Vidal and João Ricardo Sato. Correlation between graphs with an application to brain network analysis. Computational Statistics 85 Data Analysis, 109:76-92. Cited in page $3,42,49,67,73$

Füredi and Komlós(1981) Zoltán Füredi and János Komlós. The eigenvalues of random symmetric matrices. Combinatorica, 1(3):233-241. Cited in page 3, 42, 44

Gelman et al.(2013) Andrew Gelman, Hal S Stern, John B Carlin, David B Dunson, Aki Vehtari and Donald B Rubin. Bayesian data analysis. Chapman and Hall/CRC. Cited in page 42

Gilbert(1961) Edward N Gilbert. Random plane networks. Journal of the Society for Industrial and Applied Mathematics, 9(4):533-543. Cited in page 42, 44

Granger(1969) Clive WJ Granger. Investigating causal relations by econometric models and cross-spectral methods. Econometrica: Journal of the Econometric Society, pages 424-438. Cited in page 2, 48

Graybill(1976) FA Graybill. Theory and application of the linear model. wadsworth and brooks. Pacific Grove, CA. Cited in page 52

Grundy et al.(2005) Scott M Grundy, James I Cleeman, Stephen R Daniels, Karen A Donato, Robert H Eckel, Barry A Franklin, David J Gordon, Ronald M Krauss, Peter J Savage, Sidney C Smith Jr et al. Diagnosis and management of the metabolic syndrome: an american heart association/national heart, lung, and blood institute scientific statement. Circulation, 112(17):2735-2752. Cited in page 32

Hammersley and Clifford(1971) John M Hammersley and Peter Clifford. Markov fields on finite graphs and lattices. Unpublished manuscript. Cited in page 12

Hilden-Minton(1995) James Andrew Hilden-Minton. Multilevel diagnostics for mixed and hierarchical linear models. Doctoral Dissertation, UCLA. Cited in page 8, 21

Iman and Conover(1979) Ronald L Iman and William J Conover. The use of the rank transform in regression. Technometrics, 21(4):499-509. Cited in page 50

Kalisch et al.(2012) Markus Kalisch, Martin Mächler, Diego Colombo, Marloes H Maathuis and Peter Bühlmann. Causal inference using graphical models with the r package pcalg. Journal of Statistical Software, 47(11):1-26. Cited in page 24

Koller and Friedman(2009) Daphne Koller and Nir Friedman. Probabilistic graphical models: principles and techniques. MIT press. Cited in page 6, 9, 14

Lange(2003) Kenneth Lange. Mathematical and statistical methods for genetic analysis. Springer Science \& Business Media. Cited in page 2, 6, 18

Lauritzen(1996) Steffen L Lauritzen. Graphical models, volume 17. Oxford, United Kingdom: Clarendon Press. Cited in page 1, 6, 7, 12, 13 
Lauritzen and Spiegelhalter(1988) Steffen L Lauritzen and David J Spiegelhalter. Local computations with probabilities on graphical structures and their application to expert systems. J R Stat Soc Series B (Methodological), 50(2):157-224. Cited in page 6

Levy et al.(2000) Daniel Levy, Anita L DeStefano, Martin G Larson, Christopher J O'Donnell, Richard P Lifton, Haralambos Gavras, L Adrienne Cupples and Richard H Myers. Evidence for a gene influencing blood pressure on chromosome 17. Hypertension, 36:477-483. Cited in page 2, 8, 27

Lovász(1979) László Lovász. On the shannon capacity of a graph. IEEE Transactions on Information theory, 25(1):1-7. Cited in page 44

Lovász(1993) László Lovász. Random walks on graphs. Combinatorics, Paul erdos is eighty, 2:1-46. Cited in page 44

Loy and Hofmann(2015) Adam Loy and Heike Hofmann. Are you normal? the problem of confounded residual structures in hierarchical linear models. J. Comput. Graph. Stat., 24(4):1191-1209. Cited in page 8, 21

Lütkepohl(2005) Helmut Lütkepohl. New introduction to multiple time series analysis. Springer Science \& Business Media. Cited in page 49, 50, 52

Meinshausen et al.(2006) Nicolai Meinshausen, Peter Bühlmann et al. High-dimensional graphs and variable selection with the lasso. The annals of statistics, 34(3):1436-1462. Cited in page 13, 14

Oualkacha et al.(2012) Karim Oualkacha, Aurelie Labbe, Antonio Ciampi, Marc-Andre Roy and Michel Maziade. Principal components of heritability for high dimension quantitative traits and general pedigrees. Stat Appl Genet Mol Biol., 11(2). Cited in page 2, 7, 32, 38

Parlett(1998) Beresford N Parlett. The symmetric eigenvalue problem, volume 20. Society for Industrial and Applied Mathematics. Cited in page 22

Pearl(1988) Judea Pearl. Probabilistic inference in intelligent systems, 1988. Cited in page 6

Pearl(2000) Judea Pearl. Causality: models, reasoning, and inference. Cambridge University Press, Cambridge, UK. Cited in page 1, 6, 9

Pearl(2001) Judea Pearl. Direct and indirect effects. In Proceedings of the seventeenth conference on uncertainty in artificial intelligence, pages 411-420. Morgan Kaufmann Publishers Inc. Cited in page 17

Pearl and Verma(1991) Judea Pearl and T. S. Verma. A theory of inferred causation. $K R, 91: 441-452$. Cited in page 1, 15

Pearl and Verma(1990) Judea Pearl and T. S. Verma. Equivalence and synthesis of causal models. Proceedings of the 6th Conference on Uncertainty in Artificial Intelligence, pages 220-227. Cited in page 12

Power et al.(2012) Jonathan D Power, Kelly A Barnes, Abraham Z Snyder, Bradley L Schlaggar and Steven E Petersen. Spurious but systematic correlations in functional connectivity mri networks arise from subject motion. Neuroimage, 59(3):2142-2154. Cited in page 57 
Ribeiro et al.(2016) Adèle H. Ribeiro, Júlia M. P. Soler, Elias Chaibub Neto and André Fujita. Causal inference and structure learning of genotype-phenotype networks using genetic variation. In Big Data Analytics in Genomics. Springer, New York. doi: 10.1007/ 978-3-319-41279-5. URL http://www.springer.com/br/book/9783319412788. Cited in page $4,9,39$

Robinson(2009) William S Robinson. Ecological correlations and the behavior of individuals. International journal of epidemiology, 38(2):337-341. Cited in page 17

Rubin(1974) Donald B Rubin. Estimating causal effects of treatments in randomized and nonrandomized studies. Journal of educational Psychology, 66(5):688. Cited in page 6

Runge(1901) Carl Runge. Über empirische funktionen und die interpolation zwischen äquidistanten ordinaten. Zeitschrift für Mathematik und Physik, 46(224-243):20. Cited in page 57

Searle and Khuri(2017) Shayle R Searle and Andre I Khuri. Matrix algebra useful for statistics. John Wiley \& Sons. Cited in page 20

Siegmund and Langholz(2002) Kimberly D Siegmund and Bryan Langholz. Ascertainment bias in family-based case-control studies. American journal of epidemiology, 155(9): 875-880. Cited in page 16

Sjölander and Zetterqvist(2017) Arvid Sjölander and Johan Zetterqvist. Confounders, mediators, or colliders. Epidemiology, 28(4):540-547. Cited in page 16

Spirtes and Meek(1995) Peter Spirtes and Christopher Meek. Learning bayesian networks with discrete variables from data. In $K D D$, volume 1, pages 294-299. Cited in page 15

Spirtes et al.(2000) Peter Spirtes, Clark N Glymour and Richard Scheines. Causation, prediction, and search, volume 81 . MIT press. Cited in page 1, 9, 15

Talluri and Shete(2014) Rajesh Talluri and Sanjay Shete. Gaussian graphical models for phenotypes using pedigree data and exploratory analysis using networks with genetic and nongenetic factors based on genetic analysis workshop 18 data. In BMC proceedings, volume 8, page S99. BioMed Central. Cited in page 7

Team et al.(2013) R Core Team et al. R: A language and environment for statistical computing, 2013. Cited in page 54

Therneau(2012) T Therneau. Coxme: Mixed effects cox models, R package version 2.2-3, 2012. Cited in page 24

Töpner et al.(2017) Katrin Töpner, Guilherme JM Rosa, Daniel Gianola and Chris-Carolin Schön. Bayesian networks illustrate genomic and residual trait connections in maize (zea mays 1.). G3: Genes, Genomes, Genetics. Cited in page 7

Tsamardinos et al.(2006) Ioannis Tsamardinos, Laura E Brown and Constantin F Aliferis. The max-min hill-climbing bayesian network structure learning algorithm. Machine learning, 65(1):31-78. Cited in page 14

Tutte(1959) William T Tutte. On the symmetry of cubic graphs. Canad. J. Math, 11(4): 621-624. Cited in page 45 
Tzourio-Mazoyer et al.(2002) N. Tzourio-Mazoyer, B. Landeau, D. Papathanassiou, F. Crivello, O. Etard, N. Delcroix, B. Mazoyer and M. Joliot. Automated anatomical labeling of activations in spm using a macroscopic anatomical parcellation of the mni mri single-subject brain. NeuroImage, 15(1):273 - 289. Cited in page 58

Valente et al.(2010) Bruno D Valente, Guilherme JM Rosa, Gustavo de los Campos, Daniel Gianola and Martinho A Silva. Searching for recursive causal structures in multivariate quantitative genetics mixed models. Genetics, 185(2):633-644. Cited in page 7

Van Mieghem(2010) Piet Van Mieghem. Graph spectra for complex networks. Cambridge University Press. Cited in page 3, 42, 45

Von Wright(1971) Georg Henrik Von Wright. Explanation and understanding. Cornell University Press. Cited in page 1

Wang et al.(2003) Yang Wang, Deepayan Chakrabarti, Chenxi Wang and Christos Faloutsos. Epidemic spreading in real networks: An eigenvalue viewpoint. In 22nd International Symposium on Reliable Distributed Systems, 2003. Proceedings., pages 25-34. IEEE. Cited in page 44

Watts and Strogatz(1998) D.J. Watts and S.H. Strogatz. Collective dynamics of 'smallworld' networks. Nature, 393:440-442. Cited in page 42, 45

Whittaker(1990) Joe Whittaker. Graphical models in applied multivariate analysis. John Wiley 8 Sons. Cited in page 6, 13

Wiener(1956) Norbert Wiener. The theory of prediction modern mathematics for enginners. by Beckenbackh EF. New York, McGraw-Hill. Cited in page 2

Woodward(2005) James Woodward. Making things happen: A theory of causal explanation. Oxford university press. Cited in page 1

Wright(1921) Sewall Wright. Correlation and causation. Journal of agricultural research, 20(7):557-585. Cited in page 6

Ziogas(1996) A. Ziogas. Correction for ascertainment bias in family studies. Doctoral Dissertation, Department of Preventive Medicine, Biostatistics Division, University of Southern California. Cited in page 16 